\title{
Versicherungs-Statistik für 1916
}

\author{
Einleitung
}

D ie Versicherungs-Statistik für 1916 konnte gleich den drei vorhergehenden leider nur mi t erheblıcher Verspatung zur Veröff'ntlıchung gelangen. Nach Bescitigung der durch de $n$ Krieg hervorgerufenen Behinderung wird mit allem Nachdruck auf ein rechtzeitiges Er scheinen hingewirkt werden.

Unter Reichsaufsıcht stchen 1. diejenigen mlandischen privaten Versicherungsunterne hmungen, deren Geschaftshetrieb nicht durch die Satzung oder die sonstigen Geschäftsun terlagen auf das Gebict eines Bundesstaats beschränkt ist (\$2 des Versicherungsaufsichtsge setzes), soweit sie nicht bei sachlhch, urtlich oder hinsiclttlich des Personenkreises eng be grenzter Ausdelınung nach $\$ 3$ Abs. 2 a. a. $O$. der Aufsicht einer Landesbehörde überwi esen worden sind, 2. dicjenigen inleindischen privaten Unternehmungen, deren Gesc häftsbetrieb auf das Geliet eines Bundesstaats leschränkt ist, deren Beaufsichtigung ab er auf Grund von $\S 3$ Abs. 1 a. a. O. dem $\Lambda$ ufstchtsant ubhertragen ist, 3 . die auslän dischen Versicherungsunternehmungen, dıe im Inlande durch Vertreter, Bevollıächtigte, Ag enten oder sonstige Vermittler das Veısicherungsgeschatft betreiben (\$91 a a. 0.$)$, 4. in be schränhtem Umfange diejenigen mlindischen privaten Versicherungsunternelımungen, w elche die Rückversicherung in geset/hch aulsichtspflichtigen Versicherungszweigen zum Ge genstande haben (Bekanntmachung vom 18. Juni 1908, R.G.Bl. S. 40!)).

Ausgenommen von der Aufsicht waren im Berichtsjahre 1. Unternehmungen, welche di e Versicherung gegen Kursverluste ader die 'Transportversicherung betreiben (\$116 a. a. 0.), 2. die auf Grund landesrechtlicher Vorschriften errichteten Ililfskassen, die au $f$ Grund der Gewerbeordnung von Innungen oder Innungsverbänden errichteten Unterstũ tzungskassen sowie die auf Grund berggesetzlicher Vorschriften errichteten Knappsc haftskassen ( 122 a. a. 0.).

Grundsătzlich ausgeschlossen von unserer Statıstik sind von dell der Reichsaufsicht un terstehenden Unternelımungen diejenigen, deren Beaufsichtigung auf Grund des erwä lonten $\S 3$ Abs. 1 dem Aufsichtsamt überwiesen ist; es waren das im Jahre 1916 286 Bajerische, 655 Ilessische, 1 Necklenburg-Strelitzscher, 81 Schaumburg-Lippische un d 94 Lippische Versicherungsvereine, die ihren Geschäftsbetrieb über ihren Heimatssta at nicht hinaus erstrecken. Wenn ihre Zahl auch groß ist, so fallen sie ihrer Bede utung nach doch wenig ins Gewicht; das zeigen z. B. die Zusammenstellungen, die in den Geschäftsberichten des Aufsichtsamts seit dem Jahre I 905 regelmäßig gegeben 
sind. Ebenso sind ausgeschlossen die Krankenkassen, die infolge Aufhebung des Hilfskassengesetzes mit dem I. Juni I 9 I 2 unter Reichsaufsicht gekommen sind.

Fortgefallen sind wiederum auch die englischen und die französischen Gesellschaften, die in Fricdenszeiten in Deutschland arbciteten, weil sie sehr bald nach Kriegsausbruch neueVersicherungen in Deutschland nicht mehr abgeschlossen haben und ordnungsmäßige Rechnungsabschlüsse während des Krieges von ihnen nicht zu beschaffen waren.

Der in den Tafeln zusammengestellte Zahlenstoff berulit auf den Jahresabschlüssen, die dem Amte gemäß seinen *Vorschriften über die Rechnungslegung der vom Rcichsaufsichtsamte für Privatversicherung beaufsichtigten größeren Versicherungsunternehmungen " (Ver. A. f. P. I902 S. 23 ff.) und "Vorschiriften üler die Rechnungslegung der vom Reichsaufsichtsamte für Privatversicherung beaufsichtigten Rückversicherungsunternelımungen “ (Ver. A.f.P. I909 S. 2 ff.) eingereicht werden. Als Geschäftsjahr gilt bei der überwiegenden Mehrzahl der Unternehmungen das Kalenderjalır; wo das nicht der Fall ist, bezielıt sich diese Statistik auf dasjenige Geschäftsjalır, dessen Beginn in das Jahr 1916 fällt.

Die Nachprüfung der Abschlüsse für das Geschäftsjahr 1916 hat einige Zahılen der vorjählırigen Abschlüsse als unrichtig erwiesen, die bei der Bearbcitung der Statistik für I 9 I 5 nach den Berichten der Unternelımungen für richtig gehalten werden mußten. Diese Zahlen sind in den Anmerkungen zu den 'Tafeln für I 9 I 6 gekennzeichnet und außerdem unter den Berichtigungen am Schlusse dieses Bandes aufgeführt.

Die Anordnung des Stoffes ist dieselbe wie in den früheren Statistiken.

Die sämtlichen Unternelımungen sind nach der Art ihres Betriebs in fünf Gruppen eingeteilt, nämlich :

1. Lebensversicherung und Krankenversicherung,

2. Unfall- und Haftpflichtversicherung,

3. Viehversicherung, Hagelversicherung und sonstige landwirtschaftliche Versicherung,

4. Feuerversicherung (einschl. der Versicherung mittelbarer Schäden), Versicherung gegen Sturmschäden, Wasserschäden und Diebstahl,

5. Sonstige Versicherungszweige (darunter Glasversicherung und Rückversicherung).

Innerhalb dieser Gruppen sind die Unternehmungen nach den von ihnen betriebenen Versicherungszweigen getrennt; sie folgen einander aber in allen Tafeln in derselben Reihe und stets unter derselben Nummer.

Zur Veranschaulichung der Entwickelung der einzelnen Versicherungszweige in den letzten fünf Jahren ist der Statistik eine Tafel beigegeben. 


\section{Allgemeiner Überblick}

Diese Statistik berichtet eingehend über 253 deutsche und 34 ausländische Unter- Dı Unternehmungen. Von den deutschen Unternelımungen sind 120 Aktiengesellschaften; die übrigen beruhen, abgeselıen von wenigen Einzelunternelımern und Genossenschaften mit beschränkter Iaftpflicht, auf dem Grundsatze der Gegenseitigkeit.

Die Gegenseitigkeitsvereine beschränkten ihren Betrieb fast alle auf einen einzelnen Geschïftszweig; nur $16(16)^{*}$ ) von ihnen betätigten sich gleichzeitig in verschiedenen Versicherungszweigen. Alle arbeiteten vornehmlich unmittelbar; kein Verein betrieb lediglich Rückversicherung. Von den 120 Aktiengesellschaften waren 40 (35) ausschließlichc Rückversicherungsunternehmungen, sämtlich mit mehreren Zweigen; von den übrigen, auch unmittelbar arbeitenden 80 ( 80 ) Alitiengesellschaften pflegten 55 (55) mehrere Zweige. Die kleineren Geschäftszweige: die Sturmschäden-, die Wasserleitungsschäden-, die Glas-, die Kautions-, Bürgschafts-, Kiredit- sowie die Maschinenversicherung, aber auch die Unfall- und Haftpflichtversicherung werden fast ausschließlich von Aktiengesellschaften gepflegt. Die seit dem Jahre i 9 i 1 nachgewiesenen Versicherungen mittelbarer Schäden (Mictverlust-, Betriebsverlust- und Zuckerpreisdifferenzversicherung), die im Zusammenhange mit der Feuer- oder der Wasserleitungsschädenversicherung betrieben werden, sind nicht als besondere Geschäftszweige anzusehen.

Von den in dieser Statistik ausführlich behandelten unmittelbar versichernden deutschien Unternehmungen haben im Berichtsjahre gearbeitet in der Lebensversicherung 30 (30) Aktiengesellschaften und 32 (32) Gegenseitigkeitsvereine, in der Unfallversicherung 31 (30) Altiengesellschaften und 1 (1) Gegenseitigkeitsverein, in der IIaltptlichtversicherung 31 (30) Aktiengesellschaften und 1 (1) Verein, in der Viehversicherung 1 (1) Aktiengesellschaft und 26 (26) Gegenseitigkeitsvereine, in der Hagelversicherung 4 (4) Aktiengesellschaften (sämtlich mit Ausschluß anderer Versicherungsgeschäfte) und 12 (12) Gegenseitigkeitsvereine (von denen drei auch die Feuerversicherung und einer auch die Baulastversicherung betrieben), in der Feuerversicherung 40 (40) Aktiengesellschaften und 24 (24) Gegenseitigkeitsvereine, in der Versicherung gegen Einbruchdiebstahl 43 (43) Aktiengesellschaften und 13 (13) Gegenseitigkeitsvereine, und endlich in der Glasversicherung 21 (21) Aktiengesellschaften und 2 (2) Gegenseitigkeitsvereine. Bei 35 (37) Kaskoversicherungsunternehmungen waren am Schlusse des Berichitsjahrs über 7800 (8300) Fahrzeuge im Werte von 91,89 $(98,19)$ Millionen Mark versichert.

Auch die 40 (35) Rückversicherungsgesellschaften haben sich mit allen aufsichtspflichtigen Versicherungszweigen befaßt, und zwar (abgesehen von den weniger wichtigen Zweigen): 17 (16) mit der Lebensversicherung, 19 (17) mit der Unfallversicherung, 16 (16) mit der Haftpflichtversicherung, 7 (4) mit der Vieh- und 4 (4) mit der Hagelversicherung, 39 (34) mit der Feuerversicherung, 27 (26) mit der Einbruchdiebstahlversicherung und 8 (8) mit der Glasversicherung.

Die Zahl der in dieser Statistik belıandelten ausländischen Gesellschaften stellt sich wie im Vorjahre wiederum auf nur 34, weil die englischen und die französischen Unternelımungen fortgelassen sind. Von den berïcksichtigten hatten ihren Hauptsitz: 4 in den Vercinigten Staaten von Amerika, 1 in Belgien, 2 in Dänemark, 4 in den Niederlanden, 12 in Österreich, 2 in Schweden und 9 in der Schweiz. Gearbeitet haben 17 in der Lebensversicherung, 8 in der Unfall- und 6 in der Haftpflichtversicherung, 10 in der

*) Die engeklammerten Zahlen 11 diesem allgememen Uberblıche bezieheu sidı aut das vorige $J_{d} h$ 
Feuerversicherung, 13 in der Einbruchdiebstahlversicherung, 3 in der Glasversicherung, 2 in der Kautions- und Veruntreuungsversicherung, 4 in der Wasserleitungsschädenund 2 in der Maschinenversicherung.

Der Umfang des Versicherungsgeschäfts der auch unmittelbar arbcitenden deutschen Unternehmungen im Jahre 1916 wird durch die folgenden Ubersichten veranschaulicht, welche wegen der tiefgehenden Verschiedenheit der Grundlagen die Lebensversicherung einerseits und die übrigen Versicherungszweige anderseits getrennt behandeln.

\section{Umfang des Geschäfts der deutschen Versicherungsunternehmungen}

a) Lebensversicherung

\begin{tabular}{|c|c|c|c|c|c|c|c|c|}
\hline \multirow{2}{*}{ Versicherungsart } & \multirow{2}{*}{$\begin{array}{l}\text { Zahl der } \\
\text { Vetsuthe- } \\
\text { tungs- } \\
\text { scheme }\end{array}$} & \multirow{2}{*}{$\begin{array}{c}\text { Versiche- } \\
\text { rungs- } \\
\text { summen } \\
1 \text { non . }\end{array}$} & \multicolumn{4}{|c|}{$\begin{array}{l}\text { Zunahme (Alualime }- \text { ) } \\
\text { gegen das Vorjahr }\end{array}$} & \multicolumn{2}{|c|}{ 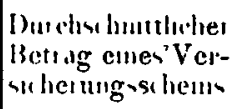 } \\
\hline & & & $\begin{array}{l}\text { Versiche- } \\
\text { rungs- } \\
\text { silieine }\end{array}$ & $\%$ & $\begin{array}{l}\text { Summen } \\
10 m 0 \text {, }\end{array}$ & $\%$ & $\begin{array}{c}\text { Ende } \\
1 ! 16 \\
M\end{array}$ & $\begin{array}{c}\text { Einde } \\
1915 \\
\mathscr{A}\end{array}$ \\
\hline Versicher ung anf den Todesfall & 2636 I98 & 13191513 & -44246 & $-1,65$ & -77563 & -058 & 5004 & 4950 \\
\hline Versiches ung auf den Lebensfall & 351344 & 605617 & -23911 & -637 & -34808 & $-54 t$ & I 724 & 1707 \\
\hline Rentens ersichen ung (Jahl'esi'enten) & 71382 & 30897 & $-\quad 1360$ & $|-1.87|$ & 267 & $-0,86$ & 433 & 428 \\
\hline Volhsversicheruig. & $7886+66$ & I 537197 & -28496 & -036 & 740 & 0,05 & 195 & 194 \\
\hline Zcitungsabonneutentetsu h & 1738234 & 116161 & 150862 & 950 & $13 \mathrm{JO}$ & 9.85 & 84 & $8+$ \\
\hline Sonstige heme Versicherung & 745193 & 409784 & -15251 & $|-2,01|$ & -3629 & $-0,88$ & 550 & 544 \\
\hline
\end{tabular}

Vergleicht man cliese Zilhlen mitdenen des Vorjahrs, so findet man bei der Veruicherung auf den Lebensfall, daß die Abnahme bes der Zahl der Versicherungsehrine wie bei den Versicherungssummen erheblich klemer geworden ist; der durchschnittheh anf cinen Schein entfallende Betrag ist fortdauernd, olwwohl langsam, gewachsen: von $1570 \mathscr{A l} \mathrm{im}$ Jahre I 902 auf $1724 \mathscr{M}$ im Berichtojahre. Die Ursache des fortdauemden Rickganges dieser Versicherungsart ist darin zu suchen, daß die Aussteucrversicherung jetzt vielfach durch die Todesfallversicherung mit Bonfikation im Erlehensfalle, die gemischte Versicherung und die Versicherung mit festem Auszahlungstermin. Versicherungsarten, die zu dor 'Todesfallversicherung gerechnet werden, ersetzt wird. - Der Zuwachs der Volksversicherung hat allmăhlich nachgelassen. Bis einschließlıch 1904 behef er sich bei den Scheinen und bei den Summen auf ungrfalhr 11 v II. des Bestandes zu Anfang des-Jahres; seitrlem ist er bis 1912 bei den Summen auf 6,19 v. II., bei den Zahlen der Scheine aul 4,10 v. H. gesunken; I913 schien stch wieder eine Ilebung einlesten zu wollen, als in I9I 4 der Krieg mit seinen zahılreichen Todesfallen einen Ruckschlag brachte, der sich auf 2,73 v H. bei den Summen und auf 1,51 v. II. bei den Sclícinen stellte und in 1915 auf 5,47 v. H. bei den Summen und auf 3,87 v. II. bei den Scheinen anstieg. Im Berichtsjahre bestand nur noch ein Rückgang von 0,36 v. H. bei den Scheinen, waihrend bei den Summen ein klciner Zugang von 0.05 v $H$. eingetreten ist. Die durchschnitthche Versicherungssumme hat zwischen 175 und $200 \mathscr{N}$ geschwankt. - Bei der großen Todesfallversicherung ist der infolge des Krieges eingetretene Ruckgang gegen das Vorjalır gesunken, nämlich von 2,94 v. II. auf 1,65 v. II. hei den Scheinen und ron 2,06 v. II. auf 0,58 v. H. bei den Summen Der durchschnittlich auf einen Schein kommende Betrag ist fortdauernd gewachisen: von $4533 \mathscr{H}$ zu linde 1903 auf $5004 \mathscr{H}$ am Inde des Berichtsjahrs - Beim Rentengeschäft hat der Zuwachs betragen ir IIundertsteln des Bestandes zu Anfang des Jahres

$\begin{array}{lllll}1912 & 1913 \quad 1914 & 1915 & 1916\end{array}$

bol der Zahl der Scheine $\quad$..

$-6,12 \quad-0,07$

$-1,14 \quad-2,20 \quad-1,87$

bet dem Jalu'sbetrage der icrsicherten Renten

$-3,91$

$\mathbf{1}, \mathbf{0 3}$

$078 \quad-2,23 \quad-0,86$ 
Im Berichtsjahre sind von den Versıcherten an Beiträgen und Ausfertigungsgebühren an die Unternchmungen gezdhlt worlen : in der Volksversicherung einschließlich der Zeitungsabonnentenversicherung 118,31 (118,i0) Mlillionen Mark und in der großen Versicherung einschlıeßlich der "sonstigen kleinen Versicherung “ 596,86 $(594,64)$ Mıllionen Mark; in demselhen Jahre sind für eingetretene Versicherungsfalle und für vor८eıtig aufgelöste Versicherungen in der Volksversicherung olıne die mit den Summen ausgezahlten Gewinnantelle 68,0t $(62,12)$ Mllhonen Mark und in der großen Versicherung 420,99 $(456,13)$ Millionen Mark bar an die Versicherten zurückgetlossen und außerdem 25,17 $(30,11)$ Millionen -NIark und 204,94 $(176,72)$ Mlllionen Mark, überwiegend aus den Beitragseinnalumen, den Primienreserven zugefuhrt worden. Die Zalulungen für Versicherungsfalle geben kcin rlchtıges Bıld fur die tatsächlıche Belastung, weil die Gesellschaften die Kriegstodesfalle verschieden behandeln. Einige zahlen sofort die volle Summe, andere sofort nur das Deckungskapital oder einen dariber hmausgehenden Teil der Versicherungssumme und machen cone Ergänzung nach dem Kriege von dem Stande shrer freien Nittel oder von einer Umlage abhängig. Einen befirıdigenden Uberblick uber die Lcistungen der Unternelımungen fur liriegstodesfalle wird man erst gewimen, wenn duese Verhaltnisse rollstandig abgewickelt sein werden.

Bei den anderen $Z_{w}$ rigen ist die Entwickelung auch in Friedenszeiten weniger gleichmäßig gewesen we her der Lebensversicherung. Immerhin hat die Unfallrersicherung seit Jahren dauernd an Umfing gewonnen, erst der Ki'leg hat in 1914 einen huckgang von 1,2 v II. in der Zahl der Versicherungen gehacht, in 1915 sogar einen solehen von 14,9 v. II. Im Berichtsjahre ist dagegen wieder em Zugang von 4,7 v. H. eingetreten. Die IIaftphlehtrersicherung ist auch 19 I 4 noch gewachsen, allerdungs nur noch um 2,6 v. II., wahrend sie in den besden vorhergehenden Jahren um 6,9 und 6,8 v. H. zugenommen latte. In 1915 aber war em Rückgang von $2,3 \mathrm{v}$. H. eingetreten, der sich im Berichtsjahre auf 1,0 v. II. ermaibigt hat.

Der Umfang der Viehversicherung, an den Summen gemessen, hatte 19i4 um $0,9 \mathrm{~V}$ H. des vorjahrigen Bestandes alıgenommen; I 9 I 5 war eme nicht viel gròßere Abnahme, namlich von 1,3 v. II., zu verzerchnen. Im Berıchtsjahre aber hat sich infolge der Wertstelgerung der Vichbestande eine Zunahme von 4j,3 v. II. crgeben. Die Ilagelversicherung unterliegt naturgemaß grỏßern Schwankungen. Im Vorjahre hatte die Zahl der Versicherungen um 1,9 v. II. und die Versicherungssumme um 11,5 v. II. zugenommen; jetzt hat der Zugang bei den Summen 10,6 v. H. des vorjährigen Bestandes betragen, wahrend die Anzahl der Scheine um 0,8 v. II. zurückgegangen ist.

Die bis zum Ausbruch des Krieges im großen und ganzen gleichmȧßige Fntwickelung des Geschafts der deutschen Feuerversicherungsuntemehmungen hatte in I 9 I 4 zum Teil dadurch eine Stergerung erfahren, daß infolge der llaftungs- und Uberführungsverträge, die zwischen den bei uns bis dahn tatıgen englischen und französischen Gesellschaften und deutschen Unternchmungen vereinbart wurden (vgl. Veroflentlichungen des A.f. P. 19I5 S. 7S), zahlresche beı ausländischen Gesellschaften laufende Versicherungen auf deutsche Giescllschalten übergingen Der Versicherungsbestand der deutschen Unternehmungen stellte sich Ende 1914 bei der Zahl der Versicherungen um 4,6 v. H. und bei der Versicherungssumme um 5,9 v. II. hoher als Ende 1913 und hatte damit ein wenig mehr zugenommen als in den vorhergehenden Fredensjahren. In 1915 stellte sich der Zugang nur noch auf 1,5 v. II. bei den Scheinen und auf 0,5 v. H. bei den 
Summen. Jetzt im Berichtsjahre ist nur noch ein Zugang bei den Summen, nämlich von 4,6 v. H., geblieben, wälırend die Scheine um 2,1 v. H. zurückgegangen sind.

Von dem gesamten bei den deutschen Lebensversicherungsgesellschaften Ende i 9 I 6 ( 9 I 5) versicherten Kapitale, $15890,27(15992,43)$ Millionen Mark, kommen 14328,53 $(14440,67)$ Millionen Mark auf das deutsche und 1561,74 $(1551,76)$ Millionen Mark auf das ausländische Geschäft; dagegen hatten die ausländischen Lebensversicherungsgesellschaften zu derselben Zeit im Deutschen Reiche $1039,72(1058,55)$ Millionen Mark versichert; ihr Anteil an dem gesamten deutschen Kapitalversicherungsgeschäfte, 15368,25 $(1$ j 499,22) Mlillionen Mark, stellt sich danach auf 6,8 $(6,8)$ v. H.

b) Schadenversicherung in den auch unmittelbar betriebenen Zweigen

\begin{tabular}{|c|c|c|c|c|c|c|c|c|c|}
\hline \multirow{3}{*}{ Versicherungszweig } & \multirow{3}{*}{$\begin{array}{l}\text { Zahll der } \\
\text { Versiche- } \\
\text { rungs- } \\
\text { scheme } \\
\text { (ummuttel- } \\
\text { lures } \\
\text { Geschäft) }\end{array}$} & \multicolumn{2}{|c|}{ Versicherungssummeı } & \multicolumn{4}{|c|}{ Zunahme gegen das Vorjahr } & \multirow{3}{*}{$\begin{array}{l}\text { Beiträge } \\
100 \mathscr{N}\end{array} \mid$} & \multirow{3}{*}{$\begin{array}{l}\text { Schädeı } \\
1 \mathrm{MMO} \mathscr{A}\end{array}$} \\
\hline & & \multirow{2}{*}{$\begin{array}{l}\text { unmittel- } \\
\text { bares } \\
\text { Geschäft } \\
1000 \mathscr{H}\end{array}$} & \multirow{2}{*}{$\begin{array}{c}\text { mittel- } \\
\text { bares } \\
\text { Geschäft } \\
1000 \mathscr{M}\end{array}$} & \multicolumn{2}{|c|}{$\begin{array}{l}\text { Versichesungs- } \\
\text { scheine }\end{array}$} & \multicolumn{2}{|c|}{$\begin{array}{l}\text { Versicher ungs- } \\
\text { summen }\end{array}$} & & \\
\hline & & & & Zahl & $\%$ & $1000 \mathscr{A H}$ & $\%$ & & \\
\hline Unfallversicher ung ..... & 1) 1273295 & & & (1) $\quad 57487$ & 4,7 & & . & 35307 & \\
\hline Il aftphichtversicherung . & 1) 2697665 & . & & 1) -27260 & $-\mathrm{I}, \mathrm{O}$ & & . & $48 \circ 55$ & 61 \\
\hline Vichiersicherung $\ldots \ldots$ & & $9688+2$ & II 685 & $?$ & $?$ & 305632 & 45,3 & 13976 & 1058.3 \\
\hline Ilıgels ersiclierung . . . . & 488840 & $\begin{array}{llll}3 & 874 & 26\end{array}$ & $13 \circ 378$ & $-\quad 4$ I26 & $-0,8$ & $38+940$ & 10,6 & $4556+$ & 33252 \\
\hline Feueiversicherung .. . & 12889702 & 153000604 & 17236797 & -272270 & $-2, \mathrm{I}$ & 7555222 & 4,6 & 318199 & 145980 \\
\hline Mieti ellustrel'sicherúng. & I 5973 & 96582 & 722 & 702 & $-4,2$ & $|-4333|$ & $-4,3$ & 129 & 16 \\
\hline $\begin{array}{l}\text { Betriebsver lustu eısiche- } \\
\text { ruing ............. }\end{array}$ & 2983 & 222943 & I 674 & 645 & 27,6 & 93025 & 70,7 & 1107 & 660 \\
\hline $\begin{array}{l}\text { Zucherpreisdifferenzver- } \\
\text { sicherung ...... . . } \\
\text { Sturmschädenversiche- }\end{array}$ & 53 & $255^{6}$ & 33 & 49 & $-48,0$ & -1370 & $-34,6$ & .5 & 一 \\
\hline $\begin{array}{c}1 \text { ung........ } \ldots \\
\text { Wasses leitumgsschanden- }\end{array}$ & 2739 & 224676 & 一 & 214 & 8,5 & 156 & $0, \mathbf{I}$ & 179 & 79 \\
\hline vetsicherung $\ldots \ldots \ldots$ & 136639 & 6418724 & 224845 & 60 & $-0,04$ & 250628 & 3.9 & 3304 & 1194 \\
\hline $\begin{array}{l}\text { Einbıuchdiebstahlver- } \\
\text { sicher ung. . . . . . . . }\end{array}$ & 1287095 & 17301785 & 4209842 & 25686 & 2,0 & 611023 & 2,9 & 17771 & 6727 \\
\hline Glasversichet ung ....... & 640720 & 368511 & 913 & -11662 & $-1,8$ & 6311 & 1,7 & $1033^{8}$ & 7031 \\
\hline $\begin{array}{l}\text { liautions- und Burg- } \\
\text { schaftsversuhes ung . . }\end{array}$ & 3372 & 90999 & I3I 425 & 496 & 17,2 & $44 I I 4$ & 24,7 & 1227 & 740 \\
\hline Kreditver stcherung..... & 173 & 3955 & 797 & 44 & $-20,3$ & 196 & 4,3 & 363 & 276 \\
\hline Maschnenverss herung & $229+$ & $50497^{\circ}$ & 19135 & 26 & $\mathrm{I}, \mathrm{I}$ & 108991 & 26,3 & 1074 & 855 \\
\hline Baulasti ersicherung .... & 240 & 7057 & - & $\mathbf{I}$ & 0,4 & 579 & 8,9 & 27 & 22 \\
\hline $\begin{array}{l}\text { Weitgegenständever- } \\
\text { sıchici ung } \ldots \ldots \ldots \ldots \\
\text { Veruntreungsvesiche- }\end{array}$ & 421 & 6807 & - & - & $-\mathbf{I}, 9$ & 412 & 6,4 & 26 & 9 \\
\hline $\begin{array}{l}\text { refuntreuungsvelsiche- } \\
\text { rung } \ldots \ldots \ldots \ldots \ldots\end{array}$ & 1009 & $26 \mathrm{II}_{4}$ & 2097 & 247 & 32.4 & 9363 & 49.7 & $24 \mathrm{I}$ & 48 \\
\hline Hypothehen ersicherung & $3^{I}$ & 9082 & - & 1 & $3 \cdot 3$ & 12 & 0,1 & $5^{1}$ & I 82 \\
\hline Stellenlosen el sicherung . & 2) 143029 & & & 6851 & 5,0 & & & 84 & 32 \\
\hline 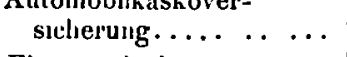 & $6 \mathrm{I}$ & 762 & 190 & 3 & 5,2 & 227 & $3^{I}, 3$ & 3 & I \\
\hline $\begin{array}{l}\text { Fliegerss hadenver- } \\
\left.\text { sleherung }{ }^{3}\right) \ldots \ldots\end{array}$ & 7892 & 732371 & $6 \pm 56$ & 5504 & 230,5 & 465574 & I 70,6 & 1446 & 1110 \\
\hline $\begin{array}{l}\text { 1) Einschl. des mit } \\
\text { 2) Mitglieder stilt } \\
\text { 3) AuB rdem } 1349 \\
\text { unmittelbares und mittellia } \\
\text { Die Beitıige und } \\
\text { sicherung sind diese Antei }\end{array}$ & $\begin{array}{l}\text { aren Ges } \\
\text { rsieheinug } \\
\mathscr{A} \text { Veis } \\
\text { Geseliaft } \\
\text { iden verst }\end{array}$ & $\begin{array}{l}\text { ifts. } \\
\text { cheine. } \\
\text { herumgssur } \\
\text { ucht bekas } \\
\text { ien sich e }\end{array}$ & $\begin{array}{l}\text { ne, zu v } \\
\text { t ist } \\
\text { chll. der }\end{array}$ & hend & ah & Scheine & sowi & d & nnung in \\
\hline
\end{tabular}


c) Versicherung in ausschließlich mittelbar betriebenen Zweigen

\begin{tabular}{|c|c|c|}
\hline Vorsicherungszweig & $\begin{array}{l}\text { Beitunge } \\
\text { tür engene } \\
\text { Rechumung } \\
1000 \text { Al }\end{array}$ & $\begin{array}{l}\text { Schäden } \\
\text { lür eigene } \\
\text { Rechunug } \\
1000 \mathscr{A}\end{array}$ \\
\hline Leluensversicherung ....... & 53277 & 20429 \\
\hline Unfall- und Ilaftpllichtversicherung & 18678 & 8006 \\
\hline Ilagelversicherung $\ldots \ldots \ldots \ldots$ & 6402 & 6532 \\
\hline Feuerversicherung ............ & 154568 & 80024 \\
\hline Sonstige aufsichtsplichtige 7wrige. & 12120 & 4271 \\
\hline Zusammen .... & 245045 & 119262 \\
\hline
\end{tabular}

Beurteilt man in der Unfall- und Haftpflichtversicherung den Umfang des Geschäfts nach der Jahresbeitragseinnalıme, so kommen von dem mit $83, \mathbf{3 6}(85,56)$ Millionen Mark zu bewertenden gesamten Geschäfte der deutschen Gesellschaften 75,79 (78,37) Millionen Mark auf das deutsche und $7,57(7,19)$ Millionen Mark auf das ausländische Geschäft; die ausländischen Gesellschaften haben aus ihrem deutschen Geschäfte 13,28 $(12,96)$ Millionen Mark eingenommen; sie sind also an dem gesamten deutschen Geschäfte mit $14,9(14,2)$ v. H. beteiligt gewesen.

Im unmittelbaren Feuerversicherungsgeschäfte hatten die großen deutschen Versicherungsgesellschaften insgesamt 153001 (147540) Millionen Mark versichert; davon kamen 14459 Ј (139013) Millionen auf das deutsche und 8406 (8527) Millionen Mark auf das auslindische Geschäft. Die ausländischen Unternehmungen einschlicßlich der hier sonst nicht weiter berücksichtigten Unternehmungen mit weniger als $100000 \mathscr{N}$ Beitragseinnahme aus dem deutschen Geschäfte hatten im unmittelbaren deutschen Geschäft um dieselbe Zeit 5803 (5740) Millionen Mark übernommen. Von diesen Zalilen sind indessen die auf das ausländische Geschäft der deutschen Gesellschaften und die auf das deutsche Geschäft der ausländischen Gesellschaften bezüglichen unsicher; sie können nur als untere Grenze angesehen werden. Die deutschen Unternelimungen konnten keine vollständigen Angaben machen, weil ihnen der Verkehr mit ihren ausländischen Vertretungen infolge von Kriegsmaßnalımen erschwert oder unmöglich gemacht war und das Geschäft der Gesellschaften mit dem Sitze im feindlichen Auslande fehlt, soweit es nicht infolge der erwälınten Uberführungsverträge auf deutsche Gesellschaften übergegangen und bei diesen nachgewiesen ist. Rechnet man unter diesem Vorbehalte zu dem deutschen Geschäfte noch die Summen, welche nach den "Mitteilungen für die öflentlichen Feuerversicherungsanstalten " die im Deutsclıen Reiche bestehenden 48 öfentlichen Anstalten versichert hatten, nämlich 93217 (90 8!16) Millionen Mark, so hat man in 243615 (235 649) Millionen Mark sehr nahe den Mindestwert der gegen Feuersgefahr versicherten deutschen Objekte, an dem übrigens auch noch die bei den kleinen Vereinen und den unter Landesaufsicht stelienden privaten Gegenseitigkeitsvercinen versicherten Werte fehlen, die insgesamt jedoch nur wenige Hundertteile der socben angegebenen Summe ausmachen werden. - Von obiger Summe kamen auf die deutschen privaten Unternehmungen unter Reichsaufsicht $59,3(59,0)$ v. H., auf die ausländischen Gesellschaften $2,4(2,4)$ v. H. und auf die deutschen öffentlichen Anstalten $38,3(38,6)$ v. H.

Aus dem unmittelbaren deutschen Geschäfte sind von den obigen privaten Unternehmungen und von den öffentlichen Feuerversicherungsanstalten im Jahre 1916 für 
Lebensversicherung $691,33 \quad(692,06)$, für Unfall- und Haftpflichtversicherung 85,82 $(87,59)$ und für Feuerversicherung $357,62 \quad(338,89)$, insgesamt $1134,77 \quad(1118,54)$ Millionen Mark an Beiträgen vereinnalımt worden, das sind rund $3,2(3,1)$ Millionen Mark auf den 'Tag.

Die folgenden Ubersichten sollen einen Einblick in die Betriebsergebnisse des Berichtsjalırs und in den Vermögensstand am Schlusse des Geschaiftsjahrs für die Gesamtheit der deutschen privaten Unternelımungen mit Ausnahme der Kaskoversicherungsvereine gewälıren.

Hierzu ist folgendes zu bemerken: Es ist schon erwähnt, daß die meisten Aktiengesellschaften mehrere Versicherungszweige betreiben. Die Gesellschaften dieser Art geben dann hinsichtlich ihres Lebensversicherungsgeschärts für die Volksversicherung einerseits und für die gewóhnliche Versicherung emschlıeßlıch der "sonstigen kleinen Versicherung" anderseits besondere Gewinn- und Verlustrechnungen, aus denen die folgenden Zahlen abgeleitet worden sind.

\section{Hauptsächlichste Einnahme- und Ausgabeposten der Lebensversicherung}

1 ก00 $\mathscr{H}$

\begin{tabular}{|c|c|c|c|c|c|c|c|c|}
\hline$B$ etrlebsat & $\begin{array}{c}\text { Beıtuage } \\
\text { und } \\
\text { Ausleıtı- } \\
\text { gunirs- } \\
\text { gebulien }\end{array}$ & \begin{tabular}{|c|} 
Ver- \\
gutumgen \\
der \\
Ruchver- \\
sicherer
\end{tabular} & $\begin{array}{c}\text { Kapital- } \\
\text { estluge } \\
\text { (tein) }\end{array}$ & $\begin{array}{c}\text { Yahlinugen! } \\
\text { fur } \\
\text { Versiche- } \\
\text { iungratalle }\end{array}$ & $\begin{array}{l}\text { Rucli- } \\
\text { sesolic- } \\
\text { rungs- } \\
\text { beitinge }\end{array}$ & $\mid \begin{array}{c}\text { Zu- } \\
\text { lulı ungen } \\
\text { 7u den } \\
\text { Ru(hligen } \\
\text { (renu) }\end{array}$ & $\begin{array}{c}\text { Ver- } \\
\text { waltungs- } \\
\text { hosten und } \\
\text { Sieuern }\end{array}$ & $\begin{array}{c}\text { Jahres- } \\
\text { uber- } \\
\text { schuB }\end{array}$ \\
\hline Volksi eisicherung ) & II 8387 & I 674 & 33212 & $68 \times 10$ & I 643 & 30778 & 28272 & 2 I 355 \\
\hline $\begin{array}{l}\text { Große Versweher ung und "son- } \\
\text { stige hleme Veisil herung " }\end{array}$ & 588906 & $29756 !$ & 240480 & 424793 & 27645 & I9I 820 & 52651 & 159005 \\
\hline Berufsveremgungen & 16529 & 274 & 5560 & 5871 & 249 & $\mathrm{r} 3658$ & 695 & I 747 \\
\hline Zusammen & 723822 & 31704 & 279261 & 498774 & 29537 & 236256 & 81618 & 182107 \\
\hline
\end{tabular}

Bei den übrigen Versicherungszweigen verfahren die Gesellschaften in ihren Rechnungsabschlussen verschieden; sie trennen entweder alle Einnalıme- und Ausgabeposten nach Geschäftszweigen oder nur einen 'Teil dieser Posten, während sie die übrigen, besonders die Kapitalerträge und die allgemeinen Verwaltungskosten, nur in einer Summe angeben. In den fur das Auf́sichtsaint bestimmten Aufstellungen werden indessen auch die allgemeinen Posten auf die einzelnen $\mathrm{Zweige}$ verteilt; daher kann in dieser Statistik für jeden Zweig der Gewinn aus dem eigentlichen Versicherungsbetrieb angegeben werden. Nur die Unfall- und Haftpflichtversicherung macht eine Ausnahme, weil hier von den Kapitalerträgen ein Tell dem Versicherungsbetriebe zugerechnet werden muß und dieser Teil nicht ausgeschieden werden kann. Aus den be $u$ üglichen 'Tafeln ist die folgende Übersicht zusammengestellt worden. Darin sind als Sonstige Betriebs-Einnahmen die Ersparnisse aus der Schadenrücklage sowie die Abnahme der Prämienreserven und Uberträge und bei der Viehversicherung der Erlös aus verwertetem Vıeh, als Sonstige Betricbs-Ausgaben etwaige Zuschüsse zur Schadenrücklage und die Zunahme der Pramienreserven und Übertrage, bei der Feuerversicherung auch die Leistungen zu gemeimützigen $\mathrm{Zwecken} \mathrm{aufgefülrt.} \mathrm{Die} \mathrm{Anteile} \mathrm{der} \mathrm{Rück-}$ 
versicherer sind ausgeschlossen. Die Schadenzahlungen enthalten auch die Schadenermittelungskosten.

Die Rückversicherungsgesellschaften haben dem Aufsichtsamt über ihre Geschäftsführung in einer Form zu berichten, die eine Frmittelung des Gewinns aus dem reinen Versicherungsbetriebe weder für einzelne Zweige noch für das gesamte Geschäft gestattet; in die nachstehende Tafel sind daher als Sonstige Einnahmen und Ausgaben die Summen der Jahreseinnahmen (ohne Gewinnvortrag) und Jahresausgaben nach Abzug der Beiträge einerseits und der Schäden nebst Verwaltungskosten und Steuern anderseits aus der Gewinn- und Verlustrechnung eingestellt.

\section{Aus der Betriebsrechnung der deutschen Schaden- und Rückversicherungsunternehmungen für 1916}

\begin{tabular}{|c|c|c|c|c|c|c|}
\hline Versicherungszwe 1 g & $\begin{array}{l}\text { Betriebs-Eim } \\
\text { Beitrage } \\
\text { und Neben- } \\
\text { leistungen } \\
\text { der Ver- } \\
\text { sicherten }\end{array}$ & Sonstige & $\begin{array}{l}\text { Betriebs-Au } \\
\text { Schaden- } \\
\text { zahlungen }\end{array}$ & $\begin{array}{l}\text { sgaben } \\
\text { Sonstige }\end{array}$ & $\begin{array}{c}\text { Ver- } \\
\text { waltungs- } \\
\text { kosten } \\
\text { und } \\
\text { Steuern }\end{array}$ & $\begin{array}{l}\text { UberschuB } \\
\text { (Verlust -) } \\
\text { des Ver- } \\
\text { sıcherungs- } \\
\text { betrielss }\end{array}$ \\
\hline Unfall- und Haftpflichtversiches ung . & 82846 & & 49014 & & 30381 & \\
\hline Viehversichetung & 12244 & 2069 & IOOOI & -. & 2928 & 1384 \\
\hline Hagelv ersicherung . .... . & 48418 & - & 34501 & - & 6973 & 6944 \\
\hline $\begin{array}{l}\text { Feuerv ersicherung (einschl.Vers mittelb } \\
\text { Schaden) }\end{array}$ & 183371 & 14374 & 79512 & 12460 & 56995 & $4877^{8}$ \\
\hline Sturmschadenversicherung , . & 38 & 5 & 22 & $\mathbf{I}$ & 17 & 3 \\
\hline Wasser leitungsschadenversicherung & $2827^{\prime}$ & 249 & 1144 & 70 & 1027 & 835 \\
\hline Eınbruchdıebstahlversicherung & I 2294 & ז 949 & 6273 & 841 & $435^{8}$ & 2771 \\
\hline Gllasversicherung & 9613 & 296 & 6306 & 390 & 3068 & 145 \\
\hline Kautions- und Burgschafisversicherung & 568 & 446 & 631 & 128 & 185 & 70 \\
\hline Kreditversicherung $\quad$.. & $158 !$ & 37 & 87 & I9 & 50 & 39 \\
\hline Maschmenversicherung . . & 307 & 25 & 246 & 12 & $8 \mathrm{I}$ & - \\
\hline Baulastversıcherung $\quad$. & 27 & - & 22 & I & 2 & 2 \\
\hline Wertgegenstāndeversıcherung . & 13 & 3 & 6 & $\ldots$ & 6 & 4 \\
\hline Veruntreuungsversicherung . . & 96 & 1 & 34 & 25 & 42 & -- \\
\hline Hypothekenversicherung .. & 211 & 16 & $19^{\prime}$ & 20 & 3 & - \\
\hline Stellenlosenversicherung & 84 & - & $3 x^{1}$ & - & 45 & 8 \\
\hline Automobilkaskoversicherung . & 1 & 8 & $\mathbf{I}$ & - & I & 7 \\
\hline Fliegerschadenversicherung & $5^{80}$ & 60 & $47 \mathrm{I}$ & 29 & I 24 & 16 \\
\hline Rückversıcherung & 324320 & 27490 & 169218 & 75775 & 92245 & 11572 \\
\hline
\end{tabular}

Eine Bilanz wird immer nur für das Gesamtgeschäft aufgestellt und bei derjenigen úruppe nachgewiesen, zu der die Gesellschaft ihrem aufsichtspflichtigen Hauptbetriebe nach zu rechnen ist. Die Bilanz umfaßt also gegebenenfalls auch das Transportversicherungsgeschäft, über das die Statistik im übrigen nur bei den Rückversicherungsgesellschaften einige Angaben bringt, weil die Transportversicherung der Beaufsichtigung nicht unterliegt. In den Gewinn der Bilanz ist auch der Gewinnvortrag aus dem Vorjahr eingeschlossen.

In der folgenden Utbersicht sind berücksichtigt 120 Aktiengesellschaften mit 680 , 39 Millionen Mark Aktienkapital, wovon 178,33 Millionen Mark eingezahlt sind, und 92 Gregenseitigkeitsvereine mit einem Garantiekapitale von 2,97 Millionen mit 1,60 Millionen 
Einzahlung. Die für die Aktiengesellschaften in $§ 262$ des Handelsgesetzbuchs und für die Gegenseitiglieitsvereine in $\$ 37$ des Versicherungsaufsichtsgesetæes vorgeschriebenen gesetzlichıen Rücklagen beliefen sich Ende 19 i 6 für alle Unternelımungen zusammen auf 204,47 Milhonen; dazu kamen 390,93 Millionen Mark Sonderrücklagen, die freilich zum Teil nicht als freie Rücklagen betrachtet werden können. Die Prämienreserven und die Prämienüberträge, 6071,92 Hillionen, meist auch die Gewinnrücklagen, 612,82 Millionen, sowie die Schadenrïcklagen, 418,95 Millionen, sind als gebunden anzusehen, da sie als rechnungsmäßige Deckung für die teils bereits entstandenen, teils roraussichtlich in Zukunft noch entstehenden Ansprüche der Versicherten aus geleisteten Beitragszahlungen dienen.

\section{Aus der Bilanz der deutschen Unternehmungen für Ende 1916 - Gesamtgeschäft}

$1000 \mathscr{M}$

\begin{tabular}{|c|c|c|c|c|c|c|c|}
\hline \multirow[b]{2}{*}{$\begin{array}{c}\text { Posten der Vermogensuerte } \\
\text { und Veabindlichlienten }\end{array}$} & \multicolumn{7}{|c|}{ If a uptzweig } \\
\hline & $\begin{array}{l}\text { Lebens- } \\
\text { r ersiche- } \\
\text { rung }\end{array}$ & $\begin{array}{c}\text { Unfall- } \\
\text { und Haft- } \\
\text { pflicht- } \\
\text { versiche- } \\
\text { rung }\end{array}$ & $\begin{array}{c}\text { Vieh- } \\
\text { versiche- } \\
\text { rung }\end{array}$ & $\begin{array}{c}\text { Hagel- } \\
\text { versiche- } \\
\text { rung }\end{array}$ & $\begin{array}{c}\text { Feuer- } \\
\text { versiche- } \\
\text { rung }\end{array}$ & $\begin{array}{c}\text { Glas- } \\
\text { versiche- } \\
\text { rung }\end{array}$ & $\begin{array}{l}\text { Ruick- } \\
\text { versiche- } \\
\text { rung }\end{array}$ \\
\hline Vermögenswerte insgesamt . . & 7035590 & 416704 & 11682 & 66414 & 893837 & 22314 & 714417 \\
\hline $\begin{array}{c}\text { Verpflılitungen der } \Lambda \text { ktıonale oder } \\
\text { Galanten .. }\end{array}$ & 120515 & 46152 & $75^{\circ}$ & 18117 & 1838 I5 & 4363 & I 29726 \\
\hline Kapitalanlagen & 6530297 & 295884 & 7073 & 38838 & $55^{2} \quad 185$ & I 3468 & 288061 \\
\hline Forderungen & 314171 & 71258 & $355^{\circ}$ & 9295 & 152999 & 4203 & 248763 \\
\hline Kissenbestand & 5989 & $3^{87}$ & 214 & 160 & 930 & $5 \mathrm{I}$ & 220 \\
\hline Uhrige Vermogenswerte ... & 64618 & 3023 & 95 & 4 & 3908 & 229 & 47647 \\
\hline Veslust . & - & - & - & - & - & - & - \\
\hline Verbindlichkeiten insgesamt & 7035590 & 416704 & 11682 & 66414 & $8: 3837$ & 22314 & 714417 \\
\hline Ahtien- oder Garantichapital & 163474 & 62000 & I 000 & 25529 & 249374 & 6312 & 175674 \\
\hline Genetzlirhe Rucluage. & 33815 & 34057 & 4886 & 15541 & 73530 & I 726 & 40918 \\
\hline Sondeı ruchlagen & 216602 & $38: 97$ & 606 & 15556 & 82063 & I 232 & 36669 \\
\hline Gewımı ucklagen & 600924 & $1 \mathrm{I} 89 \mathrm{I}$ & - & - & - & - & - \\
\hline $\begin{array}{c}\text { Guthahen von Versicherungsunter- } \\
\text { nehmungen. }\end{array}$ & 18970 & 23250 & I 16 & 108 & 64022 & 675 & $863^{89}$ \\
\hline Pramenteserven u Prámınübertıäge & 5526882 & 130785 & 2386 & I & 221828 & 5668 & 184373 \\
\hline Schadenrur hlagen . $\ldots . . \quad$. & 89904 & 78405 & 617 & 800 & 86736 & 4359 & I 58 I 28 \\
\hline Ubrige Veıbundlichibesten & 203639 & 15985 & I 454 & 3580 & 54716 & 1472 & I 1943 \\
\hline $\begin{array}{lllllllll}\text { Gewinn } & \ldots & \ldots & \text {. }\end{array}$ & 181380 & 22134 & 617 & 5299 & 6 I 568 & 870 & 20323 \\
\hline
\end{tabular}

Die Kapitalanlagen aller dieser 212 Unternehmungen haben Ende 1916 insgesamt 7725,81 Millionen Mark betragen; davon entfallen 186,73 Millionen auf Grundbesitz, 5303,96 Millionen auf IIypotheken und Grundschulden, 456,45 Millionen auf Darlehen an öffentliche Korperschaften, 8,03 Millionen auf Darlehen auf Wertpapiere. 1 212,61 Mıllionen auf Wertpapiere, 53\%,46 Millionen auf Darlehen auf Versicherungen, 13,70 Millionen auf Wechsel und 4,87 Millionen auf sonstige Anlagen 
Die Verteilung auf diese Wertarten ist aber bei den verschiedenen Versicherungszweigen und zum 'Teil auch bei den beiden Geschäftsformen eine recht verschiedene; auch wird sie durch heimische Übung und Gesetzgebung mannigfach beeinflußt. Näheres hierüber findet man in der "Entwıckelung " $S$ 62*).

\section{Kapitalanlagen Ende 1916 (Bilanzwert)}

\begin{tabular}{|c|c|c|c|c|c|c|c|c|c|}
\hline \multirow{3}{*}{$\begin{array}{c}\text { Versicherungszweig } \\
\text { und } \\
\text { Gesellschaftoform }\end{array}$} & \multirow[b]{3}{*}{$\begin{array}{l}\text { Insgesant } \\
1000 \mathrm{M}\end{array}$} & \multicolumn{8}{|c|}{ Von $1000 \mathscr{H}$ der Kapitalanlagen kommen auf } \\
\hline & & \multirow[b]{2}{*}{$\begin{array}{c}\text { Grund- } \\
\text { besitz } \\
M\end{array}$} & \multirow[b]{2}{*}{$\begin{array}{c}\text { Hypo- } \\
\text { theken } \\
M\end{array}$} & \multirow{2}{*}{$\begin{array}{c}\text { Darlehen } \\
\text { an offent- } \\
\text { liche } \\
\text { Korper- } \\
\text { schaften } \\
\mathscr{A}\end{array}$} & \multirow[b]{2}{*}{$\begin{array}{c}\text { Wert- } \\
\text { papuere }\end{array}$} & \multicolumn{2}{|c|}{ Darlehen auf } & \multirow[b]{2}{*}{$\begin{array}{c}\text { Wech- } \\
\text { sel }\end{array}$} & \multirow[b]{2}{*}{$\begin{array}{l}\text { Sonstige } \\
\text { Anlagen }\end{array}$} \\
\hline & & & & & & $\begin{array}{c}\text { Wert- } \\
\text { papiere } \\
\varkappa\end{array}$ & \begin{tabular}{|c|} 
Ver- \\
sithe- \\
rungen \\
$\mathscr{M}$
\end{tabular} & & \\
\hline \multicolumn{10}{|c|}{ a) bei den deutschen Unternehmungen } \\
\hline Lebenst (Aktiengesellschaften. . & $|3998821|$ & $23 i$ & 758 & 51 & 89 & $(\mathrm{o}, \mathrm{I} \mathrm{I})$ & 79 & $(0,0006)$ & - \\
\hline versiche- $\{$ Gegenseltigkeitsvereme & 2380199 & 8 & 729 & 78 & 94 & I & 89 & $\mathbf{I}$ & - \\
\hline rung Belufsveremigurgen & 151277 & $8 !$ & 623 & 76 & 276 & $(0,016)$ & 17 & - & - \\
\hline $\begin{array}{l}\text { Unfall- und IIaftyflichti ersicherungs- } \\
\text { gesellschidten }\end{array}$ & 295884 & 80 & 477 & I 49 & $277 j$ & & 14 & $\left(03^{2}\right)$ & - \\
\hline Viehver- ( Ahtiengesellschaften & 629 & 172 & - & - & 828 & $-\quad$ & $-\quad$ & - & -- \\
\hline sicher ung Gegenseitıgheitsverene & 6444 & 35 & 148 & $(0.24)$ & 817 & - & - & 一 & $(0,008)$ \\
\hline Hagelver- $\{$ Ahtiengesellschatten & 24489 & 18 & 68 & - & 873 & 8 & - & 33 & - \\
\hline sicher ung ( Gegenscitıghertsver eme & 14349 & 30 & 106 & I & 812 & $(0,21)$ & - & 49 & 2 \\
\hline Feuerver- $\{$ Aktiengesellschaften & 445941 & 74 & 433 & 2 & 452 & $10 !$ & 14 & II & 4 \\
\hline sicherung $\{$ Gegenseltigkeitsvereme & 106245 & $27 !$ & 339 & 99 & 521 & $-\quad i$ & $-\quad 1$ & 4 & 10 \\
\hline Glasver- (Aktiengesellschaften & 12483 & 112 & 687 & $\perp$ & 200 & I & - & - & - \\
\hline sicherung Gegenseitigheitsvereine & 985 & 35 & 699 & - & 266 & $-\quad$ & - & - & - \\
\hline Ruckversicherung Aktiengesellschaften & 288 o6 I & 37 & 204 & 5 & 730 & 1 & - & 16 & 7 \\
\hline Insgesamt & 7725807 & 24 & 686 & $\mathbf{5 9} !$ & 157 & 1 & 70 & 2 & 1 \\
\hline \multicolumn{10}{|c|}{ b) bei den ausländischen Unternehmungen } \\
\hline bei 4 amerikamschen Gesellschaften & $\mid 8505$ I $38 \mid$ & 29 & 205 & $\mathbf{I}$ & $5^{88}$ & $(0,06)$ & $177 !$ & - & - \\
\hline " 1 belgischen Gesellschaft & 4329 & 59 & $-i$ & -- & 829 & 112 & -1 & - & - \\
\hline " 2 dänıschen Gesellschaften & 79124 & 42 & 210 & - & 668 & - & 80 & - & - \\
\hline " 4 mederlāndischen Gesellschaften & 173667 & 114 & 263 & - & 520 & 18 & 83 & - & 2 \\
\hline " 12 österreichıschen Gesellschaften & I 049022 & 91 & 301 & 56 & 452 & $9:$ & 90 & I & - \\
\hline " 2 schwedischen Gesellschaften. & 98935 & 40 & 469 & $45^{\prime}$ & 332 & $44^{\prime}$ & 70 & 一 & - \\
\hline - 9 schwelzerischen Gesellschaften & $43^{8} 06 \mathrm{I}$ & 62 & 573 & I4। & 308 & I & 40 & 2 & - \\
\hline
\end{tabular}

Über die Zusammensetzung der unter den Kapitalanlagen aufgefuhrten Wertpapierbestände soll die folgende Zusammenstellung Aufschluß geben. Zu bemerken ist dazu nur, daß der Verteilung der Nennwert zugrunde gelegt worden ist und daß der bei den einzelnen Zweigen eingestellte Nennwert des Gesamtbestandes wegen besonderer Umstände, die aus den Fußnoten zu den betreffenden Tafeln ersichtlich sind und hier nicht angeführt zu werden brauchen, nicht überall genau dem bei den Kapitalanlagen in der Bilanz verrechneten Werte entspricht; die Abweichungen sind übrigens so gering, daß sie hier nicht ins Gewicht fallen.

*) Die Entwickelung des priaten Versicherungswesens unter Reichsaufsıcht in dem Jahrfunft 1907 bis 1911 Herausgegeben vom Reichsaufsıchtsante fur Pri atr ersıcherung, Berlm 1913, J Guttentag, Verlagsbuchhandlung G m b H - Diese Schrift ist un dem vorliegenden Buche kurz als "Entwickelung“ angeführt 
Verteilung der Wertpapiere (Nennwert)

\begin{tabular}{|c|c|c|c|c|c|c|c|c|c|}
\hline \multirow{3}{*}{$\begin{array}{l}\text { Versicherungszweig } \\
\text { und } \\
\text { Gesellschaftsform }\end{array}$} & \multirow{3}{*}{$\begin{array}{c}\text { Wert- } \\
\text { papiere } \\
\text { ins- } \\
\text { gesamt } \\
1000 \mathscr{A}\end{array}$} & \multicolumn{8}{|c|}{ Von $1000 \mathscr{M} \mathrm{der}$ Wertpapiere kommen auf } \\
\hline & & \multicolumn{5}{|c|}{ Inländısche Wertpapiere } & \multicolumn{3}{|c|}{ ausland ische Wertpapiere } \\
\hline & & $\begin{array}{c}\text { Staats- } \\
\text { anleihen } \\
\text { und } \\
\text { staatich } \\
\text { gedechte } \\
\text { Anleshen } \\
\mathscr{M}\end{array}$ & $\begin{array}{c}\text { Kom- } \\
\text { munal- } \\
\text { anleihen } \\
\mathscr{A}\end{array}$ & $\begin{array}{c}\text { Pfand- } \\
\text { briefe } \\
\text { und } \\
\text { Konmu- } \\
\text { nalobli- } \\
\text { gationen } \\
\mathscr{N}\end{array}$ & $\begin{array}{c}\text { sonstige } \\
\text { Schuld- } \\
\text { ver- } \\
\text { schrei- } \\
\text { bungen } \\
\mathscr{A}\end{array}$ & Aktien & $\begin{array}{c}\text { staat- } \\
\text { liche und } \\
\text { kommu- } \\
\text { nale } \\
\text { Anlethen } \\
\mathscr{N}\end{array}$ & $\begin{array}{c}\text { sonstige } \\
\text { Schuld- } \\
\text { ver- } \\
\text { schrei- } \\
\text { bungen } \\
\mathscr{M}\end{array}$ & Aktien \\
\hline Lebens- (Aktiengesellschaften. . & 382903 & 777 & ro & 28 & $\mathbf{I}$ & 10 & 172 & 1 & $\mathbf{r}$ \\
\hline versiche- $\{$ Gegenseitigheitsvereme. & 239300 & 832 & 38 & 30 & - & 5 & 95 & $(0,07)$ & - \\
\hline rung Belufsveremgungen. . & 46106 & 930 & 49 & 20 & - & - & I & - & - \\
\hline $\begin{array}{l}\text { Unfall- und Haftpflichtversicherungs- } \\
\text { gesellschaften }\end{array}$ & 93149 & 648 & 37 & . 20 & $(0,02 I)$ & 87 & 149 & 55 & 4 \\
\hline Viehver- ( Ahtiengesellschaften & 551 & 748 & - & 151 & -- & - & 101 & $\ldots$ & - \\
\hline sıcherung $\{$ Gegenseitıgheitsı ereine & 5811 & 675 & 148 & 170 & 5 & - & 2 & - & - \\
\hline Hagelver-( Abtiengesellschaften & 22328 & 849 & 4 & 28 & 2 & 115 & & - & - \\
\hline sıcherung Gegenseitigkeitsvereme & 12554 & 788 & 45 & 167 & - & 一 & - & - & - \\
\hline Feuerver-f Ahtiengesellschaften & 228192 & 724 & 29 & 47 & 3 & 11 & 107 & 72 & 7 \\
\hline sicherung Gegenseitigheitsvereme & 62055 & 743 & 139 & 114 & $\mathbf{I}$ & $(0,04)$ & 3 & - & - \\
\hline Glasver- (Ahtiengesellschaften & 2926 & 656 & I I & 36 & - & 48 & 247 & 2 & - \\
\hline su hes ung $\{$ Gegenseitiglseitsvereine & 295 & 632 & 20 & 221 & 121 & - & 6 & - & - \\
\hline Ruchversicherung Ahtiengesellschaften & 252981 & $39 \mathrm{I}$ & 25 & 41 & II & 107 & 191 & 124 & I Io \\
\hline Insgesamt & 1349151 & 701 & $31 i$ & 39 & 3 & 34 & 130 & 40 & 22 \\
\hline
\end{tabular}

Die

Vertellung des Gew inns

In der obigen Bilanz-Tafel sind beı jedem Versicherungssweige die Verluste, die einige Untemelıpungen zu verıeichnen liatten, gegen die Gewinne der anderen Gesellschaften verı echnet. Es ergibt sich so für die Gesamtheit der deutschen Versicherungsunternehmungen ein Rein-Gewinn von 292,191 Millionen Mark. Handelt es sich aber um die Verteilung des Gewinns, so kommt der durch die Bilanz ausgewiesene Gewinn aller derjenigen Gesellschaften in Betracht, die mit finanziell günstigem Erfolge gearbeitet haben, das sind 293,3ı Millionen Mark. Wie dieser Betrag verteilt worden ist. zeigt folgende Ubersicht:

\begin{tabular}{|c|c|c|c|c|c|c|c|c|}
\hline $\begin{array}{c}\text { Von dem Gewinne sind in } 1000 \mathscr{A l} \\
\text { abgefuhrt worden }\end{array}$ & $\begin{array}{c}\text { an dıe } \\
\text { gesetz- } \\
\text { lıche } \\
\text { Rucklage }\end{array}$ & $\begin{array}{c}\text { an } \\
\text { sonstige } \\
\text { Ruck- } \\
\text { lagen }\end{array}$ & $\begin{array}{c}\text { an den } \\
\text { Beamten- } \\
\text { wohl- } \\
\text { fahrts- } \\
\text { stock }\end{array}$ & $\begin{array}{c}\text { an die } \\
\text { Aktionare } \\
\text { oder } \\
\text { Garanten }\end{array}$ & $\begin{array}{c}\text { an Tan- } \\
\text { treme- } \\
\text { be- } \\
\text { rechtigte }\end{array}$ & $\begin{array}{c}\text { an die } \\
\text { Ver- } \\
\text { sicherten }\end{array}$ & $\begin{array}{l}\text { ander- } \\
\text { weit }\end{array}$ & $\begin{array}{l}\text { Vortrag } \\
\text { auf neue } \\
\text { Rechnung }\end{array}$ \\
\hline Lebenst ersicherung & 888 & 15800 & 786 & 8043 & 2930 & 154904 & I 455 & I 357 \\
\hline Unfall- und Haftpflichtversicherung & 7 & 641 & 496 & 6217 & 1706 & 5706 & I 192 & 2433 \\
\hline Viehversicherung & 185 & 179 & 33 & 25 & 65 & 64 & 28 & 47 \\
\hline Hagelversicherung & 727 & 566 & 247 & 2014 & 447 & 49 & 1022 & 227 \\
\hline Feuerversicherung usw.$\ldots$ & I 940 & 7990 & I 667 & 16037 & 2757 & 24227 & I 955 & 4995 \\
\hline Glasversıcherung & 18 & 280 & - & $35^{2}$ & 141 & 32 & 48 & 68 \\
\hline Ruckversicherung . . . . & 616 & I 529 & 272 & 9322 & I 054 & - & 675 & 6855 \\
\hline Zusammen & 4381 & 26985 & 3501 & 42010 & 9 100 & 184982 & 6375 & 15982 \\
\hline
\end{tabular}


Außerdem sind den Versicherten der Lebensversicherungsabteilungen verschiedener Aktiengesellschaften zusammen noch 5,846 Millionen vor dem Abschluß der Jahresrechnung als Gewinnanteil überwiesen worden, die in vorstehender Ubersicht nicht verrechnet sind.

Schließlich mag hier noch eine Gegenüberstellung der Beiträge und der Schäden der unmittelbar arbeitenden Unternehmungen, beide einschließlich der Anteile der Rückversicherer und des mittelbaren Geschäfts, bei den verschiedenen Versicherungszweigen Platz finden, die einerseits das ausländische Geschäft der deutschen Gesellschaften und anderseits das deutsche Geschäft der ausländischen Gesellschaften betreffen. Es sei aber besonders hervorgehoben, $\mathrm{da} ß$ aus den Endzahlen keineswegs Schlüsse auf die Verhältnisse des Versicherungsgeschäfts überhaupt gezogen werden dürfen; dazu fehlen die Zahlen für die ganz vornehmlich internationalen Betriebe der 'Transportversicherung und Das auslandische Geschsft der deutschen und das deutsche Geschaft der auslandischen Gesellschaften der Rückversicherung. Auch ist zu beachten, daß die Zahlen für I9I6 auch bei den deutschen Gesellschaften nicht vollständig sind, weil die Gesellschaften über ihr Geschäft im feindlichen Auslande nicht immer hinreichend unterrichtet sind, und daß bei den ausländischen Gesellschaften die englischen und die französischen in diesem Jahre wiederum fehlen.

\begin{tabular}{|c|c|c|c|c|}
\hline \multirow{2}{*}{ Versicherutgazweig } & \multicolumn{2}{|c|}{$\begin{array}{l}\text { Auslandisches Geschaft der } \\
\text { deutschen Gesellschaften }\end{array}$} & \multicolumn{2}{|c|}{$\begin{array}{l}\text { Deutsches Geschaft der aus- } \\
\text { landischen Gesellschaften }\end{array}$} \\
\hline & $\begin{array}{l}\text { Bestrage } \\
1000 \mathscr{M}\end{array}$ & $\begin{array}{l}\text { Schaden } \\
1000 \mathscr{A}\end{array}$ & $\begin{array}{l}\text { Beitrage } \\
1000 \mathscr{N}\end{array}$ & $\begin{array}{l}\text { Schaden } \\
1000 \mathscr{A}\end{array}$ \\
\hline 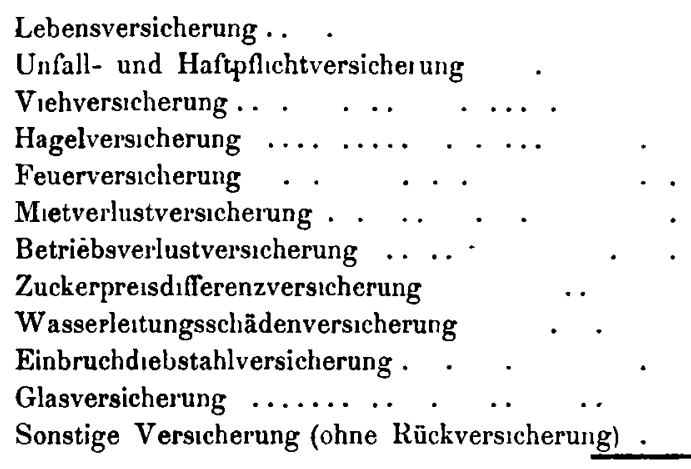 & $\begin{array}{r}70056 \\
7573 \\
211 \\
333 \\
63235 \\
3 \\
813 \\
-\quad 111 \\
1485 \\
782 \\
385 \\
\end{array}$ & $\begin{array}{r}3257 \\
3587 \\
165 \\
406 \\
39453 \\
-\quad 376 \\
-\quad 28 \\
453 \\
444 \\
107 \\
\end{array}$ & \begin{tabular}{rr|}
45 & 113 \\
13 & 280 \\
- & \\
- & \\
I 1276 \\
3 \\
$-\quad$ \\
8 \\
1 \\
1016 \\
24 \\
155 \\
\end{tabular} & $\begin{array}{r}36825 \\
-\quad 5859 \\
-\quad \\
-\quad 4578 \\
\\
-\quad 4 \\
-\quad 3 \\
513 \\
12 \\
295 \\
\end{array}$ \\
\hline 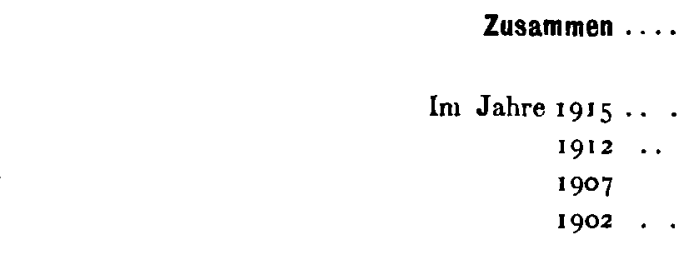 & $\begin{array}{r}144987 \\
141529 \\
160064 \\
109753 \\
90930\end{array}$ & $\begin{array}{ll}77 & 276 \\
80 & 288 \\
90 & 130 \\
60 & 002 \\
54 & 163\end{array}$ & \begin{tabular}{lll|}
70 & 889 \\
70 & 113 \\
98 & 375 \\
81 & 942 \\
6 & 1 & 38
\end{tabular} & $\begin{array}{l}48090 \\
51688 \\
62942 \\
55344 \\
33219\end{array}$ \\
\hline
\end{tabular}




\section{Gruppe I, Lebens- und Krankenversicherung}

Im Jahre 1916 standen auf dem Gebiete der Lebensversicherung und der Krankenversicherung 30 deutsche Aktiengesellschaften und eine große Zahl deutscher Gegenseitigkeitsvereine unter Reichsaufsicht.

Während 30 Aktiengesellschaften und 16 große Gegenseitigkeitsvereine ihren Geschäftsbetrieb für alle Kreise der Bevölkerung offen halten, versichert die Mehrzahl der Gegenseitigkeitsvereine nur die Angehörigen mehr oder weniger eng begrenzter Berufe. Von diesen Vereinen hat nur einer (der Preußische Beamtenverein in Hannover) einen so großen Versicherungsbestand gewonnen, daß man ihn den anderen Gegenseitigkeitsvereinen, die eine Beschränkung auf bestimmte Berufe nicht kennen, an die Seite stellen kann; deshalb ist er auch hier jenen allgemeinen Vereinen zugesellt worden. Von den anderen Gegenseitigkeitsvereinen für beschränkte Personengruppen sind in die Tafeln dieser Statistik nur einige der großeren Versicherungseinrichtungen von Berufsvereinigungen aufgenommen. Die große Mehrzahl von Pensions- und Sterbekassen, die vielfach nur die Versicherung der Angestellten bestimmter gewerblicher Firmen bezwecken, sind wie in den früheren Jahren aus den Tafeln ganz fortgelassen; sie werden weiter unten nur im Text und auch da nur ganz zusammenfassend behandelt.

Die größeren Pensions- und Sterbekassen gehören ihrem ganzen Betriebe nach eher zur kleinen als zur großen Versicherung; sie sind hier aber gesondert behandelt und als Versicherungseinrichtungen von Berufsvereinigungen zusammengefaßt. Die Bezeichnung "kleine Versicherung “ ist im folgenden lediglich für die kleine Versicherung der im allgemeinen allen Bevölkerungsklassen zugänglichen großen Unternehmungen verwendet.

Von allen dem Aufsichtsamt unterstehenden deutschen Lebensversicherungsvereinen auf Gegenseitigkeit sind in den Tafeln dieser Statistik 18 größere Gegenseitigkeitsvereine allgemeinen Charakters und 14 Versicherungseinrichtungen von Berufsvereinigungen behandelt.

Die Volksversicherungs-Aktiengesellschaft des Deutschnationalen HandlungsgehilfenVerbandes in Hamburg hat ihre Firma geändert in Deutschnationale Aktiengesellschaft für kleine Lebensversicherung in Hamburg.

Die Patria, Kranken- und Sterbegeld-Versicherungs-Bank, Aktıengesellschaft in Berlin, welche sich seit April I9I5 in Liquidation befindet, hat ihren Bestand an Sterbegeldversicherung mit Wirkung vom I. Januar 19 I 6 auf die Hamburg-Mannheimer Versicherungs-Aktien-Gesellschaft in Hamburg übertragen. Der Patria verbleibt demnach nur noch das Geschäft der Krankenversicherung. 
Von ausländischen Lebensversicherungsgesellschaften sind im Jabre 19 1 6 wiederum nur 17 in der Statistik behandelt, da 2 englısche und 2 französische Gesellschaften infolge des Krieges keine Geschäftsberichte eingereicht haben. Da ein Geschüftsbericht der Assicurazioni Generali in Triest auch für I9 6 nicht zu erlangen war, sind in den Tafeln dieser Statistik bei dieser Gesellschaft, wie schon im Vorjahre, durchweg die Zahlen für I 9 I 4 eingestellt worden.

Von den 30 deutschen Aktiengesellschaften betrieben im Jahre 1916 neben der Lebensversicherung 15 auch die Unfallversicherung, 15 die Haftpflichtversicherung, 5 die Feuerversicherung, 3 die Kautionsversicherung (in ganz bescheidenem Umfang), 4 die Transportversicherung und 4 die Versicherung gegen Vinbruchdiebstahl. Von den Gegenseitigkeitsvereinen hat nur einer (der Allgemeine Deutsche Versicherungs-Verein in Stuttgart) außer in der Lebensversicherung noch in der Unfall- und IIaftphlichtversicherung und in einigen kleinen Nebenzweigen Geschäfte gemacht.

Von den allen Kreisen der Bevölkerung zugänglichen deutschen Unternehmungen unter Reichsaufsicht sind im Jahre i916 tätig gewesen

\section{in der großen Versicherung}

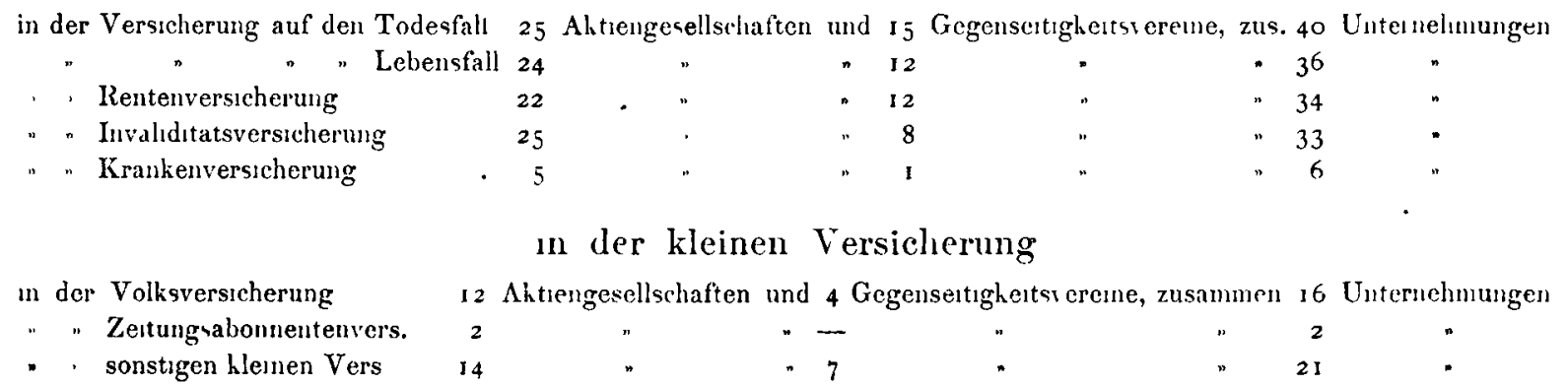

Von den 17 ausländischen Gesellschaften, über welche diese Statistik berichtet, haben im Deutschen Reiche gearbeitet sämtliche in der großen Versicherung auf den Todesfall, 16 in der Versicherung auf den Lebensfall, 16 in der Rentenversicherung, 6 in der Invaliditätsversicherung (Zusatzversicherung) und 4 in der Volksversicherung.

Vom Standpunkte der Statistik ist eine möglichst vollständige Trennung des großen Geschäfts von dem kleinen Geschäfte wünschenswert; denn der Betrieb ist verschieden, und die Versicherungen dienen zum Teil verschiedenen Zwecken

Nun haben nach den Rechnungsvorschriften diejenigen Versicherungsunternehmungen, deren Geschäftsbetrieb die sogenannte kleine Lebensversicherung (Volksversicherung, Arbeiter-, Sterbekassen- usw. Versicherung) ohne ärztliche Untersuchung oder mit unvollständiger ärztlicher Untersuchung in der Art umfaßt, daß für diesen Versicherungsbetrieb besondere geschäftliche Einrichtungen und besondere, von den sonstigen Geschäftsgrundsätzen der Unternchmung wesentlich abweichende allgemeine Versicherungsbedingungen, Tarife und Gebuhhrensätze eingeführt sind, für diesen Versicherungsbetrieb besondere Nachweisungen, namentlich auch über den Versicherungsbestand und seine Bewegung, sowie eine gesonderte Gewinn- und Verlustrechnung aufzustellen. Sofern aber die Unternehmungen für diesen Versicherungsbetrieb keine be- 
sonderen Einrichtungen haben, sind sie berechtigt, die kleine Versicherung mit der großen zusammen nachzuweisen.

Unter diesen Umständen ist es nicht moglich gewesen, die kleine Versicherung vollständig aus dem gesamten Geschäfte herauszuschälen. Bei einigen Unternehmungen konnte die Trennung nur beim Versicherungsbestande, nicht aber auch bei der Bewegung des Bestandes vollständig durchgeführt werden. Wo die Scheidung mit Hilfe der dem Aufsichtsamt eingereichten Übersichten oder der gedruckten Geschäftsberichte möglich war, ist sie vorgenommen worden, obwohl dadurch eine gewisse Ungleichmảßigkeit zwischen den einzelnen Abschnitten dieser Statistik entstehen mußte; es erschien besser, diese Ungleichmäßigkeit in den Kauf zu nehmen, als auf eine strenge Scheidung der beiden Betriebsarten vollständig zu verzichten Da, wo die Unternelımungen für die kleine Versicherung besondere geschäftliche Einrichtungen obengenannter Art geschaffen haben und wo mithin das kleine Geschäft von dem großen vollständig getrennt gehalten werden kann, wird in dieser Statistik die kleine Versicherung als "Volksversicherung", wo das nicht der Fall ist, als "sonstige kleine Versicherung " bezeichnet.

Der Beobachtungsstoff ist wie bisher folgendermaßen angeordnet.

Es ist zunächst der Versicherungsbestand am Ende des Berichtsjahres behandelt (Tafeln I 1 bis I 18); es folgt die Bewegung des Versicherungsbestandes (Tafeln I 19 bis I 28), dann die Gewinn- und Verlustrechnung (Tafeln I 29 bis I 36) und endlich die Bilanz für den Jahresschluß (Tafeln I 37 bis I 48).

In den einzelnen Abschnitten werden nacheinander berücksichtigt: die großen deutschen Unternehmungen (Aktiengesellschaften und Gegenseitigkeitsvereine), zuerst mit ihrer großen, dann mit ihrer kleinen Versicherung, danach die Versicherungseinrichtungen einiger größerer deutscher Berufsvereinigungen (Pensionskassen und Sterbekassen) und endlich die ausländischen Unternehmungen. Dabei wird von den deutschen Unternehmungen in den ersten drei Abschnitten immer das Lebensversicherungsgeschäft (inländisches und ausländisches) allein betrachtet, während die Bilanz für das Gesamtgeschäft (Lebensversicherung nebst allen Nebenzweigen) gegeben wird. Von den ausländischen Unternehmungen wird der Bestand und die Bewegung nur für das deutsche Geschäft gegeben; dagegen bezieht sich die Betriebsrechnung auf das ganze Lebensversicherungsgeschäft und die Bilanz auf das Gesamtgeschäft.

Hieran schließt sich eine Vergleichung des ausländischen Geschäfts der deutschen Unternehmungen mit dem deutschen Geschäfte der ausländischen Gesellschaften in den wichtigsten Punkten; endlich folgen einige Angaben über die dem Aufsichtsamt unterstehenden kleinen Pensions- und Sterbekassen.

\section{Der Versicherungsbestand am Ende des Jahres 1916}

Deutsche allgemeine Anstalten
Uber den gesamten Bestand der unter Reichsaufsicht stehenden deutschen Lebensversicherungsunternehmungen, die dem großen Publikum dienen, geben die Tafeln I 1 bis I 10 ausführlichen Aufschluß; hier sollen nur die hauptsächlichsten, für die Gesamtheit der Anstalten geltenden Ergebnisse mitgeteilt werden 
In der folgenden Zusammenstellung sind zunächst die Summen der von den einzelnen Unternehmungen Ende I 9 1 6 überlıaupt übernommenen Versicherungen wiedergegeben; diese Summen enthalten also alle Beträge doppelt, die von einer Gesellschaft. welche in unserer Statistik behandelt wird, versichert und bei einer ebensolchen Gesellschaft in Rückdeckung gegeben sind. Daneben ist angegeben, wieviel von je $1000 \mathscr{M}$ überhaupt übernommener Versicherung durchschnittlich für eigene Rechnung behalten worden ist.

\begin{tabular}{|c|c|c|c|c|c|c|}
\hline \multirow{2}{*}{ Versicherungsart } & \multicolumn{3}{|c|}{$\begin{array}{c}\text { Uberhaupt ubernommene Versicherungen } \\
\text { in } 10110 \mathscr{A}\end{array}$} & \multicolumn{3}{|c|}{$\begin{array}{c}\text { Von } 1000 \mathscr{A} \text { uberhaupt uber- } \\
\text { nommener Vers waren fur } \\
\text { eigene Rechnung behalten }\end{array}$} \\
\hline & $\begin{array}{l}\text { Aktien- } \\
\text { gesell- } \\
\text { schaften }\end{array}$ & $\begin{array}{l}\text { Gegen- } \\
\text { seltigheits- } \\
\text { vereme }\end{array}$ & z.usammen & $\begin{array}{l}\text { Abtien- } \\
\text { gesell- } \\
\text { schaften }\end{array}$ & $\begin{array}{c}\text { Gegen- } \\
\text { sertıgheits- } \\
\text { vereme }\end{array}$ & Jusammen \\
\hline Große Versicherung & & & & & & \\
\hline $\begin{array}{c}\text { Versicherung auf den Todesfall } \\
. " \text { Lebensfall }\end{array}$ & $\begin{array}{r}7548958 \\
465242\end{array}$ & $\begin{array}{r}5642549 \\
140375\end{array}$ & $\begin{array}{r}13191507 \\
605617\end{array}$ & $\begin{array}{l}917 \\
979\end{array}$ & $\begin{array}{r}987 \\
1000\end{array}$ & $\begin{array}{l}947 \\
984\end{array}$ \\
\hline $\begin{array}{l}\text { Rentenversicherung } \\
\qquad \text { Kle } 1 \text { ne Versicherung }\end{array}$ & 21823 & 5430 & 27253 & 939 & 999 & 951 \\
\hline Volhsrersicherung auf den Todesfall & I 437488 & 99142 & 1536630 & $98 \mathrm{I}$ & 1000 & 983 \\
\hline$" \quad " \quad$ Lebensfall & 567 & - & 567 & 667 & - & 667 \\
\hline Sonstıge kleıne Versicherung & $55 \circ 28$ & 151929 & 206957 & 994 & 972 & 978 \\
\hline
\end{tabular}

Die Rückversicherung spielt hiernach in der Lebensversicherung im ganzen nur eine bescheidene Rolle, zumal bei den Gegenseitigkeitsvereinen, bei denen sie fast nur in der großen Kapitalversicherung auf den 'Todesfall in Betracht kommt Bei der Lebensfall- und der Rentenversicherung, sowie bei der kleinen Versicherung tritt sie eigentlich nur da auf, wo Gesellschaften vertragsmäßig verpflichtet sind, von jeder von ihnen abgeschlossenen Versicherung einen bestimmten Hundertsatz in Rückdeckung zu geben (sogenannte Quotenrückversicherung).

Fragt man dann weiter, wie sich der Versicherungsbestand der deutschen Gesellschaften aus selbst abgeschlossenen und aus in Rückdeckung übernommenen Versicherungen zusammensetzt, so ersieht man aus den Tafeln I 3 bis I 7 und I 9 und 10 , daß die Gegenseitigkeitsvereine Ende 1916 nur wenige Versicherungen auf den Todesfall und vereinzelte Jahresrenten in Rückdeckung genommen und daß auch die Aktiengesellschaften im großen und ganzen nur wenig mittelbare Geschäfte abgeschlossen haben.

In der großen Todesfallversicherung haben die Unternehmungen noch nicht einmal halb so viel in Rückdeckung übernommen, wie sie in Rückdeckung gegeben haben; bei den anderen großen Versicherungen tritt die Rückversicherung ganz zurück.

In der Volksversicherung ist das mittelbare Geschäft kaum nennenswert.

Besondere Beachtung verdienen die Zahlen, die sich auf die unmittelbaren, selbst abgeschlossenen Versicherungen beziehen, weil darin ein Maßstab für den Versicherungsbedarf der Bevölkerung gefunden werden kann Wir geben davon sowohl die Zahl der Versicherungsscheine als auch die versicherten Summen und die durchschnittlich auf einen Schein entfallenden Beträge und setzen zum Vergleiche die Zahlen für I9 I daneben.

\footnotetext{
a) der ge-

samte Bestand und der fūr elgene Rechnung behaltene Tel]
} 
Gruppe 1

Unmittelbares Geschäft der deutschen allgemeinen Unternehmungen

Versicherungssummen in $1000 \mathscr{M}$, Jahresrenten und Durchschnittsbeträge in $\mathscr{N}$

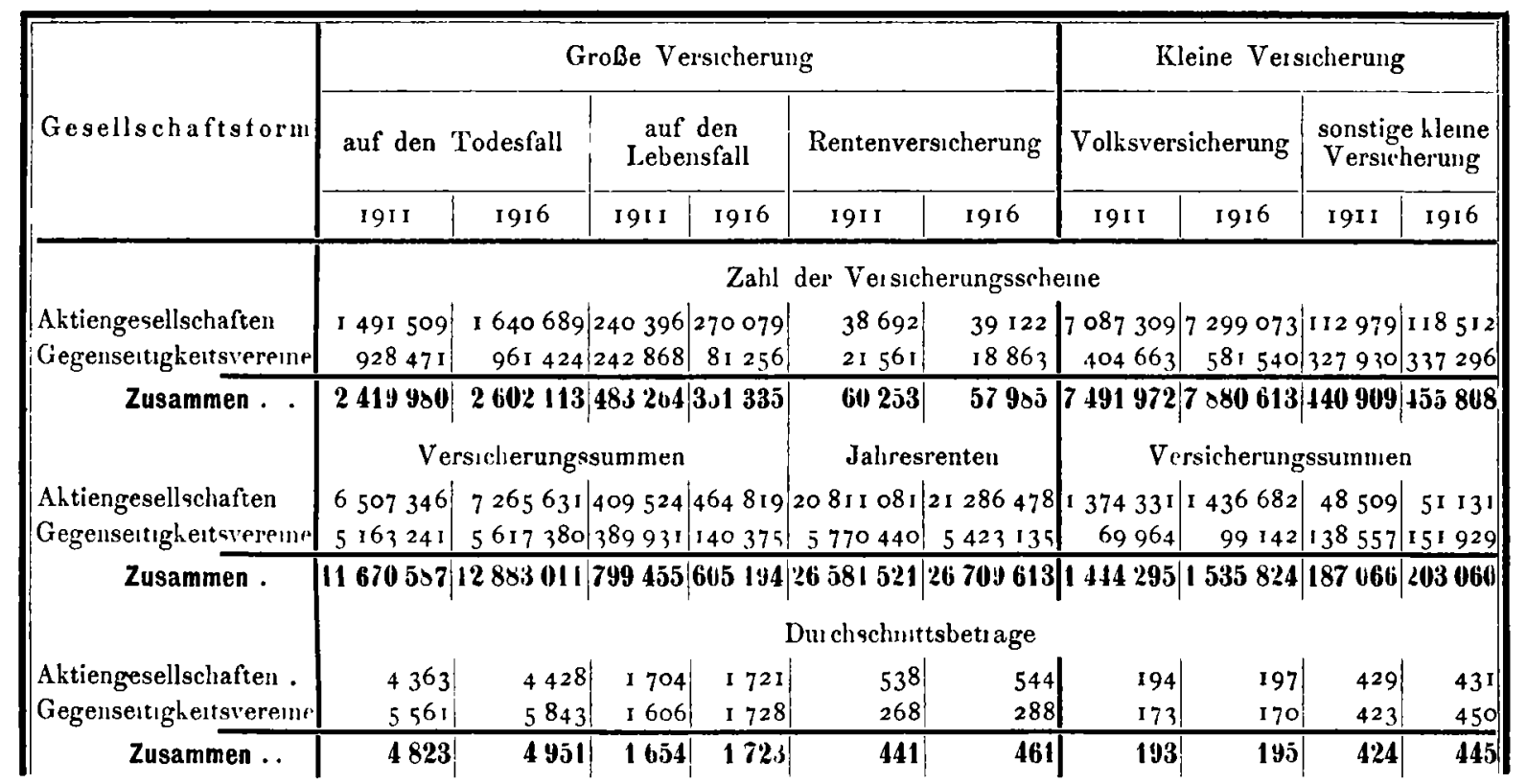

Die durchschnittlich auf einen Versicherungsschein entfallende Versicherungssumme ist in der großen Todesfallversicherung sowohl bei den Aktiengesellschaften wie auch bei den Gegenseitigkeitsvereinen gewachsen; sic ist bei den Gegenseitigkeitsvereinen fortdauernd höher als bei den Aktiengesellschaften. Der auf eine Versicherung auf den Lebensfall kommende durchschnittliche Betrag ist bei den Aktiengesellschaften etwas niedriger als bei den Gegenseitigkeitsvereinen; während der Durchschnittsbetrag bei den Aktiengesellschaften in den letzten Jahren etwas geschwankt hat, hat er bei den Gegenseitigkeitsvereinen ohne Unterbrechung zugenommen.

Beträchtlich größer waren die Durchschnittsbeträge bei der Gesamtheit der in den letzten Jahren ins Leben gerufenen offentlichen Versicherungsanstalten. Die 11 Anstalten (10 Provinzialanstalten und der Verband der öffentlichen Lebensversicherungsanstalten), die für ı 9 I 6 einen Jahresbericht veröffentlicht haben, hatten Ende 1916 im unmittelbaren Geschäfte zusammen 15554 'Todesfallversicherungen über $113706678 \mathscr{M}, 1197$ Versicherungen auf den Lebensfall über $2266361 \mathscr{N}, 247$ Versicherungen über $147176 \mathscr{N}$ Jahresrente und 87182 Volksversicherungen über $32739370 \mathscr{M}$ in Kraft. Die Durchschnittsbeträge stellen sich danach auf 7310 , auf 1893 , auf 596 und auf $376 \mathscr{N}$.

d) die Versicherungen mit und ohne Gicwinnbetelligung
In den 'Tafeln I 3 bis 7 und I 9 und 10 ist auch die Gewinnbeteiligung bei dem unmittelbaren Geschäfte behandelt. Daß Versicherungen mit Gewinnbeteiligung bei den Aktiengesellschaften verhältnismäßig weniger vorkommen als bei den Gegenseitigkeitsvereinen, liegt in der Natur der Gesellschaften. Genaueres hierüber ergibt für die letzten fünf Jahre die folgende Zusammenstellung.

Diese Zahlen zeigen durchweg einen sehr regelmäßigen Verlauf. In der Lebensfallversicherung und in der Rentenversicherung der Gegenseitigkeitsvereine sind die Versicherungen mit Gewinnbeteiligung während des betrachteten Zeitraums mit Ausnahme des letzten Jahres verhältnismäßig zurückgegangen, sowohl was die Zahl der Versicherungen als auch (wenigstens bei der Versicherung auf den Lebensfall) was die Höhe 


\begin{tabular}{|c|c|c|c|c|c|c|c|c|c|c|c|}
\hline \multicolumn{12}{|c|}{ Am Gewune waren im ummutelbaren Geschafte beteligt } \\
\hline \multirow{2}{*}{\multicolumn{2}{|c|}{ Versicherungsart }} & \multicolumn{5}{|c|}{ bei den Ahtiengesellschaften } & \multicolumn{5}{|c|}{ bei den Gegenseitigkettsi eremen } \\
\hline & & 1912 & 1913 & 1914 & 1915 & 1916 & 1912 & 1913 & 1914 & 1915 & 1916 \\
\hline Grobe Versicherung & & \multicolumn{10}{|c|}{ ron je 1000 Versicherungsschemen } \\
\hline Versicherung auf den Todesfall & - & 905 & 909 & 909 & 908 & 906 & 994 & 993 & 993 & 992 & 992 \\
\hline " $\quad "$ Lebensfall & . . & 330 & 324 & 317 & 545 & $54 \mathrm{I}$ & 900 & 897 & 892 & 775 & 779 \\
\hline $\begin{array}{l}\text { Rentenversicherung } . \\
\text { Kleıne Versichernng }\end{array}$ & & 3 & $?$ & $?$ & $?$ & $?$ & $73^{8}$ & 733 & 731 & 728 & 725 \\
\hline Volksversicherung .. . . & & 741 & 747 & 747 & 747 & 748 & 934 & 925 & $9: 7$ & 889 & 897 \\
\hline Sonstıge lleme Versicherung & & 309 & 333 & 332 & 320 & $33 \mathrm{I}$ & 933 & 935 & 936 & 935 & 936 \\
\hline Grobe Versicherung & & \multicolumn{10}{|c|}{ ron je 1001$) \mathscr{M}$ Versıcherungssumme (Jahresrente) } \\
\hline Versicherung auf den Todesfall & & 944 & 946 & 948 & 948 & 947 & 998 & 997 & 997 & 997 & 996 \\
\hline - $\quad$ "Lebensfall & $\cdot$ & 284 & 278 & 273 & 516 & $5: 7$ & 875 & 874 & 871 & 744 & 749 \\
\hline $\begin{array}{l}\text { Rentem ersicherung } \\
\text { Klene Versicherung }\end{array}$ & & 17 & $2 \mathbf{I}$ & 21 & 18 & $2 I$ & 621 & 623 & 629 & 629 & 628 \\
\hline Volhsversicherung . & . . & 819 & 829 & 829 & 823 & 828 & 972 & 969 & 966 & 952 & 956 \\
\hline Sonstıge hlene Versıcherung & . $\quad$. & 322 & 344 & 353 & 349 & 363 & 932 & 934 & 935 & 933 & 932 \\
\hline
\end{tabular}

der Versicherungssummen betrifft. Bei den Aktiengesellschaften ist infolge der erwähnten Fusionierung in der Lebensfallversicherung sowohl die Zahl der Versicherungen als auch die Höle der Versicherungssunmen, abgesehen von i 9 16, nicht unerheblich gestiegen; bei der Rentenversicherung hat die Gewinnbeteiligung zugenommen. Allerdings kommen solehe Versicherungen mit Anteil am Gewinne bei den Aktiengesellschaften überhaupt nicht allzuoft vor. In der großen Versicherung auf den Todesfall ist der Anteil der gewinnberechtigten Versicherungen bei den Gegenseitigkeitsvereinen ungeändert geblieben, bei den Aktiengesellschaften zeigt sich ein Rückgang. Bei der gesamten Volksversicherung ist eine Zunahme zu verzeichnen.

Die Versicherungen mit Gewinnbeteiligung sind, wenn man von der kleinen Versicherung absieht, am häufigsten bei der Versicherung auf den Todesfall; hier nälıern sich die Verlältniszahlen der Aktiengesellschaften denen der Gegenseitigkeitsvereine in bemerkenswerter Weise. Bei den Versiclverungen auf den Lebensfall findet sich die Gewinnbeteiligung schon seltener, wenn auch der Unterschied bei den Gegenseitigkeitsvereinen kleiner ist als bei den Aktiengesellschaften. Beachtenswert ist vielleicht, daß bei beiden Arten von Unternehmungen in der Todesfallversicherung die Scheine der Zall nach mit einem etwas geringeren Hundertsatz am Gewinn Anteil haben als die Versicherungssummen, während bei der Versicherung auf den Lebensfall das Entgegengesetzte der Fall ist. Die Versicherungen mit Anteil am Gewinne liegen also hinsichtlich der Höle der Versicherung beim Todesfall über, beim Lebensfall unter dem Durchschnitte. Rentenversicherungen werden von den Aktiengesellschaften nur ausnahmsweise mit Gewinnbeteiligung der Versicherten abgeschlossen; von den Gegenseitigkeitsvereinen geschieht das naturgemäß viel häufiger

Die Versicherung auf den Invaliditätsfall wird nur von wenigen Gesellschaften als selbständige Versicherung betrieben; zumeist tritt sie als Zusatzversicherung zu der gemischten Kapitalversicherung auf, indem fuir den Fall der Invalidität Beitragsfreiheit oder Beitragsfreiheit in Verbindung mit Gewährung einer Rente (meist 5 oder $10 \mathrm{v}$. H. des versicherten Kapitals) bis zum Ablaufe der Versicherung ausbedungen wird. Hin- 
sichtlich der hiermit verbundenen Unsicherheit in den Angaben über den Versicherungsbestand wird auf die Anmerkungen zu Tafel I 6 S. 20 Bezug genommen.

f) Kranken versicherung rungseınt chtungen von Berufs

vereinlgungen
Die Krankenversicherung eignet sich weniger als jede andere Versicherungsart zum Betriebe von einer Zentrale aus; sie erfordert, wenn sie ohne übergroße Gefährdung des Versicherers durchgeführt werden soll, eine weitgehende Beobachtung der Ansprüche erhebenden Versicherten, die in einfacher Weise nicht zu ermöglichen ist. 'Tatsächlich befaßten sich im Jahre 1916 denn auch nur wenige Gesellschaften mit der Krankenversicherung; von diesen Gesellschaften wickeln 2 nur das von andern Gesellschaften übernommene Geschäft $a b$, ohne selbst neue Versicherungen abzuschließen, vgl. Tafel I 7.

Von den für begrenzte Kreise bestimmten Versicherungsunternehmungen sind, wie schon oben erwähnt, in die Tafeln dieser Statistik die Versicherungseinrichtungen einiger größerer Berufsvereinigungen aufgenommen, obwohl diese Einrichtungen sehr erhebliche Unterschiede zeigen und sich deshalb nicht ohne Zwang gemeinsam behandeln lassen.

Von den fraglichen 14 Unternehmungen betrieben 10 die Versicherung auf den 'Todesfall (meist als Sterbekassen), davon drei auch die Versicherung auf den Lebensfall und drei auch die Rentenversicherung (meist Pensionsversicherung); die übrigen vier befaßten sich lediglich mit der Pensionsversicherung.

Im ganzen waren bei diesen Versicherungseinrichtungen Ende 19 I 6 in Kraft 273840 Versicherungsscheine über $200332297 \mathscr{M}$ auf den Todesfall (durchschnittlich $732 \mathscr{M}$ für eine Versicherung), 658 Versicherungsscheine über 2491793 \& $/$ auf den Lebensfall (durchschnittlich $3787 \mathscr{M}$ für eine Versicherung). In der Rentenversicherung liefen zu Ende des Berichtsjahrs 13038 Versicherungsscheine über $3644487 \mathscr{M}$ Jahresrente (durchschnittlich $280 \mathscr{M}$ Jahresrente für eine Versicherung). Außerdem bestanden noch 89343 Pensionsversicherungen mit fast ausschließlich steigender Rente.

Bei der einfachen Verwaltung der meisten derartigen Versicherungsvereine ist es erklärlich, daß sie bis auf die Deutsche Beamten-Lebensversicherung A.-G. in Berlin in der Rentenversicherung keine Versicherungen in Rückdeckung übernommen haben. Auch die in Rückdeckung gegebenen Beträge sind nicht eben beträchtlich (vgl. Anm. zu Taf. I 11 S. 26).

Von den ausländischen Versicherungsunternehmungen, die im Jahre 1916 im Deutschen Reiche Versicherungsgeschäfte betrieben haben, soll hier nur der Bestand des deutschen Geschäfts von I 7 Gesellschaften erörtert werden, wie er in den Tafeln I 12 bis I 18 erscheint. Dabei wird von einer Trennung zwischen Aktiengesellschaften und Gegenseitigkeitsvereinen abgesehen.

Wie vorhin bei den deutschen allgemeinen Unternehmungen, betrachten wir zunächst den gesamten übernommenen Bestand und den davon für eigene Rechnung behaltenen Teil.

\section{Es waren}

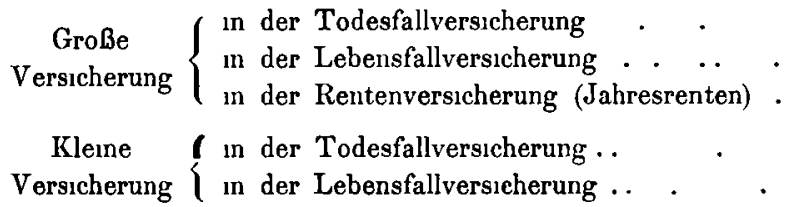

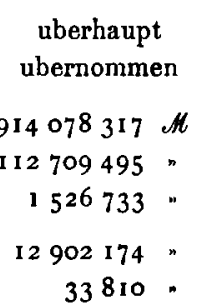


Danach haben die ausländischen Gesellschaften nur in der großen Todesfallversicherung und in der Rentenversicherung einen nennenswerten Betrag in Rückdeckung gegeben. Aus der kleinen Versicherung haben sie nur in der Todesfallversicherung einen geringen Betrag rückversichert (Quotenrückversicherung).

Utber das deutsche unmittelbare Geschäft der ausländischen Unternehmungen gibt sodann folgende Zusammenstellung Auskunft.

Deutsches unmittelbares Geschäft der ausländischen Unternehmungen

Versicherungssummen in $1000 \mathscr{M}$, Jahresrenten und Durchschnittsbeträge in $\mathscr{M}$

\begin{tabular}{|c|c|c|c|c|}
\hline & \multicolumn{3}{|c|}{ Große Versicherung } & \multirow{2}{*}{$\begin{array}{c}\text { Volks- } \\
\text { versicherung }\end{array}$} \\
\hline & $\begin{array}{c}\text { auf den } \\
\text { Todesfall }\end{array}$ & $\begin{array}{c}\text { auf den } \\
\text { Lebensfall }\end{array}$ & $\begin{array}{c}\text { Renten- } \\
\text { versicherung }\end{array}$ & \\
\hline & 1916 & 1916 & 1916 & 1916 \\
\hline Zahl der Versicherungsscheme & 148334 & $8 \mathrm{I} 487$ & 2356 & 54187 \\
\hline Versicherungssummen (Jahresrenten) & 890275 & 112642 & I 509021 & 12936 \\
\hline Durchschnttsbetrage & 6002 & I 382 & 700 & 239 \\
\hline
\end{tabular}

Die Durchschnittsbeträge sind bei den ausländischen Gesellschaften in der großen Todesfallversicherung merklich höher als bei den deutschen Anstalten. Das rührt jedoch daher, daß einige wenige Gesellschaften besonders hohe Beträge übernommen haben; es handelt sich nicht um eine Figentümlichkeit aller ausländischen Unternehmungen.

Ưber das Verhältnis der mit Gewinnbeteiligung der Versicherten abgeschlossenen Verträge zu den überhaupt abgeschlossenen Verträgen und über die Entwickelung dieses Verhältnisses in den letzten fünf Jahren ergeben sich bei Beschränkung auf das unmittelbare Geschäft folgende Zahlen. wobei indessen zu beachten ist, daß seıt r 9 I 4 zwei englische und zwei französische Gescllschaften fehlen.

Im deutschen unmittelbaren Geschäfte der ausländischen Unternehmungen waren am Gewinne beteiligt:

\begin{tabular}{|c|c|c|c|c|c|c|}
\hline \multicolumn{2}{|c|}{ Versicherungsart } & 1912 & 1913 & 1914 & 1915 & 1916 \\
\hline & & \multicolumn{5}{|c|}{ von Je I ooo Versicherungsscheinen } \\
\hline Große Todesfallversicherung & . & 862 & 868 & 870 & 869 & $87 \mathrm{I}$ \\
\hline " Lebensfallv ersicherung & . & 835 & 842 & 846 & 989 & 863 \\
\hline Rentenversicherung & . $\cdots$ & 43 & 41 & 39 & 37 & 36 \\
\hline Volksversicherung & ... & III & 117 & 116 & 108 & 90 \\
\hline & & \multicolumn{5}{|c|}{ von je i $000 \mathscr{A l}$ Versicherungssumme } \\
\hline Große Todesfallversicherung & & 911 & 914 & 916 & 915 & 914 \\
\hline " Lebensfallversicherung & . & 782 & 789 & 762 & 764 & 769 \\
\hline Rentenversicherung . .. & & 43 & 47 & 48 & 45 & 45 \\
\hline Volksversicherung ... & . & $3^{68}$ & 362 & 354 & 339 & 299 \\
\hline
\end{tabular}

Wie bei den deutschen Gesellschaften, ist auch bei den ausländischen Unternel1mungen die Gewinnbeteiligung derVersicherten bei der Todesfallversicherung am häufigsten. Bezüglich der Versicherungssumme ist die Gewinnbeteiligung der Versicherten bei den ausländischen Anstalten geringer als bei den deutschen Aktiengesellschaften. 
In der Volksversicherung schließen die ausländischen Gesellschaften bei uns verhältnismäßig sehr viel weniger Verträge mit Gewinnbeteiligung ab als die deutschen Unternelımungen.

\section{Die Bewegung im Versicherungsbestand im Jahre 1916}

Deutsche allgemeine Unternehmungen

Die Bewegung im Versicherungsbestande der deutschen Unternehmungen, die allen Kreisen der Bevölkerung offenstehen, ist in den 'Tafeln I 19 bis I 23 ausführlich behandelt. Dabei hat aber, wie schon oben bemerkt ist, die Trennung zwischen großer und kleiner Versicherung nicht so vollständig durchgeführt werden können wie bei der Beschreibung des Bestandes am Ende des Berichtsjahrs; die Bewegung der Sterbegeldversicherung war bei einigen Unternehmungen nicht aus der Bewegung der großen Versicherung auszuscheiden.

Die folgende Zusammenstellung gibt einen Uberblick uber die Bewegung der wichtigsten Versicherungsarten in dem gesamten Geschäfte der großen deutschen Unternehmungen; dabei ist bei der Volksversicherung und bei der sonstigen kleinen Versicherung die Todesfallversicherung mit der Lebensfallversicherung zusammengefaßt. In Betracht gezogen sind nur die selbst abgeschlossenen Versicherungen.

Der früher gewohnte Fortschritt ist weiter durch den Krieg unterbrochen worden; fast überall hat der Abgang den Zugang übertroffen; bei der Volksversiclıcrung der Gegenseitigkeitsvereine ist ein wesentlicher Reinzugang zu verzeichnen. Der Alogang ist

Bewegung im Versicherungsbestande der deutschen Unternehmungen

\begin{tabular}{|c|c|c|c|c|c|c|c|c|c|}
\hline & \multicolumn{2}{|c|}{$\begin{array}{c}\text { Versicherung } \\
\text { auf } \\
\text { den Todesfall }\end{array}$} & $\begin{array}{c}\text { Versicherung } \\
\text { auf } \\
\text { den Lebensfall } \\
\begin{array}{c}\text { Versiche- } \\
\text { rungs- } \\
\text { scheine }\end{array} \\
\end{array}$ & \multicolumn{2}{|c|}{\begin{tabular}{|c} 
Renten- \\
versicherung \\
$\begin{array}{c}\text { Versiche- } \\
\text { rung } \\
\text { scheme }\end{array}$ \\
\end{tabular}} & \multicolumn{2}{|c|}{$\begin{array}{l}\text { Volks- } \\
\text { versicherung }\end{array}$} & \multicolumn{2}{|c|}{$\begin{array}{l}\text { Sonstıge kleme } \\
\text { Versicherung }\end{array}$} \\
\hline \multicolumn{10}{|c|}{ Aktiengesellschaften } \\
\hline & \multicolumn{2}{|c|}{$\begin{array}{l}25 \text { Gesell- } \\
\text { schaften }\end{array}$} & $\begin{array}{l}24 \text { Gesell- } \\
\text { schaften }\end{array}$ & \multicolumn{2}{|c|}{$\begin{array}{l}22 \text { Gesell- } \\
\text { schaften }\end{array}$} & \multicolumn{2}{|c|}{$\begin{array}{l}12 \text { Gesell- } \\
\text { schaften }\end{array}$} & \multicolumn{2}{|c|}{$\begin{array}{l}\text { I1 Gesell- } \\
\text { schaften }\end{array}$} \\
\hline Bestand Ende 1915 & 1709754 & 7373336 & $290572: 493316$ & 39629 & 2 I 448 & 7364897 & I $44377 \mathrm{I}$ & 89434 & $343 \circ 3$ \\
\hline Zugang .... & 46156 & 260169 & $\begin{array}{llll}6414 & 15250\end{array}$ & I 739 & I 073 & 506787 & 122872 & 2892 & 1461 \\
\hline Abgang. & 80325 & 349904 & $26907, \quad 43747$ & 2246 & 1234 & 574539 & 130527 & 8710 & 2603 \\
\hline Remer Zugang & -34169 & $-\quad 89735$ & $-20493-28497$ & -507 & -161 & $-6775^{2}$ & -7655 & -5818 & $|-1142|$ \\
\hline Bestand Ende 1916 & $\left|16755^{85}\right|$ & 7283601 & $\left|\begin{array}{llll|}270 & 079 & 464 & 819\end{array}\right|$ & 39 I22 & 21287 & 7297145 & I 436 I16 & $83616 !$ & $|33161|$ \\
\hline \multicolumn{10}{|c|}{ Gegenseitigkeitsvereine } \\
\hline & \multicolumn{2}{|c|}{15 Vereme } & 12 Vereme & \multicolumn{2}{|c|}{12 Vereme } & \multicolumn{2}{|c|}{ \pm Vereme } & \multicolumn{2}{|c|}{7 Vereme } \\
\hline Bestand Ende 1915 & 976109 & 5629825 & $84674|146686|$ & 19479 & 5538 & 544 107 & 91352 & $33^{8783}$ & $|151572|$ \\
\hline Zugang. & 23957 & I93589 & 2983 & 515 & 184 & 79665 & 14601 & 5646 & 3981 \\
\hline Abgang. & 35918 & 204448 & $6401 \quad 12777$ & 1 I $3 I$ & 299 & $\begin{array}{lll}42 & 232\end{array}$ & $68 \mathrm{II}$ & 9857 & 5210 \\
\hline Remer Zugang ... & - II 961 & -10859 & $-3418-6311$ & -616 & -115 & 37433 & 7790 & $-42 \mathrm{II}$ & -1229 \\
\hline Bestand Ende 1916 & $964 \quad 148$ & 5618966 & \begin{tabular}{lll|ll|}
81 & 256 & 140 & 375
\end{tabular} & I 8863 & 5423 & 581540 & 99142 & $334572 \mid$ & $|\mathrm{I} 50343|$ \\
\hline \multicolumn{10}{|c|}{ Samtliche Unternehmungen } \\
\hline & $\begin{array}{r}40 \mathrm{U} \\
\text { nehm }\end{array}$ & $\begin{array}{l}\text { Jnter- } \\
\text { ungen }\end{array}$ & $\begin{array}{l}36 \text { Unter- } \\
\text { nehmungen }\end{array}$ & \multicolumn{2}{|c|}{$\begin{array}{l}34 \text { Unter- } \\
\text { nehmungen }\end{array}$} & \multicolumn{2}{|c|}{$\begin{array}{l}16 \text { Unter- } \\
\text { nehmungen }\end{array}$} & \multicolumn{2}{|c|}{$\begin{array}{l}18 \text { Unter- } \\
\text { nehmungen }\end{array}$} \\
\hline Bestand Ende 19I5 & 2685863 & 13003161 & $375246 \quad 640002$ & 59108 & 26986 & 7909004 & I 535123 & 428217 & $\left|\begin{array}{lll}1 & 85 & 875\end{array}\right|$ \\
\hline Zugang & $70 \begin{array}{llll}0 & 1 & 3\end{array}$ & $45375^{8}$ & 21716 & 2254 & 1257 & $5^{86} 45^{2}$ & I 37473 & 8538 & 5442 \\
\hline Abgang & II 6243 & $55435^{2}$ & \begin{tabular}{ll|ll|}
33 & 308 & $5^{6}$ & 524 \\
\end{tabular} & 3377 & I 533 & 616771 & I $3733^{8}$ & 18567 & 7813 \\
\hline er Zugang & -46 I 30 & - 100594 & $-23911)-34808$ & $-1 \quad 123$ & -276 & -30319 & 135 & -10099 & -2371 \\
\hline Bestand Ende 1916 & 2639733 & 12902567 & $\begin{array}{lllll}351 & 335 ! & 605 & 194\end{array}$ & 57985 & 26710 & 7878685 & I 535258 & $\mid 418188$ & $|183504|$ \\
\hline
\end{tabular}


gegen die Vorjahre I9I4 und I9I5 kleiner, der Zugang im allgemeinen größer gewesen. Der Krieg hat durch weitere Kinberufung zum Heeresdienste große Lücken in das Netz der Vertreter und Vermittler der Gesellschaften gerissen und die Kreise, in denen vornehmlich für Lebensversicherungen geworben wurde, gelichtet. Die Ausscheidenden kamen zunächst für die Versicherung nicht mehr in Betracht.

Wegen der großen Bedeutung, die den verschiedenen Ursachen des Abgangs von Versicherungen zụkommt, sind in unsern Tafeln für die Hauptversicherungsarten nicht nur die absoluten Zahlen für die Häufigkeit der einzelnen Ursachen angegeben, sondern es ist auch berechnet, wieviel bei den selbst abgeschlossenen Versicherungen von 1000 Scheinen oder von $1000 \mathscr{M}$ Versicherungssumme des durchschnittlichen Bestandes durch Tod, wieviel durch Ablauf usw. in Wegfall gekommen sind.

Mehr als in andern Zeiten verdienen während des Kriegs diese Zahlen unsere Aufmerksamkeit. Zu Friedenszeiten schienen sie von Jahr zu Jahr fast unveränderlich; jetzt bewegen sie sich, vornehmlich natürlich in der Versicherung auf den Todesfall. Wir geben daher folgende kurze Zusammenstellung, die für sich selber spricht.

\begin{tabular}{|c|c|c|c|c|c|c|c|}
\hline \multirow{2}{*}{\multicolumn{2}{|c|}{ GroßeVersicherung auf den Todesfall }} & $\begin{array}{l}\text { Abgang d } \\
\text { Ver- } \\
\text { scherungs- } \\
\text { scheine }\end{array}$ & $\begin{array}{l}\text { Ireh Tod } \\
\text { Summen }\end{array}$ & $\begin{array}{l}\text { Storno mit } \\
\text { Ver- } \\
\text { scherungs- } \\
\text { scheme }\end{array}$ & $\begin{array}{l}\text { Vergutung } \\
\text { Sumnen }\end{array}$ & $\begin{array}{l}\text { Storno ohne } \\
\text { Ver- } \\
\text { sicherungs- } \\
\text { scheine }\end{array}$ & $\begin{array}{l}\text { Vergutung } \\
\text { Sumrnen }\end{array}$ \\
\hline & & & & & & & \\
\hline \multirow[t]{3}{*}{ Aktiengesellschaften. . } & . ... 1914 & 15 & 14 & 15 & 18 & 23 & 23 \\
\hline & I9I 5 & 21 & 18 & 16 & 18 & 15 & 17 \\
\hline & 1916 & 17 & 15 & 12 & 14 & 7 & 7 \\
\hline \multirow[t]{3}{*}{ Gegenseitigheitsvereine } & .1914 & I 8 & 18 & 8 & 9 & 9 & 7 \\
\hline & 1915 & 22 & 20 & 6 & 8 & 5 & 5 \\
\hline & 1916 & 18 & I 7 & 5 & 5 & 3 & 3 \\
\hline \multirow[t]{3}{*}{ Aktiengesellschaften .. } & .1914 & I I & 10 & 7 & 27 & 71 & 89 \\
\hline & 1915 & 14 & 14 & 5 & 35 & 44 & 50 \\
\hline & 1916 & I3 & I 3 & 4 & 18 & 23 & 29 \\
\hline \multirow[t]{3}{*}{ Gegenseitigkeitsvereme . } & $\ldots .1914$ & Io & Io & 35 & 35 & 120 & 142 \\
\hline & 1915 & 14 & I4 & 23 & 25 & 61 & 66 \\
\hline & 1916 & 12 & I 3 & 13 & 14 & 26 & 29 \\
\hline
\end{tabular}

Wenn man bei den Versicherungseinrichtungen der größeren Berufsvereinigungen (Pensionskassen und Sterbekassen) diejenigen Kassen außer Betracht lảßt, bei denen die Jahresrenten als veränderlich nicht angegeben werden können, erhält man folgendes Bild von der Bestandsänderung im Berichtsjahre:

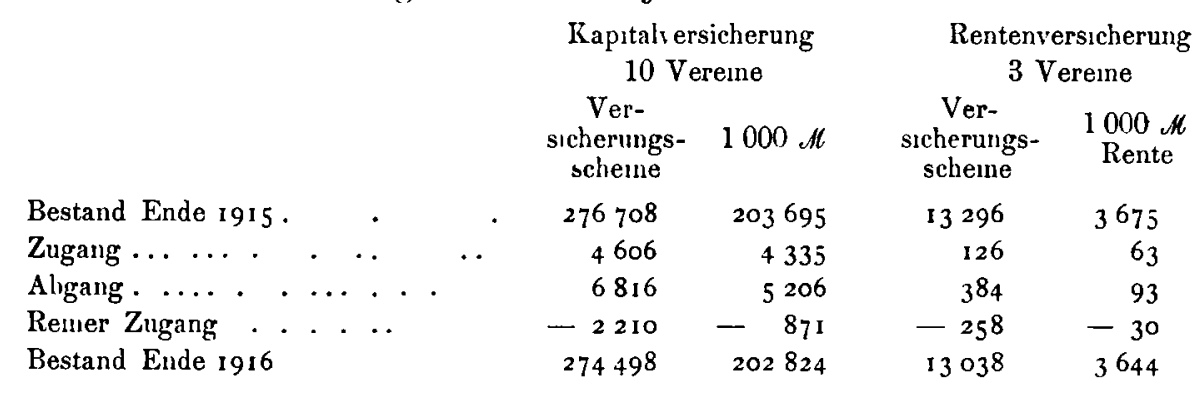

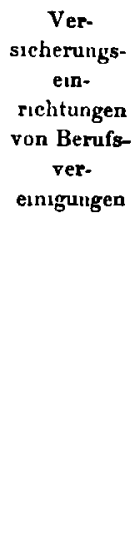

Die Bewegung im deutschen Versicherungsbestande der ausländischen Lebensversicherungsunternehmungen ist in den Tafeln I 25 bis I 28 ausfülırlich dargestellt. Aus diesen Tafeln ist folgende Utbersicht hergeleitet: 
Bewegung im deutschen Versicherungsbestand der ausländischen Gesellschaften

\begin{tabular}{|c|c|c|c|c|c|c|c|c|}
\hline & \multicolumn{2}{|c|}{$\begin{array}{l}\text { Versicherung auf } \\
\text { den Todesfall } \\
17 \text { Gesellschaften }\end{array}$} & \multicolumn{2}{|c|}{$\begin{array}{l}\text { Versicherung auf } \\
\text { den Lebensfall } \\
16 \text { Gesellschaften }\end{array}$} & \multicolumn{2}{|c|}{$\begin{array}{c}\text { Renten- } \\
\text { versicherung } \\
\text { 16 Gesellschaften }\end{array}$} & \multicolumn{2}{|c|}{$\begin{array}{c}\text { Volks- } \\
\text { versicherung } \\
3 \text { Gesellschaften }\end{array}$} \\
\hline Bestand Ende 1915 . . & 148749 & 909379 & 80853 & I I 4355 & 2156 & I 464 & 47674 & 11817 \\
\hline Zugang.. . .. & 7 I 25 & 22687 & 5684 & 6919 & 93 & 100 & I 1256 & 2033 \\
\hline Abgang .. & $735^{\circ}$ & 41762 & $5 \circ 50$ & 8632 & 93 & 55 & 4933 & 943 \\
\hline Remer Zugang . .. & 225 & -19075 & 634 & -1713 & - & 45 & 6323 & 1090 \\
\hline Bestand Ende 1916 & 148524 & 890304 & 81487 & I 2642 & 2156 & I 509 & 53997 & I 2907 \\
\hline
\end{tabular}

Uber die Häufigkeit der verschiedenen Arten des Abganges seien hier die folgenden Zahlen aufgeführt, die den oben für die deutschen Unternehmungen angegebenen Zahlen entsprechen.

\begin{tabular}{|c|c|c|c|c|c|c|c|c|c|c|}
\hline \multirow[b]{3}{*}{ Große } & \multirow[b]{3}{*}{ Versicherun } & \multicolumn{5}{|c|}{$\begin{array}{c}\text { Abgang in Tausendsteln des durch } \\
\text { Abgang durch Tod }\end{array}$} & \multicolumn{2}{|c|}{$\begin{array}{l}\text { chnittlichen Bestal } \\
\text { Stomo mit Vergütung }\end{array}$} & \multirow{2}{*}{$\begin{array}{l}\text { Storno ohne } \\
\text { Versicherungs- } \\
\text { scheine }\end{array}$} & \multirow{2}{*}{$\begin{array}{l}\text { Vergütung } \\
\text { Sumnten }\end{array}$} \\
\hline & & & & \multicolumn{2}{|c|}{$\begin{array}{c}\begin{array}{c}\text { Versicheriungs- } \\
\text { scheine }\end{array} \\
\text { - }\end{array}$} & \multirow{2}{*}{$\begin{array}{c}\text { Summen } \\
16\end{array}$} & \multirow{2}{*}{$\begin{array}{l}\text { Versicherungs- } \\
\text { scheine } \\
17\end{array}$} & \multirow{2}{*}{$\begin{array}{c}\text { Summen } \\
23\end{array}$} & & \\
\hline & & Ig auf den & Todesfall & 1914 & 15 & & & & 27 & I 5 \\
\hline - & " & • & . & 1915. & 19 & 17 & I 5 & 17 & 21 & 12 \\
\hline$"$ & " & $"$ & $"$ & 1916 & 16 & 16 & 12 & I6 & 9 & 4 \\
\hline Volksv & ersıcherung & 1914 & & & 12 & 12 & 19 & 20 & 105 & 95 \\
\hline & . & 1915 & . & & I 4 & 14 & 15 & 18 & 96 & 82 \\
\hline & $*$ & 1916 & & & 14 & 13 & 16 & 16 & 60 & 39 \\
\hline
\end{tabular}

\section{Die Betriebsrechnung für 1916}

Allgemenes Die Gewinn- und Verlustrechnung, wie sie in den Tafeln I 29, 31, 33 und 35 gegeben ist, unterscheidet sich von den Aufstellungen, welche die Unternehmungen nach dem Formular L 1 der Rechnungsvorschriften dem Aufsichtsamt einzureichen haben, dadurch, daß die veränderlichen Rücklagen (Prämienreserve, Prämienüberträge, Rücklagen für schwebende Versicherungsfälle, Gewinnrücklagen und sonstige Rücklagen) nicht mit ihren Beständen zu Anfang des Jahres in Einnahme und mit ihren Werten am Ende des Jahres in Ausgabe gestellt sind, sondern daß nur die durch den Jahresbetrieb hervorgerufenen Änderungen nachgewiesen sind; denn nur diese Änderungen beeinflussen das Ergebnis der Jahresrechnung; außerdem wird die Rechnung durchsichtiger. Der Stand der Rücklagen am Ende des Jahres findet sich in der Bilanz. Sodann ist der Vortrag aus dem Überschusse des Vorjahrs hier fortgelassen worden, um das Ergebnis des Geschäftsbetriebs lediglich aus dem Berichtsjahre zu erhalten.

Die Betriebsrechnung bezieht sich auf das ganze (deutsche und ausländische) Lebensversicherungsgeschäft der Unternehmungen. Für die Volksversicherung ist bei den deutschen Gesellschaften eine besondere Betriebsrechnung (Tafel I 31) aufgestellt; die sonstige kleine Versicherung und bei den ausländischen Gesellschaften auch die Volksversicherung ist mit dem Geschäft in der großen Versicherung zusammen verrechnet (Tafel I 29 und 35) Auch sind beim Atlas, bei der Wilhelma und bei der Bayer. V. B. die geringen Einnahmen und Ausgaben der Kautionsversicherung in der allgemeinen Betriebsrechnung für das gesamte Lebensversicherungsgeschäft enthalten (vgl. die Anmerkungeh 1 und 2 zu Tafel I $30 \mathrm{~S} 92$ und 93) 
Die in den Ubcisicliten uber die Cewinn- und Verlustrcclınung nur im Ganzen eingestellten Beiträge und Vergütungen der Ruckversicherer einerscits und die Zahlungen für selbst algeschlossene und für in Rückdeckung übernommene Versicherungen sowie die Rückversicherungsbeiträge anderseits sind fur die deutsehen Unternohmungen in den Tafeln I 30,32 und 34 erläutert. Für die auslindischen Gesellschaften sind die entsprechenden Angaben nur für den deutschen Geschäftsbetrieb zusammengestellt (Tafel I $3(6)$.

In der Lebensversicherung ohne Volksversicherung haben bei den deutschen Unternelimungen betragen:

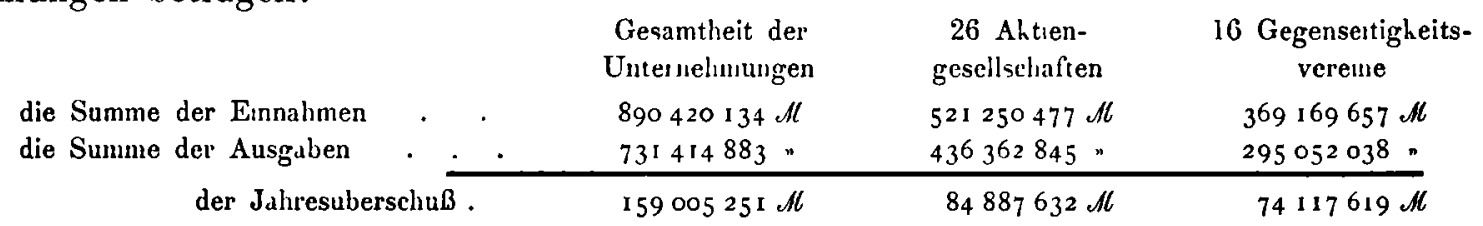

Deutsche aligemerne Unternchmungen

Das Geschäftsjahr I916 hat 25 Aktiengesellschaften in der großen Lebensversicherung einen Überschuß von zusammen $85002210 \mathscr{A}$ gebracht; eine Gesellschaft, die übrigens nur Krankenversicherung (auch mit Finschluß von Sterbegeld) betricben hat, hat einen Verlust von $114578 \mathscr{A l}$ ausgewiesen. Bei den 16 Gegenseitigkeitsvereinen ist ein Gewinn von zusammen $7+117619 \mathscr{H}$ erzielt worden. Von dem durch die Bilanz ausgewiesenen Gewinn und dessen Verteilung wird unten gesprochen werden.

Für die Volksversicherung ergeben sich folgende Zahlen:

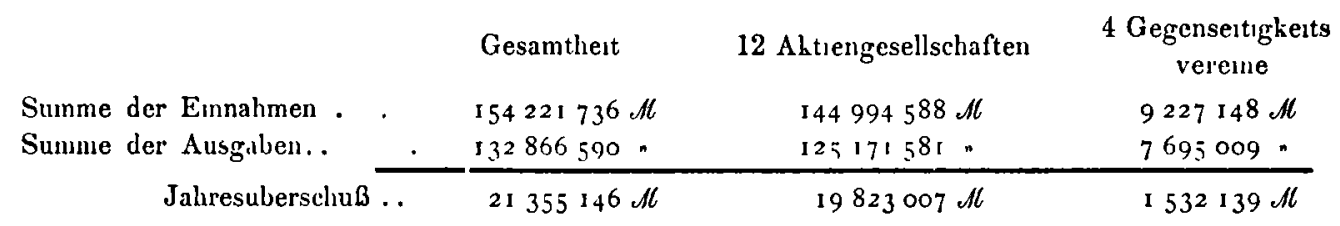

Von den 12 Aktiengesellschaften, deren Betricbsrechnung für das Volksversicherungsgeschäft in Tafel I 31 aufgestellt ist, haben 11 im Jahre I 916 mit einem Gewinne von $19828870 \mathscr{A l}$ gearbeitet, während eine einen Verlust von 58 (3:3) $\mathscr{A}$ ausweist. Die vier Gegenseitigkeitsvereine haben einen Uberschuß von zusammen $1532139 \mathscr{A l}$ erziclt.

Für eingetretene Versicherungsfalle des Geschifftujalırs aus selbst abgeschlossenen Versicherungen wurden im Berichtejahr in der großen Versicherung von der Gesamtheit der Unternelımungen 89,1 v. II. der fällig gewordenen Summen ausbezalılt und 10,9 v. II.zurïckgestellt; im besonderen ron den Aktiengesellschaften \$9,2 und 10,8 v. H., von den Gegenseitigkeitsvereinen 88,8 und 11,2 v. H In der Volksversicherung wurden von der Gesamtheit der Unternehmungen 93,74 v. II. aller fällig gewordenen Versicherungssummen gezahlt und 6,20 v. II. zuruckgestellt; im besonderen bei den Aktiengesellschatten 93,74 und 6,26 v. H, bei den Gegenseitigkeitsvercinen 93,80 und 6,20 v. H. Bei Vergleichen mit den früheren Jahren ist zu beachten, daß die Zahlungen aus Versicherungsfallen bei der Victoria seit 1909 die mit den Versicherungssummen ausgezahlten Gewinnanteile nicht melır enthaltcl1; diese erheblichen Beträge (im Berichtsjahre nach Anmerkung' ${ }^{4}$ ) S. 99: $1519800^{\circ} \mathscr{M}$ ) sind vielmelı bei den an die Versicherten ausgezahlten Gewinnanteilen verrechnet 
Versicherungseisuclitungen vin Berufarerenusuugen

Aus. Intrdische Gusellscharten

Uber die Gewinn- und Verlustrcchnung der in diese Statistik anfgenommenen Versicherungacinrichtungen der größeren Berulsvereinigungen geben die Tafeln I33 und I $3 \pm$ Aurschluß.

Daraus sind folgende Zahlen zu entnelimen:

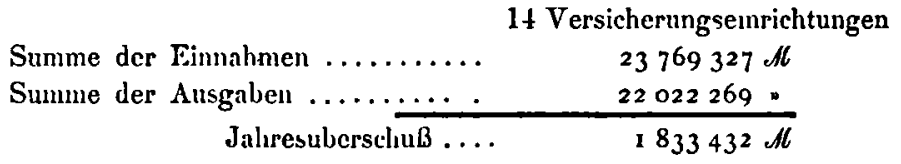

Einen Fchlbetrag haben im Berichtsjahre zwei dieser Anstalten mit $86374 \mathscr{M}$ ausgewiesen; die übrigen 12 Anstalten crzielten einen Ưberschuß von zusammen $1747058 \mathscr{H}$.

Zur Beurteilung des Geschäftsbetriebs derjenigen ausländischen Lebensversicherungsgescllschaften, über welche diese Statistik berichtet, ist in Tafel I35 die Gewinnund Verlustrechnung für das ganze inländische und ausländische Geschäff möglichıst nach densclben Grundsätzen dargestellt, die bei den deutschen Gesellschaften angewandt worden sind. Daneben sind aber in Tafel I 36 die Einnalımen und Ausgahen der ausländischen Gesellschaften allein aus dem deutschen Gंeschäfte zusammengestellt. lis haben betragen die Einnahmen an Beiträgen im deutschen Geschäft insgesamt $45112995 \mathscr{M}$. Daron kommen $44185934 \mathscr{M}$ oder 98,0 v. H. aut selbst alogeschlossene Versicherungen und 9.2706i $\mathscr{A l}$ oder 2,0 v. II. auf die in Rückdechung übernommenen Versiclıcrungen. Für Versicherungsleistungen einschließlich der Zahlungen für vorzeitig aufgelöste Versicherungen waren für das deutsche Geschäft bereitzustellen insgesamt $368.5098 \mathscr{M}$, davon $36181176 \mathscr{M}$ oder 98,3 v. II. für selbst abgeschlossene Versicherungen und $643922 \mathscr{A}$ oder 1,7 v. II. für in Rückdeckung übernommene Versicherungen.

\section{Die Bilanz für den Schluß des Jahres 1916}

Deutsche allge uciue Unternehmunge
Eine Bilanz ist in dieser Statistik grundsätzlich für das Gesamtgeschäft einer jeden Gesellschinft aufyestellt und bei derjenigen Gruppe nachgewiesen, zu der das Unternehmen seinem Ilaupthctriebe nach gehört.

Dementsprechend sind in Tafel I 37 die Bilanzen für 28 Alitiengesellschaften und 17 Gegenscitigkcitsvereine aufgefiihrt; die Gesamtbilanz schließt für Ende I 916 bei den Aktiengesellschaften mit $4382361094 \mathscr{H}$ und bei den Gegenseitigkeitsvereinen mit $2496864079 \mathscr{M}$ ab; als Ilauptposten sind folgende Beträge zu verzeichnen:

\begin{tabular}{|c|c|c|}
\hline \multicolumn{2}{|c|}{28 Ahtiengesellschaften } & \multirow{2}{*}{17 Gegensertigkertsvereine } \\
\hline Verpflichtungen der Ahtıonäre oder Garanten & $119956221 \mathscr{A L}$ & \\
\hline$\ldots \ldots \ldots \ldots \ldots \ldots \ldots$ & $22088944 I$ & 88826902 \\
\hline Sicherhetsdarlehen an Beamte................. & 168753 & 860625 . \\
\hline Kapitalaulagen $\ldots \ldots \ldots \ldots \ldots \ldots \ldots, \ldots \ldots \ldots \ldots$ & $399882 \mathrm{I}$ OOI & 2380199306 \\
\hline 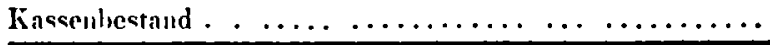 & 3141830 & 2779778 \\
\hline \multicolumn{3}{|l|}{ Verbindlichiceiten } \\
\hline Ahtien- oder Garantichapital................. & $162672857 \mathscr{H}$ & $427778 \mathscr{M}$ \\
\hline Gesetsliche Ruchlage $\ldots \ldots \ldots \ldots \ldots \ldots \ldots \ldots \ldots \ldots$ & 16456398 & 12264382 \\
\hline Sunder úchlagen $\ldots \ldots \ldots \ldots \ldots \ldots \ldots \ldots$ & 156574595 & 49461931 \\
\hline Gew mnrü klıge der Versicherten............ & $3829+4473$. & 212475484 \\
\hline Guthalien von Versichicrungsunternehmungen .......... & 18192905 & 761408 \\
\hline B.ırstrherheiten $\ldots \ldots \ldots \ldots \ldots \ldots \ldots \ldots \ldots \ldots \ldots$ & 1830792 & 862678 . \\
\hline Prännicnreserı en und Prämicnühırträge ..... & 3270928927 & 2029930923 " \\
\hline Rüchlage für schwebende Versıcherungsfälle . ....... & 59899017 & 28988057 \\
\hline
\end{tabular}


Die hier aufgeführten Kapitalanlagen verteilen sich auf folgende Wertarten (Tafel I 38):

\begin{tabular}{|c|c|c|c|c|c|c|c|}
\hline \multirow[b]{2}{*}{$\begin{array}{lllllll}\text { Grundbesitz } & \ldots & \ldots & \ldots & \ldots & \ldots & \ldots\end{array}$} & \multicolumn{3}{|c|}{29 Ahtiengescllschaften } & \multicolumn{4}{|c|}{17 Gegensestigheitsi erene } \\
\hline & 93623719 & $\mathscr{A l}$ oder & $2,4 \%$ & 18777624 & Al oder & 0,7 & $\%$ \\
\hline 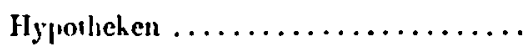 & 3031099298 & $n \quad \infty$ & 75,8 & I 736384561 & $\infty \quad \infty$ & 73.0 & , \\
\hline Dullehen an offentliche Körperschaften & 201707093 & • & $50 \%$ & 185986576 & 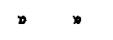 & 7,8 & $\rtimes$ \\
\hline Danlehen auf Versicherungen $\ldots . .$. & 315079553 & » & 7,9 ע & 211576009 & " & 8,9 & $\mathbf{p}$ \\
\hline Datlehen auf Wertpapiere... .... . & 447073 & • & $(0 \circ 1)$. & I 855472 & * & $\mathbf{o}, \mathbf{I}$ & " \\
\hline Werrpapiere $\ldots \ldots \ldots \ldots \ldots \ldots$ & 35686 I 67 I & » & $89 \%$ & 223324099 & $n$ & 9,4 & " \\
\hline Wechsel $\ldots \ldots \ldots \ldots \ldots \ldots \ldots \ldots$ & 2594 & $\because(0$, & $, 00004)$ & $229+965$ & $n$ & $0, \mathbf{r}$ & ע \\
\hline Sonstıge Anlagen . $\quad \ldots \ldots \cdot \ldots$ & 一 & & - & - & & - & \\
\hline
\end{tabular}

Vergleicht man diese Zahlen mit denen des Vorjahrs, so erlä̈lt man folgendes Bild: Bei den Aktiengesellschaften haben zugenommen der Wert des Grundbesitzes um $688332 \mathscr{M}$, der Ilypothekenbestand um $1623526 j \mathscr{M}$, die Darlehen an öftentliche Körperschaften um 93 $706053 \mathcal{M}$, die Darlehen auf Versicherungen um $5196014 \mathscr{M}$, die Wertpapipre um $48455170 \mathscr{H}$ (der Nennwert um rund 55 Millionen Mark); abgenommen haben dagegen die Darlehen auf Wertpapiere um $1860171 \mathscr{A l}$ und der Bestand an Wechseln um $5740 \mathscr{M}$. Bei den Gegenseitigkeitsvereinen haben zugenommen der Wert des Grundbesitzes um $962(1)(; \mathscr{H}$, die Darlelien auf Versicherungen um $3360381 \mathscr{A}$, die Wertpapiere um $67864484 \mathscr{N}$ (der Nennwert um rund 72 Millionen Nark), der Bestand an Wechseln um $1045533 \mathscr{M}$; abgenommen haben dagegen der Hypothekenlestand um $132786 \mathscr{M}$, die Darlehen an öftentliche Köperschaften um $2025024 \mathscr{A}$, die Darlehen auf Wertpapicre um $792717 \mathscr{M}$ Bedeutend mehr als im vorhergehenden Jahıre haben zugenommen die Darlehen an öffentliche Körperschatfen (um 85 Millionen). Weniger als im Vorjalire haben zugenommen die Darlehen auf Versicherungen und die Wertpapiere (sogar um 71 Millionen nach dem Nennwert). Ferner die Ilypotheken (um y Mullionen). Dem Kapitalmarkte haben die allgemeinen deutschen Lebensversicherungsunternehmungen zugefühırt: 1,6j Millionen Mark für Grundlbesitz, 16,10 Millionen fïr Ilypotheken, 92, is Millionen für Darlehıen an öffentliche Körperschaften, 116,32 Millionen für Wertpapiere und für Wechsel 1,04 Millionen, zusammen 2:7, i9 Millionen gegenüber 226,47 Millıonen im Vorjahıre.

Die Prëmienreserven und die Prämienüberträge sind in Tiffel I 39 nach Versicherungsarten getrennt angegeben. Hicrzu bedarf es indessen noch folgender Frlïuterungen. Nach $§ 58$ des Versicherungsaufsichtsgesetzes soll bei Rückversicherungen die Prümienreserve von dem rückversicherten Unternehmen berechnet, aufbewalırt und verwaltet werden. Die Rechnungsvorschriften bestimmen, daß als Prämienreserve in der Gewinnund Verlustrechnung und in der Bilanz nur die in eigener Verwaltung befindlichen Betrïge geführt werden sollen. Für alle bis zum Inkrafttreten des Gesetzes abgeschlossenen Versicherungen sollen die Prämienreserven unter Berücksichtigung der geltenden Rückversicherungsverträge eingestellt werden. Bei den nach dem Inkrafttreten des Gesetzes abgeschlossenen Versicherungen sollen die Prämienreserven für alle selbst abgeschlossenen Versicherungen einschlieflich der in Rückdeckung gegebenen Summen unter dem Posten Prämienreserve nachgewiesen werden; für die in Rückdeckung ülernommenen Versicherungen aber, soweit die Prämienreserven abzugeben sind, sollen diese Prämienreserven in der Gewinn- und Verlustrechnung bei den Vergütungen für in Räckdeckung übernommene Versicherungen verrechnet werden, also auch in der Bilanz nicht bei den Prämienreserven erscheinen. Die Gesellschaften verfahren bei der Bchandlung 
der Prämienreserven nicht gloıchmäßig. Ein Teil der Anstalten läßt die Prämienreserven in fremder Verwaltung ganz aus der Rechnung fort, während die übrigen Gesellschaften diese Posten bei anderen Stellen behandeln. Da der Vordruck L 3 der Rechnungsvorschriften die Mittel bietet, den Betrag der bei Rückversicherungen abgegebenen Rücklagen fest/ustellen, so sind diese Betrige in unsern Statistılien überall ausgeschieden. Dieses Verfalıren entspricht nicht nur den Rechnungsvorschriften, sondern verhindert auch, daß die Rucklagen für solche Versicherungen, die von einer unter Reichsaufsicht stehenden Gesellschaft hei einer ebensolchen Gescllschaft in Rückdeckung gegeben sind, melirfach aufgefulirt werden.

Die Trennung der Prämienreserven und der Prämienübcrtrïge erfolgt bei den verschiedenen Anstalten und bei verschiedenen Versicherungsarten nicht nach denselben Grundsätzen, so daß die als Prämienreserven oder Prämienibertraige angegebenen Summen weder die reinen Prämicnreserven noch die reinen Ubertrïge darstellen. Pramienreserven und Prämienubertı̈̈ge sind deshalb zusammengefaßt worden.

In Tafel I 39 sind für alle deutschen Aktiengesellschaften Prämienreserven und Prämienüberträge in eigener Verwaltung in Ilohe ron $34.43,331$ Millionen Mark nachgewiesen. Davon kommen 3335,357 Millionen Mark auf die Lebensversicherung und

\begin{tabular}{|c|c|c|c|c|c|c|}
\hline \multirow{3}{*}{ Versicherungsart } & \multicolumn{6}{|c|}{ Prïmenteserven und $P_{1}$ ämenuberträge } \\
\hline & \multicolumn{2}{|c|}{$\begin{array}{l}\text { bet del Gesamtheit } \\
\text { det Untermehtumgen }\end{array}$} & \multicolumn{2}{|c|}{$\begin{array}{l}\text { bet den Altren- } \\
\text { gesellschaften }\end{array}$} & \multicolumn{2}{|c|}{$\begin{array}{l}\text { bei den Gegen- } \\
\text { seltugheitsveieuren }\end{array}$} \\
\hline & $1000 A$ & $1 \%$ & $1010 \mathscr{A l}$ & $1 \%$ & $1000 \mathscr{A}$ & $0^{\prime}$ \\
\hline 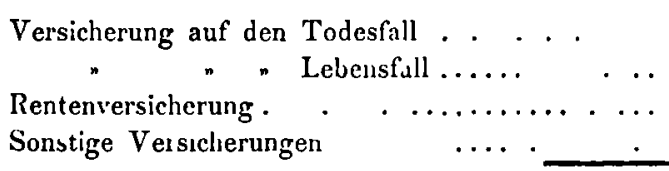 & $\begin{array}{r}4877348 \\
296556 \\
238962 \\
15323 \\
\end{array}$ & 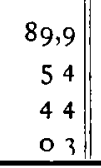 & $\begin{array}{r}2944342 \\
223896 \\
153694 \\
13425 \\
\end{array}$ & $\begin{array}{r}88,3 \\
6,7 \\
4,6 \\
04\end{array}$ & $\begin{array}{r}933006 \\
72660 \\
85268 \\
\text { I } 898 \\
\end{array}$ & $\begin{array}{r}92.3 \\
35 \\
4,1 \\
01\end{array}$ \\
\hline Zusammen ... & $5428+89$ & 100,0 & 3335357 & 100,0 & 2092832 & 100,0 \\
\hline
\end{tabular}

107,974 Millionen Mark auf andere Versicherungøzweige. Bei den Gegenseitigkeitsvereinen gelıören die in der Tafel aufgreführten Betrage von zusammen 2 092,832 Millionen Iark vollständig der Lebensversıcherung an. Die gesamte Primienreserve fiir Lebensund Krankenversicherung stellt sich danach für alle deutschen allgemeinen Unternehmungen auf 5428,189 Millionen Mark, wovon 61,4 v. II. auf die Aktiengesellachaften und 38,6 v. H. auf die großen Gegenseitigkeitsvereine entfallen. In welchem Maße die einzelnen Versicherungsarten betelligt sind, ist aus der Übersicht auf $\mathrm{S}$. $36^{*}$ zu entnehmen.

Die Verteilung des Gewinns ist in Tafel I 40 dargestellt. Wenn dabei die Summe des Gesamtgewinns der Gegenseitigkeitsvereine nicht mit der Summe der Jahresüberschüsse und der Vorträge aus dem Vorjalne, wie sie in der Bilanz ('Tafel I 37) enthalten sind, übereinstimmt, so erkhirt sich das daraus, daß auch die Verteilung des vom Allgemeinen Deutschen Versicherungsverein in Stuttgart aus dem Lebensversicherungsgeschäft erzielten Gewinns nachgewiesen ist, wahrend die Bilanz dieses Vereins bei Gruppe II gegeben ist.

Sonach standen für die Gewinnverteilung zur Verfigung bei den Aktiengesellschaften $108533920 \mathscr{A}$ und bei den Gegenscitıgkentsvereinen $75695722 \mathscr{A}$; diese Beträge sitd folgendermaken verteilt worden: 


\begin{tabular}{|c|c|c|c|c|c|c|c|}
\hline \multirow{9}{*}{ 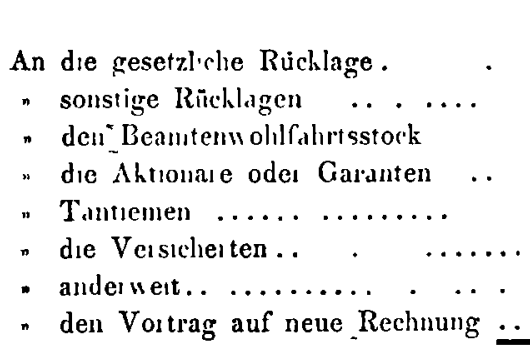 } & \multicolumn{3}{|c|}{27 Ahtiengesellschaften } & \multicolumn{4}{|c|}{18 Gegenseitigkeitsvereine } \\
\hline & 178270 & $\mathscr{M}$ oder & $0,2 \%$ & 620973 & $M$ oder & 0,8 & $\%$ \\
\hline & 8910333 & $n \quad n$ & $8,2 \cdots$ & 6257747 & . " & 8,3 & " \\
\hline & 445817 & $"$ & $0,4 \cdots$ & 327984 & $\cdot$ & 0,4 & . \\
\hline & 8034322 & $"$ & $7,4 n$ & $729 \mathrm{I}$ & - & $(0,009)$ & - \\
\hline & $27395: 5$ & $\cdot$ & $2,5 n$ & I 81722 & $\cdot$ & 0,3 & . \\
\hline & 86532124 & n & 79,7 . & 67302611 & n & 88,9 & . \\
\hline & 508250 & n & 0,5 & $8+7394$ & $n$ & $\mathrm{I}, \mathrm{I}$ & n \\
\hline & $118 ; 289$ & $n$ & I $1 n$ & I50000 & $n$ & $\mathbf{0} 2$ & 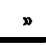 \\
\hline
\end{tabular}

IIinsichtlich der den Versicherten zugewiesenen Beträge ist zu beachten, daß cinige Gesellschalten den den Versicherten zustehenden Gewinn in der Blanz bereits verrechet haben, so dab er lice nicht in die Erscheinung tritt. Diese Betrige (vgl. Fußnoten zu 'Tafel I 40 und IV 40) haben im Berichtsjahre insgesamt 5,846 Millionen Mark ausgemacht.

Die Bilanzen der Versicherungseinrichtungen von Berufsvercinigungen mit den zugehörigen Erliuterungen sind in den Tafeln I 42 bis I 46 gegeben. Die Gesamtbilanz schließt mit $157308516 \mathscr{A}$ ab; die IIauptposten weisen folgende Beträge auf:

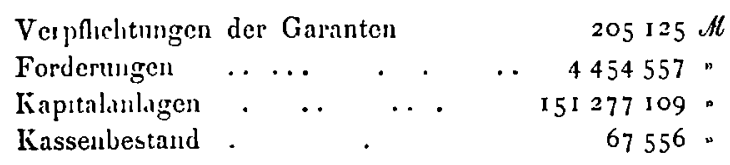

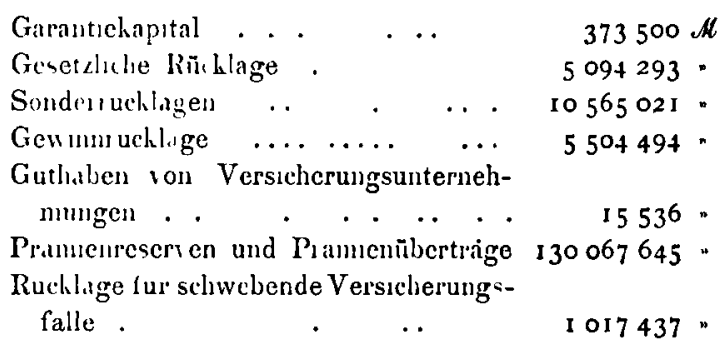

Von den insgesamt $151277109 \mathscr{M}$ betragenden Kapitalanlagen kamen $1232642 \mathscr{M}$ oder 0,8 v. H. auf Grundbesitz, $94246677 \mathscr{L}$ oder 62,3 v. H. anf IJypotheken, $11505146 \mathscr{M}$ oder 7,6 v H. auf Darlehen an offenthehe Korperschaften, 41787259 $\mathscr{H}$ oder 27,6 v. H. auf Wertpapiere und der Rest von $2503016 \mathscr{M}$ oder 1, ז v. II. auf Darlehen auf Versicherungen. Die Vergleichung des Bestandes am Inde des Vorjahrs mit dem Bestand am Ende des Berichtsjahrs ergibt eine Abnahme des IIypothekenbestandes um $1322966 \mathscr{A l}$ und eine Zunalıme des Grundbesitzes um 3 (\$13 $\mathscr{H}$, der Darlehen an öffentliche Körperscląaften um $2651886 \mathscr{M}$, des Buclivertes der Wertpapiere um $10008623 \mathscr{M}$ und der Darlehen auf Versicherungen um $58653 \mathscr{M}$.

Die Bilanzen von 12 Anstalten von Berufsvereinigungen weisen einen Gewinn von zusammen $1932732 \mathscr{N}$ auf, der wie folgt verteilt worden ist: $89334 \mathscr{A l}$ oder 4,6 v. H. an die gesetzliche Rücklage, $631752 \mathscr{N}$ oder 32,7 v. H. an die Sonderrücklagen, $11686 \mathscr{M}$ oder 0,6 v. H. an den Beamtenwohlfahrtsstock, $8423 \mathscr{M}$ oder 0,4 v. II. Tantiemen, $1068533 \mathscr{A}$ oder 55,3 v. II. an die Versicherten, $22320 \mathscr{M}$ oder 1,2 v. II. Vortrag auf neue Reclınung -und $100000 \mathscr{M}$ oder 5,2 v. H. fur anderweite Zwecke.

Die Bilanzen von 17 ausländischen Gesellschaften, deren Lebensversicherungsgeschäfte in dieser Statistik behandelt sind, finden sich in Tafel I 47. Die Bilanzen sind nach Möglichkeit den fur die Blanzen der deutschen Unternehmungen geltenden Grundsätzen angepaßt worden. 
Von besonderem Interesse ist die Art der Kapitalanlagen ('Tafel I 48), die von den Gesellschaften der verschirdenen Länder, sei es aus eigenem Antriebe, sei es auf Veranlassung der Aufsichtshehörden, bevorzugt wird; deslaalb sind in der folgenden Ubersicht die in der 'Tafel I 48 aufgerü̈hrten ausländischen Gesellschaften nach II'imatländern zusammengefaßst und zum Vergleiche die Verlı̈ltniszahlen für die deutsclien Unternelımungen wiederholt:

Von je $1000 \mathscr{M}$ Kapitalanlagen entficlen auf:

\begin{tabular}{|c|c|c|c|c|c|c|c|c|}
\hline & $\begin{array}{l}\text { Grund- } \\
\text { besitz }\end{array}$ & $\begin{array}{l}\text { Iypo- } \\
\text { thichen }\end{array}$ & $\begin{array}{l}\text { Darlehen an } \\
\text { oflentliche } \\
\text { liurper- } \\
\text { scliaften }\end{array}$ & $\begin{array}{c}\text { Darlehen } \\
\text { auf Wert- } \\
\text { papiere }\end{array}$ & $\begin{array}{l}\text { Wert- } \\
\text { paprere }\end{array}$ & $\begin{array}{l}\text { Darlehen } \\
\quad \text { auf } \\
\text { Versiche- } \\
\text { rungen }\end{array}$ & Wechsel & $\begin{array}{c}\text { Gesamthetrag } \\
\text { der liapital- } \\
\text { aulugen } \\
1000 \AA\end{array}$ \\
\hline \multicolumn{9}{|l|}{ bel } \\
\hline 4 amerihanischen Gesellschaften & 29 & 205 & J & $(0,1)$ & 588 & 177 & - & 8505138 \\
\hline 1 dảnuselien Gesellsedraft & 34 & 202 & - & - & 680 & 84 & - & 75549 \\
\hline 2 meder landıst henGesell-chaften & 110 & 282 & - & 5 & $5 \cdot 3$ & 90 & - & 160355 \\
\hline 7 osterrew hischen Gescllschirfen & 99 & $33^{2}$ & 64 & 10 & 396 & 99 & $(0,3)$ & 903861 \\
\hline $\begin{array}{l}3 \text { schweizerischen Gesellschaften } \\
\text { dngegen ber }\end{array}$ & 47 & 695 & 21 & 2 & 170 & 64 & I & 270342 \\
\hline 28 deutschen $\Delta$ htiengescllschaften & 24 & $75^{8}$ & 50 & $(0,1)$ & 89 & 79 & $(0,006)$ & $399882 \mathrm{I}$ \\
\hline * Gegenseitugkeitsi cr- & & & & & & & & \\
\hline cmen.... . & 8 & 729 & 78 & $\mathbf{I}$ & 94 & 89 & I & 2380199 \\
\hline - Berufsı cremigungen & 8 & 623 & 76 & $(0 \quad 0 I)$ & 276 & 17 & - & 151277 \\
\hline
\end{tabular}

Das ausLindische Geschaft der deutschen Unternelhmungen

Von den allgemeinen deutschen Unternchmungen haben die Kapitalversicherung in den wichtigeren Staaten des Auslandes mittelbar oder unmittelbar betricben: 23 in der Schweiz, 22 in Österreich, 21 in Dänemark, 18 in Belgien, 17 in den Niederlanden, 9 in Norwegen, 8 in Frankrcich, 7 in der Türkei, je 6 in Schweden, Luxemburg, Rußland und Spanien, je 5 in Ungarn und Ägypten, je 4 in China, England, Finnland, Portugal, Rumänien und in den Vereinigten Staten von Amerika, je $\mathbf{3}$ in Argentinien und in Brasilien, 2 in Bolivien, je 1 in Bulgarien, Chile, Griechenland und in Japan. In der Rentenversicherung ist das Geschäft natürlich auch im Auslande weniger verbreitet; es haben gearbeitet: 12 Unternehmungen in der Schweiz, 11 in Belgien, 9 in den Niederlanden, 8 in Österreich, 7 in Dämemark, je 5 in Frankreich und in Lưxemburg, 4 in Rußland, je 3 in England, Japan, Norwegen, Schweden, Türkei und in den Vereinigten Staaten von Amerika, je 2 in Ägypten, Brasilien, Spanien, Ungarn und Rumänien, je 1 in Argentinien, Chile, China, Finnland, Griechenland, Mexiko und Portugal.

In der Kapitalversicherung hatten die Unternehmungen zusammen Ende 1916 im Auslande 291584 laufende Verträge uber 1561,740 Millionen Mark, das sind 2,2 v. H. aller Versicherungen und 9, $8 \mathrm{v}$. H. der gesamten Versicherungssummen; die vercinnahmten Beiträge, einschlicßlich der für Rückversicherungen wieder abgegebenen Beiträge betrugen 69,8(13 Millionen Mark, wovon 67,017 Millionen oder 96,0 v. H. für selbst abgeschlossene und 2,786 Millionen oder 4,0 v. II. für in Rückdeckung übernommene Versicherungen zu rechnen sind. An Versicherungsleistungen wurden 31,149 Millionen Mark gewährt, nämlich 29,415 Millionen für selbst algeschlossene Versicherungen und 1,734 Millionen für Rückversicherungen; von den in der ausländischen Kapitalversicherung überlıupt fällig gewordenen Beträgen kommen demnach 5,6 v. H. auf die in Rückdeckung übernommenen Versicherungen. 
In der Rentenversicherung liefen Ende 1916 im Auslande 3913 Versicherungen über 1,719 Millionen Mark Jalıresiente, das sind 5,5 v. II. aller Versicherungsschicine und 5,6 v. II. der gesamten Jahresrente. An Beiträgen wurden vercinnalimt 0,253 Millionen Mark. Für Versicherungsleistungen waren 1,108 Millionen Mark bereitzustellen.

Zu ciner cingehenden Vergleichung des ausländischen Gesclıäfts aller deutschen Lcbensversicherungs-Unternchmungen mit dem deutschen Geschäfte der ausländischen Gescllschaften fehlt der Stoff, doch bicten folgende Zahlen wenigstens einige Anhaltspunkte:

Im Jahre 1916 hat die Beitragseinnahme betragen:

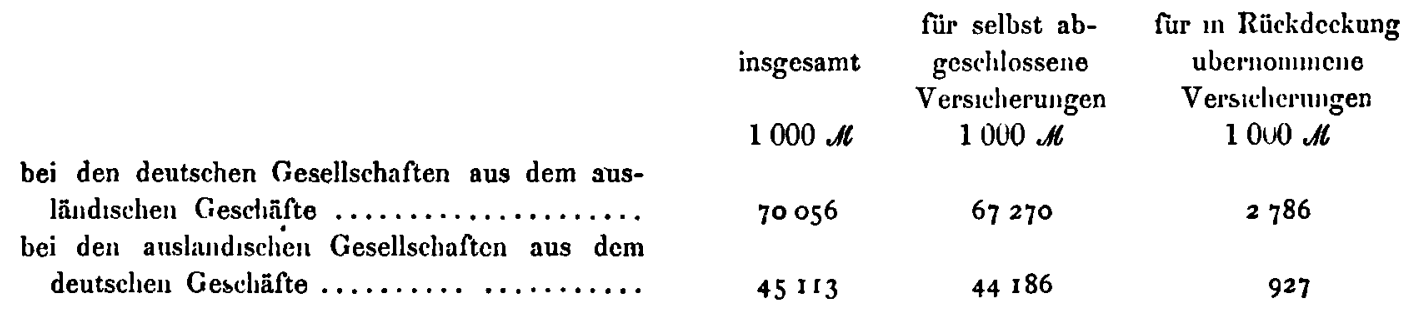

Hirrnach haben insgesamt die deutschen Gesellschaften für Lebensversicherungen 24,043 Millionen Mark melır an Beiträgen aus dem Auslande als die hier berücksichtigten ausländischen Gesellschaften aus dem Deutschen Reiche eingenommen. Die Zahlungen an Versicherungsleistungen haben betragen:

\begin{tabular}{|c|c|c|c|}
\hline & insgesamt & $\begin{array}{l}\text { für sclbst ab- } \\
\text { geschlossene } \\
\text { Versichorungen }\end{array}$ & $\begin{array}{c}\text { für in Rïckdechung } \\
\text { ühernommene } \\
\text { Verstehet ungen }\end{array}$ \\
\hline ei den deutschen Gesellschaften für auslandische & $1000 \mathscr{A}$ & $1000 \mathscr{A}$ & $1000 \mathscr{H}$ \\
\hline $\begin{array}{l}\text { Versicherungen } \ldots \ldots \ldots \ldots \ldots \ldots \ldots \ldots \ldots \\
\text { ei den ansländischen Gesellschuften für deutsche }\end{array}$ & 32257 & 30523 & I 734 \\
\hline Versicherungen $\ldots \ldots \ldots \ldots \ldots \ldots \ldots \ldots$ & 36825 & $3618 \mathrm{r}$ & 644 \\
\hline
\end{tabular}

Nälıcres hierüber findet man in der „Entwickelung " S. 13.

Außer den besprochenen und in den Tafeln behandelten deutsclien Versiche- Die klenen rungsunternehmungen steht, wie bereits erwïlint, gemïß $\$ 2$ V.A.G. noch eine Anzalıl Punsunskleiner Sterbe- und Pensionskassen unter Reichssaufsicht, die für eng begrenzte Kreise kassen bestimmt und vielfach ron gewerblichen Firmen nur für die eigenen $\Lambda$ ngestellten oder Arbeiter eingerichtet sind. Die Zahl dieser Gegenseitigkeitsvereine ist schwankend; einerseits sind diese Unternehmungen wohl kaum sämtlich bekannt, auch entstehen stets neue derartige Vereine; anderscits gelht die Beaufsichtigung nicht selten gemäß § 3 Abs. 2 nachträglich an die Landesbehörde über. Andere Vereine verfallen der Auflösung. Wie dem auch sci, die Zahl der unter Reichsaufsicht stehenden Stcrhokassen und lensionskassen wird immer nur sehr klein scin im Vergleiche zu der Zahl der unter Landesaufsicht befindlichen; wenn hier einige Mitteilungen über die unter unserer Aufsicht stehenden Kassen gemacht werden, so können daraus keineswegs Schlüsse über die Bedeutung des Sterbekassen- und Pensionskassenwesens im Reiche gezogen werden.

Für das Jahr 1916 haben insgesamt 133 solcher Sterbekassen dem Aufsichtsamte befriedigende Jahresberichte eingereicht. Danach ist die Zahl der Mitglieder 
Ende 1916 auf rund 514947 anzusetzen; die Finnahmen haben im Berichtsjahre $9121307 \mathscr{M}$ betragen, die Ausgaben $541,9287 \mathscr{H}$.

Von den Einnalımen kommen $6774239 \mathscr{A}$ orler 74,2 v. H. auf Beiträge für die Mitglieder, $54288 \mathscr{M}$ oder 0,6 v. II. auf Eintrittsgelder, $2247288 \mathscr{H}$ oder 24,6 v. H. auf Vermögenserträge und $4 ; 4 ! 92 \mathscr{H}$ oder 0,6 v. $H$. auf andere Einnahmen Von den Ausgaben kommen $3236968 \mathscr{H}$ oller 59,2 v. H. auf Sterbegelder, $739655 \mathscr{A l}$ oder 13,5 v. H. auf Krankengelder, $41015 \mathscr{A}$ oder 0,7 v. H. auf Zahlungen für vorzeitig aufgelöste Versicherungen, $358980 \mathscr{A}$ oder 6,6 v. H. auf Gewinnanteile, $551642 \mathscr{M}$ oder 10,1 v. II. auf Verwaltungskosten und Steuern und $541027 \mathscr{A l}$ oder 9,9 v. II. auf die übrigen Ausgalien. Das Vermögen dieser Kassen belief sich am Schlusse des Jalıres 1916 auf $54440399 \mathscr{M}$.

Die Zahl der Pensionskassen der genannten Art, die in befriedigender Weise über ihren Geschäftshetricb im Jahre 1916 an das $\Lambda \mathrm{mt}$ berichtet haben, betrug 90; diese Kassen hatten Ende I9 5113182 aktive Mitgheder und an Bezugsberechtigten 5882 Pensionierte, 3424 Witwen und 1561 Waisen und Ende $1916113838 \mathrm{Al}$ live, 58.59 Pensionierte, 3729 Witwen und 1 dis6 Waisen Die Einnahunen betrugen im Berichtsjahre $33398963 \mathscr{A}$, wahrend $13694236 \mathscr{H}$ verausgabt wurden Von den Einnahmen entfallen $19152743 \mathscr{H}$ oder 57,4 v. H. auf Beiträge für die Mitglieder, $138968 \mathscr{N}$ oder 0,4 v. II. auf Eintrittsgelder, $10396400 \mathscr{H}$ oder 31,1 v. II. auf Vermögenserträge und $3710852 \mathscr{N}$ odler 11,1 v. II. auf anderweitige Einnalımen. Von den Ausgaben entfallen $6243297 \mathscr{A}$ oder 45,6 v. II. auf Pensionen, $145400 \mathscr{M}$ oder 1,1 v. H. auf Krankengeld, 577 10!) $\mathscr{A}$ oder 4,2 v. H. auf Sterbegeld und sonstige Leistungen an Versicherte, $558821 \mathscr{N}$ oder 4,1 v. II. auf Abfindungen beim Austritt aus der Kasse (meist infolge Wechsels der Dienststelle), $610 j 5 \mathscr{A l}$ oder 0,4 v. II. auf Gewinnanteile, $389544 \mathscr{A}$ oder 2,8 v. II. auf Verwaltungskosten und Steuern und $5719002 \mathscr{M}$ oder 41,8 v. II auf die ibrigen Ausgaben. Der niedrige Satz für Verwaltungskosten hat seinen Grund in erster Linie darin, daß bei den von gewerblichen Firmen eingerichteten Versicherungskassen die Verwaltungskosten meistens vollständig von den Firmen ubernommen werden und somit die Kassen nicht belasten. Das Vermögen dieser Pensionskassen belief sich am Ende des Berichtsjahrs auf $240279882 \mathscr{M}$.

\section{Gruppe II, Unfall- und Haftpflichtversicherung}

Die Unternehnungen

Von den großen deutschen Versicherungsunternehmungen, welche der Reichsaufsicht unterstehen, waren im Jahre I9 633 auf dem Gebiete der Unfall- und Haftpflichtversicherung tätig, 32 Aktiengesellschaften und 1 Versicherungsverein auf Gegenseitigkeit. Die Anzahl der im Berichtsjalıre zu behandelnden Gesellschaften hat sich um eine vermelırt, indem die IIohenzollern, Versicherungs-Aktiengesellschaft in Cöln, die jetzt auch die Unfall- und die Haftpllichtversicherung betreibt, hinzugekommen ist.

Außerdem berichtet die vorliegende Statistik wieder übcr acht ausländische Gesellschaften, dieselben, die in der vorjälırigen Statistik behandelt sind. 
Zur Beurteilung des Umfanges des Geschäfts der deutschen Gesellschaften soll die Tafel II 1 dienen. Daraus ist zu ersehen, daß bei diesen Unternelımungen am Ende des Jalıres 1916 in der Unfallversicherung 1273295 und in der Haftpllichtversicherung 269 i 6 (i5 Versicherungsscheine über selbst abgeschlossene oder in Rückdeckung übernommene Versiclierungen in Kraft waren.

In der IIaftpflichtversicherung wurden Versicherungen mit Gewinnbeteiligung außer von dem Allgemeinen Deutschen Versicherungs-Verein in Stuttgart nur von der Oberrheinischen Versicherungs-Gesellschaft in Mlannheim, und zwar nur in ganz geringem Umfang abgeschlossen. Auch in der Unfallversicherung kommen Versicherungen mit Gewinnbeteiligung nur bei wenigen Unternehmungen in Betracht. Von den Ahtiengesellschaften haben nur zwei solche Versicherungen abgeschlossen, darunter eine auch nur in ganz geringem Umfange. Von der Beitragseinnahme des Geschäftsjahrs in Ilöhe von $33312629 \mathscr{A l}$ für selbst abgeschlossene Unfallversicherungen sämtlicher Unternelımungen kamen auf'Versicherungen mit Gewinnbeteiligung nur $9274479 \mathscr{M}$ oder 27,8 v. H., oder, wenn man den Allgemeinen Deutschen Versicherungs-Vercin in Stuttgart, weil er eine Gewinnbetciligung nur auf Grund seines Charakters als Gegenseitigkeitsverein kennt, ausscheidet, 16,9 v. H.

In den fuir die Unfallversicherung gegebenen Zahlen ist auch die Zeitungsabonnentenversicherung enthalten, die von 2 Gesellschaften nämlich der Deutschland, Lcbensversicherungs-Aktien-Gescllschaft zu Borlin, und der Nürnberger LebensversicherungsBank. betrieben wird. Iie Anzahl der hierdurch versicherten Personen beläuft sich auf rund 2310000 mit $2305000 \mathscr{A l}$ Beitragseinnalıme.

Für das Rückversicherungৎgeschäft lassen sich folgende Zahlen angeben. In der Unfallversicherung waren in Rückdeckung übernommen für $1994816 \mathscr{M}$ Beiträge oder 5,6 v. II. der ganæen Beitragseinnahme von $35307445 \mathscr{M}$ und in Rückdeckung gegeben für $7152659 \mathscr{A}$ oder für 20,2 v. II. der ganzen Beitragseinnalıme. In der Haftpllichtverșicherung waren in Rückdeckung übernommen für $1847952 \mathscr{M}$ Beiträge oder 3,8 v. H. und in Rückdeckung gegeben für $11288996 \mathscr{N}$ oder 23,5 v. H. der gesamten Beitragseinnalıme von 4805 j $092 \mathcal{M}$.

In diesen wie in allen folgenden Zahlen sind beim Allgemeinen Deutschen Versicherungs-Verein in Stuttgart in der Haftpflichtversicherung auch die auf die Versicherung gegen Wasserleitungsschäden und einige andere kleine Sachversicherungszweige bezüglichen Zahılen enthalten.

Vergleicht man den Versicherungsbestand am Ende des Berichtsjahrs mit dem entsprechenden Bestand am Ende des Vorjalırs ('Tafel II 2), so findet man, daß die Zahl der Versicherungsscheine bei der Unfallversicherung um 57487 oder 4,7 v. H. zugenommen und bei der Haftpllichtversicherung um 27260 oder 1,0 v. H. des Bestandes am Encle des Vorjalırs alogenommen hat. Die gesamte Beitragseinnahme hat im Geschäftsjahre bei der Unfallversicherung $1787281 \mathscr{M}$ oder 4,8 v. H. und bei der Haftptlichtversicherung $410170 \mathscr{H}$ oder 0,8 v. H. weniger als im Vorjahre (bei der Unfallversicherung $12320030 \mathscr{M}$ oder $24.9 \mathrm{v} H$. und bei der Haftplichtversicherung $9441803 \mathscr{N}$ oder 16,8 v. H.) betragen.

Es ist also auch in diesem $\mathrm{Jahr}$ ein weiterer, wenn auch unerheblicher Rückgang in der Beitragseinnahme als Folge des Krieges zu verzeichnen. In der Friedenszeit 
hatte die Finnahme von Jahr zu Jahr zugenommen und selbst die mit der Durchführung der weitgehenden 'Tarifreform vom Jahre 1904 verbundenen Schwierigkeiten im Geschäftsbetriebe hatten I905 wohl ein Stocken im Anwachsen, aber doch keinen Rückgang der Beitragseinnahme zur Folge.

Die

Betrebs-

Einnalimen und

-Ausgaben

des

Berichts-

jalirs

In unseren Statistiken sind von vornherein die den einzelnen Versicherungszweigen eigentümlichen und in den Büchern getrennt behandelten Posten einzeln aufgeführt worden; seit 1905 konnten auch die allgemeinen Verwaltungskosten und die Steuern mit befriedigender Genauigkeit nach Geschäftszweigen getrennt werden. Seitdem ist der Gewinn oder Verlust aus dem eigentlichen Versicherungsbetriebe des Geschäftsjahrs bei jedem Zweige ermittelt worden; Einnalımen und Ausgaben, die niclit unmittelbar mit dem Versicherungsbetriebe des Berichtsjahrs zusammenhängen, wie z. B. bei der Sachversicherung die Erträge der Vermögensverwaltung, bleiben dabei außer Betracht (vgl. "Lintwickelung " S. 33). Bei der Unfall- und IIaftpflichtversicherung ist indessen der Betricbs-Gewinn oder -Verlust nicht vollstïndig ermittelt worden, weil der Frtrag der die Prämienreserve deckenden Kiapitalanlagen, der zweifellos zu den Betriebs-Finnalimen zu rechnen ist, nicht sicher angegeben werden kann. Schätzungen des Amts sollen aber in dieser Statistik vermieden werlen. Um alle Mißverstänlnisse auszuscliließen, ist in 'Tafel II $3 \mathrm{kr}$ in Gewinn oder Verlust eingestellt. Das Frgelonis der Vermögensverwaltung (vgl. S. 43*) findet sich in Tafel II 5. Dcr Gewinn oder Verlust des alle Zweige umfassenden Gesamtgeschäfts wird in der Bilanz ausgewiesen.

Als Betriebs-Finnalımen sind bei Gruppe II verrechnet die Beiträge für das Geschäftsjahı, d.h. die Beiträge unter Berüclisichtigung der Uberträge aus dem Vorjahr und der Clherträge auf das folgende Jahr, einschließlich der an die Rückiversicherungsgesellschaften gezahlten Rückversicherungshciträge, ferner die Ausfertigungsgel,ühren, die Vergütungen der Rückversicherer, der Unterschicd zwischen der Schadenrücklage aus dem Vorjahr und den Zahlungen und Rückstellungen für Versicherungsfälle der Vorjahre aus scllıst abgeschlossenen Versicherungen, sofern die Schadenrücklıge größer ist, und der etwaige Mehrbetrag der aus dem Vorjahr übernommenen Prämienreserve gegenüber der am Schlusse des Jahres zu stellenden Prämienreserve. Die Betriebs-Ausgalıen setzen sich bei Gruppe II zusammen aus den Zahlungen für die selbst abgeschlossenen und für die in Rückdeckung übernommenen Versicherungen einschließlich des Anteils der Rückversicherer, aus den Rückversicherungsbeiträgen, dem Untcrschiede zwischen der Schadenrücklage aus dem Vorjahr und den Zahlungen und Rückstellungen für Versicherungsfälle der Vorjahre aus selbst abgeschlossenen Versicherungen, sofern die Schadenrücklage kleiner ist, und endlich aus der Zunahme der Prämienreserve. Es soll hier aber besonders betont werden, daß der Unterschied zwischen der Schadenrücklage aus dem Vorjahr und den Zahlungen und Rückstellungen für Versicherungsfälle aus selbst abgeschlossenen Versicherungen der Vorjahre allein keinen Schluß darüber zuläßt, ob die Schadenrücklage zulänglich bemessen war. Denn einerseits sind aus der Schadenrücklage auch andere Leistungen als Zalilungen für Versicherungsfälle der Vorjahre zu bewirken, z. B. Zahlungen für die in Rückdeckung übernommenen Versicherungen und Zuführungen zur Prämienreserve. Anderseits wird die Schadenrüclilage meist olne den Anteil der Rückversicherer eingestellt, wogegen die Zahlungen einschließlich dieses Anteils erscheinen. Auch enthalten die 
Zahlungen für Versicherungsfälle der Vorjahre Betrige, die nicht aus der Schadenrücklage, sondern aus der Prämienreserve zu decken sind.

Die Betriebs-Finnahmen des Berichtsjahrs haben olıne die Frträge der Prämienreserven insgesamt bei den 33 deutschen Gesellschaften 109478492 Ml betragen; davon kamen auf Beitrïge für Unfallversicherung $35017518 \mathscr{A l}$ oder $32,0 \mathrm{v}$. II., auf Beiträge für Iaftpflichtversicherung $47508230 \mathscr{M}$ oder 43,4 v. $H$. und auf Vergütungen der Rückversicherer $15231846 \mathscr{M}$ oder 13,9 v. II. aller dieser Betriebs-Einnahmen.

Die Summe der Betriels-Ausgaben ohne Verwaltungskosten beziffert sich auf $70003028 \mathscr{H}$; davon entfillen 4210160 J $\mathscr{M}$ auf Zahlıngen für Versicherungsfälle im Geschäftsjahr aus selbst abgeschlossenen Versicherungen, und zwar (Tafel II 4) $142.99066 \mathscr{A}$ oder 20,4 v. H. aller Betriebs-Ausgaben auf Unfallversicherungsfälle, ferner $22313406 \mathscr{N}$ odler 31,9 v. II. auf Ilaftpflichtrersicherungsfialle, $1999444 \mathscr{A}$ oder 2,9 v. H. auf laufende Renten und endlich $3529689 \mathscr{A}$ oder 5,0 v. II. auf Beitragsrückgewälırbeträge. Von der Summe der Betriebs-Ausgaben entfallen ferner $6519103 \mathscr{N}$ oder 9,3 v. H. auf Vergütungen für die in Rückdeckung übernommenen Versicherungen (darunter $2252939 \mathscr{A}$ oder 3,2 v. H. für Unfallversicherungsfalle und $2963226 \mathscr{A l}$ oder 4,2 v. H. für IIaftyflıchtversichıerungsfälle), $7152659 \mathscr{H}$ oder 10,2 v. II. auf Rückversicherungsbeiträge für Unfallversicherungen und $11288996 \mathscr{M}$ oder 16,1 v. H. auf Rückversicherungsbeiträge für IIaftpflichtversicherungen.

Die 33 Gesellschaften haben an Verwaltungskosten und Steucrn insgesamt $30380778 \mathscr{M}$ für das Unfall- und IIaftpflichtversicherungsgeschäft aufgewandt.

Wenn man annimmt, daß die Vergütungen für die in Rückdeckung übernommenen Versicherungen sich nur auf Versichermngsfälle des Geschüftsjahıs beziehen und daron absielıt, daß ein 'Teil der laufenden Renten, die ja in ihrer Gesamtheit nur geringe Betrïge erfordern, aus früheren Jahren herrührt, so kann man den Gesamthetrag der Schadenzahlungen (49014377 $\mathscr{C}$ ) in Bezichung setzen zur Beitragscinnahme fiir das Geschiiftsjahr (S2 525 748 $\mathscr{A}$ ). Man findet dann, daß von je $1000 \mathscr{A l}$ Beitragscinnahme $594 \mathscr{N}$ für Schadenzahlungen verausgal)t wurden; weitere $22 \mathscr{N}$ wurden zur Hihóhung der Prämienreserven verwandt. 'Tatsächlich ist allerdings für eigentliche Scliảen etwas weniger aufgewandt worden; denn cinzelne Gesellschaften verrechnen bei den Schadenzahlen auch noch mancherlei andere Beträge, -z. B. Schadenermittelungskosten. -- In den letzten fünf Jahren haben diese Zahlen betragen:

\begin{tabular}{|c|c|c|c|c|c|}
\hline & 1912 & 1913 & I 9 I 4 & 1915 & 1916 \\
\hline $\operatorname{gen} \ldots \ldots \ldots \ldots$ & $496 \mathscr{M}$ & $5^{26} \mathscr{M}$ & $519 \mathscr{M}$ & $569 A$ & $594 \mathscr{M}$ \\
\hline bei den Zufuhrungen zur Prämenreserve... & 39. & $43^{\prime \prime}$ & 39 n & $31 \%$ & $22 n$ \\
\hline
\end{tabular}

Das Ergebnis der Vermögensverwaltung wird von den Unternehmungen in der Regel nicht nach den einzelnen Geschäftszweigen getrennt; nur der Lebensversiclıerung werden immer bestimmte Erträge überwiesen, weil das der Betrieb dieses Geschäfts erfordert. Es besteht keine Veranlassung, in dieser Statistik von dem Verfahren der Gesellschaften abzugehen. Soweit also nicht ein Teil des Ertrags einem bestimmten Zweige zugewiesen ist, wird das Ergebnis zumeist nur insgesamt aufgeführt und bei derjenigen Gruppe mitgeteilt, zu der das Hauptgeschäft der Unternelımung gehört. 
Im einzelnen geben die Fußnoten der Tafeln überall da Auskunft, wo nur ein Teil der Vermögensverwaltung in Betracht genommen worden ist.

Unter den Erträgen stehen wie überall so auch hier die Zinsen im Vordergrund, nämlich mit $12969177 \mathscr{N}$; der Kursgewinn bleibt um $384678 \mathscr{M}$ hinter dem Kursverluste zurück, der sonstige Gewinn betr:ïgt $469086 \mathscr{M}$, der sonstige Verlust 123858 $\mathscr{M}$. Als reiner Ertrag sind $13516898 \mathscr{M}$ zu verzeichnen.

Eine Bilanz ist in dieser Statistik grundsätzlich nur für das Gesamtgeschäft einer jeden Gesellschaft gegeben und bei derjenigen Gruppe aufgeführt, zu der das Unternelımen seinem IIaupthetriebe nach - Transportversicherung immer ausgeschlossen gehört So bleiben bei Gruppe II von den 33 deutschen Gesellschaften, welche Unfall- oder Haftpfliclıtversicherungen unmittelbar abschlıeßen, nur 8 Anstalten übrig. Die Bılanzen der Berlinischen Lehens-Versicherungs-Gesellschaft, von Deutschland, Victoria, Urania, Providentia, Janus, Teutonia, Atlas, Magdeburger Lebens-Versicherungs-Gesellschaft, Wilhelma, Bayerische Versicherungs-Bank, Nürnberger Lebensversicherungs-Bank und Germania finden sich bei Gruppe I (Tafel I 37), die Blanzen von Victoria Feuer-Versicherungs-Aktien-Gescllschaft, IIohenzollern, Vaterländische und Rhenania. 'Thuringia, Iduna F.U.H. u. R.V.A.G., Albingia, Globus, Hamburg-Bremer, Rheinland, Prcußısche National V.G. und Stuttgart-Berliner V.A.G. bei Gruppe IV ('Tafel IV 38) und von Hammonia bei Gruppe V (Tafel V 9).

Aus den in Tafel II 6 für die 8 Gesellschaften gegebenen Bilanzen mögen hicr die folgenden Schlußzahlen Platz finden:

Verpfichtungen der Aktionare .
Kapitalanlagen ...
Forderuugen . . .
Kassenbestand

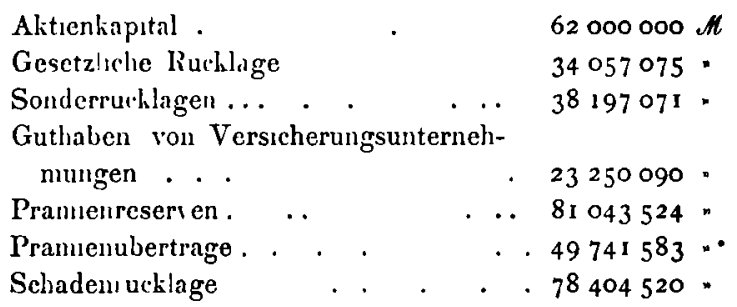

Dabei ist zu erwăhnen, daß aus den Nebenbetrieben der Gesellschaften von den Prämienreserven $63414347 \mathscr{M}$ auf Lebensversicherungen und von den Prämienüberträgen $28198355 \mathscr{A}$ auf nicht zur Gruppe II gelıörige Versicherungszweige entfallen; für die Unfall- und Haftpflichtversicherung bleiben danach an Prämienreserven nur $17629177 \mathscr{N}$ und an Uberträgen $21543228 \mathscr{H}$.

Die Kapitalanlagen $(295884123 \mathscr{M})$ verteilen sich auf folgende Wertarten: $23673163 \mathscr{H}$ oder 8,0 v. H. Grundbesitz, $141170916 \mathscr{H}$ oder 47,7 v. H. IIypotheken, $44099927 \mathscr{A}$ oder 14,9 v. H. Darlehen an öffentliche Körperschaften, $666661 \mathscr{A}$ oder 0,3 v. H. auf Darlehen auf Wertpapiere, 82024 (j09 $\mathscr{H}$ oder 27,7 v. H. Wertpapiere, $4153693 \mathscr{N}$ oder 1,4 v. II. Darlehen auf Versicherungen und $95154 \mathscr{H}$ oder $0, \cap 3$ v. H. Wechsel. Stellt man diese Zahlen denen des Vorjahrs gegenüber, so haben die Darlehen an öffentliche Körperschaften um $15277384 \mathscr{N}$, die Darlehen auf Wertpapiere um $92729 \mathscr{M}$, die Wertpapiere um $8088009 \mathscr{N}$ (nach dem Nennwert um $9947206 \mathscr{N}$ ), die Darlehen auf Versicherungen um $293855 \mathscr{A}$ zugenommen; dagegen haben der Grundbesitz um $453130 \mathscr{H}$, der Besitz an Hypotheken um $1790880 \mathscr{N}$ und die Wechsel IIm $18717 \mathscr{M}$ abgenommen. Sieht man von den Darlehen auf Versicherungen, die den 
öffentlichen Geldverkehr nicht unmittelbar berühren, ab, so haben jene 8 Gesellschaften dem Kipitalmarkte $21195395 \mathscr{M}$ zugeführt.

Jedle der 8 Gesellschaften hat mit einem Gewinn abgeschlossen, der sich für alle Gesellschaften zusammen nach der Bilanz auf $19876362 \mathscr{N}$ und mit Einschluß des Gewinnvortrags aus dem Vorjalır auf $22134465 \mathscr{M}$ stellt gegen $19213020 \mathscr{A}$ und $21397650 \mathscr{U}$ im Vorjahre. Von dem Gewinne des Berichtsjahrs entfallen auf die Lebensversicherungsabteilung des Allgemeinen Deutschen Versicherungs-Vereins in Stuttgart $3736104 \mathscr{N}$, dessen Vertcilung aus Tafel I $40 \mathrm{zu}$ ersehen ist. Von dem Reste, $18398361 \mathscr{H}$, sind nach 'Tafel II 8 überwiesen $6764 \mathscr{N}(0,04 \mathrm{v}$. H.) an die gesetzliche Rücklage, $641286 \mathscr{M}(3,5$ v. H.) an sonstige Rücklagen, $496497 \mathscr{M}(2,7$ v. H.) an den Icamtenwolifahrtsstock, $6216890 \mathscr{A}$ (33,8 v. II.) an die Aktionäre; ferner sind zugeteilt $1706183 \mathscr{M}$ (9,3 v. H.) an Tantiemen, $5706060 \mathscr{M}$ (31,0 v. II.) Gewinnanteile an die Versicherten sowie $1192065 \mathscr{N}(6,5$ v. II.) anderweit; endlich sind $2432616 \mathscr{M}$ $(13,2$ v. IH.) auf neue Rechnung vorgetragen.

In der diesjtilırigen Bilanz stehen die gesetzlichen Rücklagen um $6859 \mathscr{M}$ und die Sonderrücklagen um $803012 \mathscr{A}$ hỏher als in der Bılanz für Ende I9 I5.

Von den hier behandelten 33 deutschen Untcrnehmungen haben 18 Anstalten auch melir oder weniger umfangreiche Unfall- und II:ftphichtversicherungsgeschäfte im Auslande abgeschlossen, nämlich in Bulgarien, Finnland, Japan und den Vereinigten Staaten von Amerika je 1, in Italien, Portugal und Rumänien je 2, in Spanien, der Türkei und Ungarn je 3, in England, Frankreich und Rußland je 4, in Luxemburg und Norwegen je 5, in Schweden 7, in Belgien 10, in der Schweiz 11, in Dänemark 12, in den Niederlanden 13 und endlich in Österreich 14.

Diese Unternchmungen liatten im Auslande 161941 Versicherungen in Kraft, das sind 4,1 v. II. aller Versicherungen. Die vereinnalimten Beitrage, einschlieblich der fuir Rückversicherungen wieder abgegebenen Teile, betrugen für das auslindische Geschäft insgesamt 7,573 Millionen Mark, wovon 6,86s Millionen Mark für die selbst abgeschlossenen und 0,705 Millionen Mark für die in Rückdeckung übernommenen Versicherungen zu rechnen sind; im Verhàltnisse zum Gesamtgeschäfte sind das 9,1 v. H., 8,6 v. H. und 18,3 v. H. Beim auslaindischen Geschäfte machten die Beiträge für das in Rückdeckung übernommene Geschäft 9,3 r. H. von den Beitrïgen für das ganze auslindische Geschält aus. Für Versicherungsleistungen wurden 3,587 Mllionen Mark gewährt, nämlich 2.238 Millionen Mark für selbst abgeschlossene Versicherungen und 1,349 Mlllionen Nark für Rückversicherungen; von der Gesamtausgabe für ausländische Schü̈len kommen demnach 37,6 v. H. auf in Rückdeckung übernommene Versicherungen. Auf $1000 \mathscr{H}$ Beitragseinnalıme kommen $474 \mathscr{M}$ Schadenzahlungen.

Außer den erwähnten großen Unternehmungen unterlagen der Reichsaufsicht noch einige Gegenseitigkeitsvereine und einige Eingetragene Genossenschaften m. b. H. von geringerer Bedeutung, die ihre Mitgheder gegen Haftpflicht oder auch gegen Unfall und Haftpflicht versichern. Eine Anderung gegenuber dem Vorjahre hat nicht stattgefunden, so daß hier wieder die IIauptergebnisse von 11 solelıen Vereinen mitgeteilt werden können.

Die Zahll der Versicherungsscheine dieser 11 Unternehmungen belief sich Ende I 9 I 6 auf 70481 . Die Beiträge für das Geschäftsjahr betrugen für alle Unternehmungen zu- 
sammen $775592 \mathscr{M}$. Davon wurden für Rückversicherungen $143421 \mathscr{M}$ wieder abgefülırt. Verausgabt wurden für Schïden $491264 \mathscr{A l}$ und für Verwaltungskosten $158510 \mathscr{M}$. Das Geschäftsjahr hat für 8 Vereine einen Gewinn von insgesamt $205593 \mathscr{M}$, für zwei Vereine einen Verlust von zusammen $13152 \mathscr{M}$ gebracht; ein Vercin hat die Ausgaben gerale durch die Emnahmen gedeckt. Von dem Gewinne sind $69738 \mathscr{H}$ den gesetzlichen Rücklagen, $23750 \mathscr{A}$ den sonstigen Rüchlagen und $345 \mathscr{M}$ den Garanten überwiesen worden; ferner sind $6100 \mathscr{N}$ an Tantiemen ausgrgeluen, $99424 \mathscr{A}$ den Versicherten gutgeschrieben, $3250 \mathscr{N}$ anderweit verwendet und $2986 \mathscr{A l}$ auf neue Reclmung vorgetragen.

Die Bilanz dieser Unternelımungen schließt mit $4312625 \mathscr{M}$ ab. Als IIauntposten der Vermögenswerte sind zu vermerken $3583912 \mathscr{M}$ Kapitalanlagen, $449423 \mathscr{M}$ Forderungen und $15719 \mathscr{M}$ Kassenbestand; als IInuptposten der Verbindlichkeiten kommen in Betracht $542850 \mathscr{M}$ Bürgsclaftskapital, $2201164 \mathscr{M}$ gesetzliche Rücklagen, $216003 \mathscr{N}$ Prämienüberträge und $871796 \mathscr{N}$ Schadenrücklage.

Zur Erläuterung des Umfanges des deutschen Geschäfts der ausländischen Unternehmungen in der Unfall- und IIaftptlichtrersicherung sollen die Tafeln II 10 bis 12 dienen. Es handelt sich wie früher nur um österreichische, ungarische und schweizerische Gesellschaften.

Die Basler Lebens-Versicherungs-Gesellschaft hat im Deutschen Reiche nur in der Unfillversicherung und in der Lebénsversicherung Geschïile betrieben, während die Fonciere nur in der Unfallversicherung und in der 'Transportversicherung gearbestet hat. Von den anderen Gesellschaften betrieben in Deutschland neben der Unfill- und IIaftpflichtversicherung dic Zürich auch die Einbruchdiebstahl- und die Kautionsversicherung, die Schweizerische National-Versicherungs-Gescllschaft auch die Einbruchıliebstahl-, die Glasversicherung und die Transportversicherung, die Schweizerische UnfallversicherungsAktrengesellschaft in Winterthur die Einbruchliebstahlversicherung und die Kautionsund Bürgschaftsversicherung, die Providentia in Wien die Maschinenversicherung und die Erste Österreichische Allgemeine Unfall-Versicherungs-Gesellschaft in Wien die Wasserleitungsschäden- und Einbruchdiebstahlversicherung.

Acht ausländische Gesellschaften haben aus ihrem Unfallversicherungsgeschïft im Deutschen Reiche $19166042918 \mathscr{M}$ Beiträgge für selbst alggeschlossene Versichırungen und $1898 \mathscr{M}$ Beiträge fiir die in Rückdeckung übernommenen Versicherungen eingenommen, wovon $991047 \mathscr{M}$ für Rückversicherung weitergegeben wurden.

An dem gesamten Geschäfte der Gesellschaften in der Unfallversicherung war das deutsche Geschäft mit 12,6 v. H. der Beitragseinnahme und mit 26,1 v. H. der Ausgabe für Rückversicherungsbeiträge beteiligt.

In der Haftpflichtversicherung haben sechs ausländische Gesellschaften aus dem deutschen Geschä̈lt $7235431 \mathscr{N}$ eingenommen, davon $112525 \mathscr{M}$ oder $1,6 \mathrm{v}$. H. für die in Rückdeckung übernommenen Versicherungen; diese Beitragseinnahme betrug 30,5 v. II. des gesamten Haftpflichtrersicherungsgeschäfts. Es wurden im deutschen Geschäfte $1827128 \mathscr{H}$ oder 25,3 v. H. der Beitrïge, im Gesamtgeschüfte 7,7 v. H. der Beiträge für Rückversicherungen wieder ausgegeben.

Für selbst abgeschllossene Versicherungen hatten die Gesellschaften folgende Beträge bereitzustellen: $2390869 \mathscr{M}$ für Unfallversicherungställe, $2806307 \mathscr{M}$ für Haftpiliclit- 
versichcrungsfälle, $305847 \mathscr{N}$ für laufende Renten und $165475 \mathscr{N}$ für Beitragsrückgew:ihıbeträge, zusammen $5668498 \mathscr{M}$, für Versicherungshïlle aus den in Rückdeckung ülernommenen Versicherungen $5533 \mathscr{A}$ für Unfallversicherungsfille, $178881 \mathscr{A}$ für IIaftphlichtrersicherungsfille, $2605 \mathscr{A}$ für vorzeitig aufgelöste Versicherungen und $4035 \mathscr{H}$ für sonstige Lcistungen, zusammen $191054 \mathscr{M}$. Im Geschäftsjalır i 9 I 6 haben diese Gesellschaften insgesamt $5859552 \mathscr{N}$ für Schadenfälle, für Renten und für Prämienreserveergänzung verausgabt; in wclehem Umfange hieran die Rückversicherer beteiligt sind, kann nicht angrgeben werden. Diesen Schäılen stelıt eine Rohbeitragseinnahme - allerdings olıne Berücksichtigung der Úberträge - von $13280247 \mathscr{M}$ gegenübcr; von $1000 \mathscr{N}$ Beiträgen sind danach durchschnittlich $441 \mathscr{A}$ für Schäden ausgegeloen.

Im Vergleiche des Berichtsjahrs mit dem Vorjahr ist in der Unfillversicherung die Zahl der Versicherungsscheine von 134606 auf 133306 oder um 1,0 v. H. gefallen, die Beitragseinnalıme von $5906310 \mathscr{N}$ auf $6044816 \mathscr{A}$ oder um 2,3 v. H. gestiegen; bei cler II aftpflichtversicherung ist die Zahl der Versicherungsscheine von 396455 auf 388768 oder um 1,9 v. H. gefallen, die Beitragseinnalime von $7057628 \mathscr{M}$ auf $7235431 \mathscr{A}$ oder um 2,5 v. H. gestiegen.

Zur Beurteilung des Vermögensstandes der ausländischen Gesellschaften sind in diese Statistik die Bılanzen der Gescllschaften aufgenommen, wie sie für das ganze Geschäft (nicht nur für das deutsche Geschäft) gelten. Iabei ist möglichst nach denselben Grundsätzen und in denselben Formen vorgegangen wie bei den deutschen Unternehmungen; es bedarf daher hier keiner besoncleren Erllärungen.

Die Bilanz ist hier nur für sicben Gesellschaften zu geben (Tafel II 14), da die der Basler Lebens-Versicherungs-Gesellschaft nach Gruppe I genommen ist.

Ilinsichtlich der Kapitalanlagen dieser sieben Gesellschalten ist zu bemeṛken, daß die vier österreichischen Unternelımungen von ihrem ganzen Bestande von $120510660 \mathscr{M}$ in Grundbesitz $5349868 \mathscr{A}$ oder 4,4 v. H., in Ilypothelien $14175245 \mathscr{A}$ oler 11,8 v. II., in Darlchen an öfentliche Körperschaften $849709 \mathscr{H}$ oder $0,7 \mathrm{v}$ H., in Wertpapieren $93503797 \mathscr{A}$ oder 77,6 v. H., in Jarlehen auf' Wertpapiere $821275 \mathscr{H}$ oder $0,7 \mathrm{v} . \mathrm{H}$, in Darlehen auf Versicherungen $5104049 \mathscr{M}$ oder 4,3 v. II. und in Wechseln $646717 \mathscr{M}$ oder 0,5 v. II. angelegt lhatten, und daß ron dem gesamten Bestande (146977561 $\mathscr{M})$ der drei schweizerischen Gesellschaften $12168000 \mathscr{H}$ oder 8,3 v. H. in Grundbesitz, $54131286 \mathscr{N}$ oder 36,8 v. II. in Ilypotheken, $811511681 \mathscr{M}$ oder 54,8 v. H. in Wertpapieren und $166594 \mathscr{A l}$ oder 0,1 v. H. in Darlehen auf Versicherungen angelegt waren.

Fin eingehender Vergleich des ausländischen Geschäfts der deutschen Unternchmungen mit dem deutschen Geschälte der ausländischen Unternehmungen auf dem Gebiete der Unfall- und Iraftpflichtversicherung kann zwar nicht gegeben werden; indessen verdienen doch folgende Zahlen angefüilht zu werden:

Im Jahre 1916 betrugen die Rolıbeitragseinnalımen:

\begin{tabular}{|c|c|c|c|}
\hline & insgesamt & $\begin{array}{c}\text { für selbst } \\
\text { abgeschlossene Versicherungen }\end{array}$ & $\begin{array}{l}\text { für in Rīckdeckung } \\
\text { übernommene Versicherungen }\end{array}$ \\
\hline er deutschen Gesellschaften aus den & $1000 \mathscr{H}$ & $1000 \mu$ & $1000 \mathscr{K}$ \\
\hline $\begin{array}{l}\text { ausländıchen Geschisfie ......... } \\
\text { der ausländischen Gesellschaften aus } \\
\text { dem deutschen Geschälte........ }\end{array}$ & $\begin{array}{r}7573 \\
13280\end{array}$ & 6868 & $\begin{array}{l}705 \\
114\end{array}$ \\
\hline
\end{tabular}


Während also die Einnahmen an Rückversicherungsbeiträgen bel den deutschen Gesellschaften größer gewesen sind als bei den ausländischen Unternelımungen, bleiben die Beitragseinnahmen für das unmittelbare Geschält bei den deutschen Gesellschaften hinter den entsprechenden Einnalımen der auslïndischen Anstalten beträchtlich zurück; insgesamt haben die deutschen Gesellschaften für Unfall- und Iaftpflichtversicherungen rund $5707000 \mathscr{M}$ weniger an Beiträgen aus dem Auslande bezogen, als die ausländischen Gesellschaften aus dem Deutschen Reiche eingenommen haben.

Für Versicherungsleistungen sind im Jahre I916 gezahlt worden:

\begin{tabular}{|c|c|c|c|}
\hline & insgesamt & $\begin{array}{c}\text { fur sèlbst } \\
\text { abgeschlossene Versicherungen }\end{array}$ & $\begin{array}{l}\text { fur in Rückdeckung } \\
\text { ubernommene Versicherungen }\end{array}$ \\
\hline on & $1000 \mathscr{N}$ & $1000 \mathscr{H}$ & $1000 \mathscr{A}$ \\
\hline $\begin{array}{l}\text { ausländısihe Versichet ungen . } \\
\text { on den ausländ wchen Gesellschaften }\end{array}$ & $35^{87}$ & 2238 & I 349 \\
\hline für deutsche Versicherungen .. & 5859 & 5668 & 191 \\
\hline
\end{tabular}

Näheres hierüber findet man in der "Entwickelung " S. 13

\section{Gruppe III, Landwirtschaftliche Versicherung}

Für diese Gruppe kommen zur Zeit nur die Viehversicherung und die Hagelversicherung in Frage; sonstige landwirtschaftliche Versicherungen unterstanden in Jahre I 9 I 6 der Reichsaufsicht nicht.

\section{Viehversicherung}

Die Viehversicherung wird im Deutschen Reiche außer von einigen größeren Unternelimungen von mehreren tausend kleinen Vereinen von lokalcr Bedeutung betrieben. Davon unterliegt der Reichsaufsicht nur ein kleiner Bruchteil; dieser Bruchteil enthält wieder eine Anzahl Vereine ron so untergeordneter Bedeutung, daß sich ihre eingehendere statistische Betrachtung nur in Verbindung mit der Gesamtheit aller kleinen Unternelımungen lohnen würde, vgl. Veröffentlichungen des Kaiserlichen Aufsichtsamts 1918, S. 24 und 25.

Die vorliegende Statistik beschränkt sich daher auf 1 Aktiengesellschaft, die 26 wichtigsten größeren Gegenseitigkeitsvereine sowie auf 6 sonstige Unternelımungen. Die in den früheren Statistiken unter den sonstigen Unternehmungen aufgefiihrte Schlaclitviehversicherung von G. H. Koch \& Co. zu Berulurg ist fortgelassen worden, da der Betrieb am I. April i 9 I 6 vorläufig eingestellt wurde.

Die Aktiengesellschaft betätigt sich außer in der Viehlebens- und der Schlachtviehversicherung noch in melıreren sonstigen Viehversicherungsarten. Die Schlachtviehversicherung aufGegenseitigkeit des Vereins der Viehhändler zu Ilannover beschränkt sich auf die Schlachtviehversicherung. Die übrigen Gegenseitigkeitsvereine betreiben sämtlich die Viehlebensversicherung, 8 von ihnen gleichzeitig die Schlachtviehversicherung und 19 von ilhnen andere Arten der Viehversicherung. Danelen befaßt sich die Altenburger Vieh-Versicherungs-Gesellschaft auch mit der Sachschäden-IIaftpflichtversicherung und der CentralViehversicherungs-Verein in Berlin mit der Verkelursversicherung. Die sonstigen Unternehmungen (Fincelunternelımer) betätigen sich sämtlich und in erster Linie in der Schlıchtviehversicherung; außerdem betreibt die Ostrauer Schlachtvieh-Versicherung auch die 
Viehtebensversieherung und die Schlesische Schlachtvieh-Versicherung auch die kurzfristige Nutzvieh- und Kuhkalbenversicherung.

Auslindische Unternelımungen waren in der Viehversicherung zum Geschäftsbetrieb im Deutschen Reiche nicht befugt.

Nach Tafel III 1 hatten die sämtlichen in Betracht genommenen 33 Unternehmungen im Berichtsjahre Versicherungen im Betrage von 1081,601 Millionen Mark in Kraft; davon kommen 47 $\overline{7}, 758$ Millionen auf die Aktiengesellschaft, j()2,;69 Millionen (darunter 291,386 Millionen Mark gegen feste Beiträge) auf dıe 26 Gegenseitigkeitsvereine und 101,134 Millionen auf die 6 andern Unternehmer. Rückversiçherungen finden sich nur bei der Aktiengesellschaft und bei den Gegenseitigkeitsvereinen. Von der Aktiengesellschaft waren 5,6̈2 Millionen Mark in Rückdeckung übernommen und $\mathbf{6 1 , 1 4 6}$ Millionen Mark (gegen 44,393 Millionen Mark im Vorjahre) in Rückdeckung gegeben, von dem Gesamtbetrage der Versicherungen der Gegenscitigkeitsvereine waren 6,013 Millionen Mark in Rückdeckung übernommen und 52,149 Millionen Mark in Rückdeckung gegeben, so daf für eigene Gefahr die Aktiengesellschaft 416,612 Millionen Mark und die Gegenseitigkeitsvereine 450,620 Millionen Mark versichert hatten.

Der Gang der Versicherungssummen in den letzten Jahren ist in der beigegebenen Tafel billlich dargestellt.

Die Reinbeitragseinnahme aller Unternehmungen, d.h. die im Laufe des Jahres von den Versicherten eingenommenen Beitrïge nach Abıug der Rückversicherungsbeiträge, lat

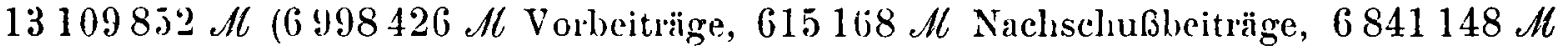
feste $B$ (نiträge und $1344890 \mathscr{N}$ Rückversicherungsbeitr:ige, Tafel Ill j) betragen.

Gegen feste Beiträge werden von den Gegenseitigkeitsvercinen in der Regel abgeschlossen: die Schlachtvichversicherung und die in Tafel III 2 unter sonstiger Vichversicherıng zusammengefaßten Arten, nämlich die Rückversicherung, die Vichtransport-, die Manöver-, Weide-, Stuten-, Operations-, Ausstellungsversicherung usw.

Als Schäden für eigene Rechnung ohne Schadencrmittelıngskosten, aber unter Berücksichtigung des Zuwachses oder der Abnahme der Schadenrücklage und unter Einschluß des Erlöses für verwertetes Vieh sind $10262209 \mathscr{A l}$ zu verreclinen gewesen.

Fine Statistik über die Ursachen der Entschädigungspllicht im Jahre 19 1 6 ist in dem Geschüftsberichte des Aufsichtsamts für I916 gegeben, vgl. Veróflentlichungen I917 S. $103 \mathrm{ff}$.

Die Übersicht S. 50* und $51^{*}$ zeigt, wie sich die Beiträge und die Schäden auf die verschiedenen Versicherungsweisen, die Vieharten und deren Stückzahl und Wert verteilen. Sie beruht auf denselben Grundlagen wie 'Tafel III 2 ; es sind daher auch in dieser Ubersicht die Anteile der Rüchversicherer üherall eingeschlossen. Die Beiträge, von denen die Rabatte niclit abgesetzt sind, sind die Einnahmen im Geschäftsjalır; die Schäden sind die im Laufe des Geschiiftsjalırs entstandenen Schüden. In dieser Zusammenstellung kommt beispielsweise besonders deutlich zum Ausdruck, wie sehr verschieden die Verhältnisse bei den verschiedenen Versicherungsarten liegen.

Seit dem Jahre I 905 hat der gesamte Versicherungsbestand nach Vieharten verteilt werden können; es mag deshalb hier eine kleine Ubersicht über die Stückzahl der versicherten Tiere und der, zugehörigen Versicherungssummen für die letzten 5 Jalıre Platz finden, vgl S. 52*. In betreff der vorhergehenden Jahre sei auf die früheren Jahrgänge der Statistik und auf die "Entwickelung“ S. 29 verwiesen. 


\begin{tabular}{|c|c|c|c|c|c|c|}
\hline \multirow[t]{2}{*}{$V, e h$ a $r t$} & \multirow[t]{2}{*}{ Stiuckzahl } & \multirow{2}{*}{$\begin{array}{c}\text { Ver- } \\
\text { sicherungs- } \\
\text { summen } \\
\mathscr{A}\end{array}$} & Beitrãge & \multirow[t]{2}{*}{$\begin{array}{l}\text { Zahl der } \\
\text { Schäden }\end{array}$} & \multirow{2}{*}{$\begin{array}{c}\text { Betrag der } \\
\text { Schäden } \\
\text { einschl. } \\
\text { Erlös } \\
\\
\text { A }\end{array}$} & \multirow{2}{*}{$\begin{array}{l}\text { Erlös } \\
\mathscr{A}\end{array}$} \\
\hline & & & $\mathscr{M}$ & & & \\
\hline 1 & 2 & 3 & 4 & 5 & 6 & 7 \\
\hline & & & & & \multicolumn{2}{|c|}{ Viehversicherung } \\
\hline Pferde (Maultiere, Esel) ..... & $2463^{82}$ & 316609374 & 10310313 & 11360 & 7234422 & 723090 \\
\hline Rund ieh $\ldots \ldots \ldots \ldots \ldots$ & 1057368 & 539940197 & 3074461 & 27465 & 3069612 & I 343537 \\
\hline Sthafe und Ziegen $\ldots \ldots \ldots$ & 185433 & 18689546 & 69300 & 1139 & 40069 & 4650 \\
\hline Schweme $\ldots \ldots \ldots$. $\ldots \ldots$ & 877574 & $20637275^{\circ}$ & 1079829 & I 5394 & 733672 & 246250 \\
\hline Soustiges Klemvieh ..... . & 249 & 49370 & 2710 & 8 & I 676 & $3^{8}$ \\
\hline Zusammen .... & 23676106 & 1081661237 & 14536613 & 55366 & 11079451 & 2317565 \\
\hline
\end{tabular}

Viehlebensversicherung (1 Aktiengesellschaft,

\begin{tabular}{|c|c|c|c|c|c|c|}
\hline Pferde (Maultiere, Esel) .. .. & 149312 & 185549745 & 7510204 & 6395 & 5614203 & 663802 \\
\hline Rindrich : $\quad \ldots \ldots$ & 43578 & 21807233 & 538499 & 809 & 334346 & 111535 \\
\hline Schafe und Ziegen $\quad . .$. . & 10313 & 673265 & 27783 & 676 & 28309 & 2375 \\
\hline Sthweme... . . $\quad \ldots \quad \ldots \quad$. & 47114 & 8646617 & 377213 & 2804 & 216873 & 28208 \\
\hline Sonstıges Klemı ıch..... & 226 & 48795 & 2698 & 8 & I 676 & 38 \\
\hline Zusammen .... & 250543 & 216725655 & 8056397 & 10692 & 6195407 & 805958 \\
\hline
\end{tabular}

\section{Schlachtviehversicherung}

\begin{tabular}{|c|c|c|c|c|c|c|}
\hline Pferde (Maultiere, Esel) ..... & 2 & 200 & 10 & 一 & - & - \\
\hline lindrieh $\ldots \ldots \ldots \ldots \ldots \ldots$ & 798340 & 391767493 & I 94957 I & 25912 & 2481220 & 1154855 \\
\hline Schate und Ziegen $\ldots \ldots \ldots$ & 124377 & 13190661 & 34323 & 377 & 6744 & 1890 \\
\hline Schweme............. & 675491 & 170745700 & 578826 & 11441 & 434659 & 214163 \\
\hline Sonstyges Klem ieh. . ... . . & 23 & 575 & 12 & 一 & - & - \\
\hline Zusammen ... & 1598233 & 5750704629 & $256274 ?$ & 37730 & 2922623 & 1370908 \\
\hline
\end{tabular}

\section{Sonstige Viehversicherung}

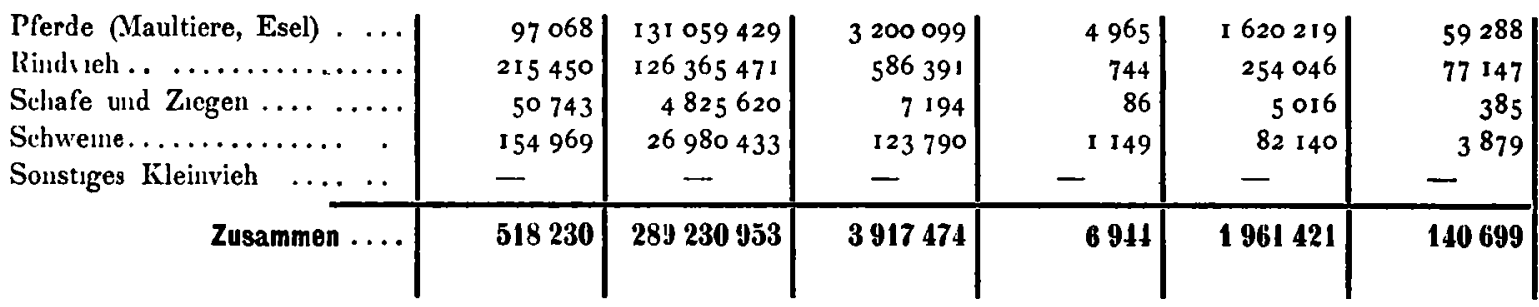

Anmerkung: Ein - bedeutet, daß die beobachteten Fălle weniger als $1 / 2 \%$ ausmachen. 
Viehversicherung

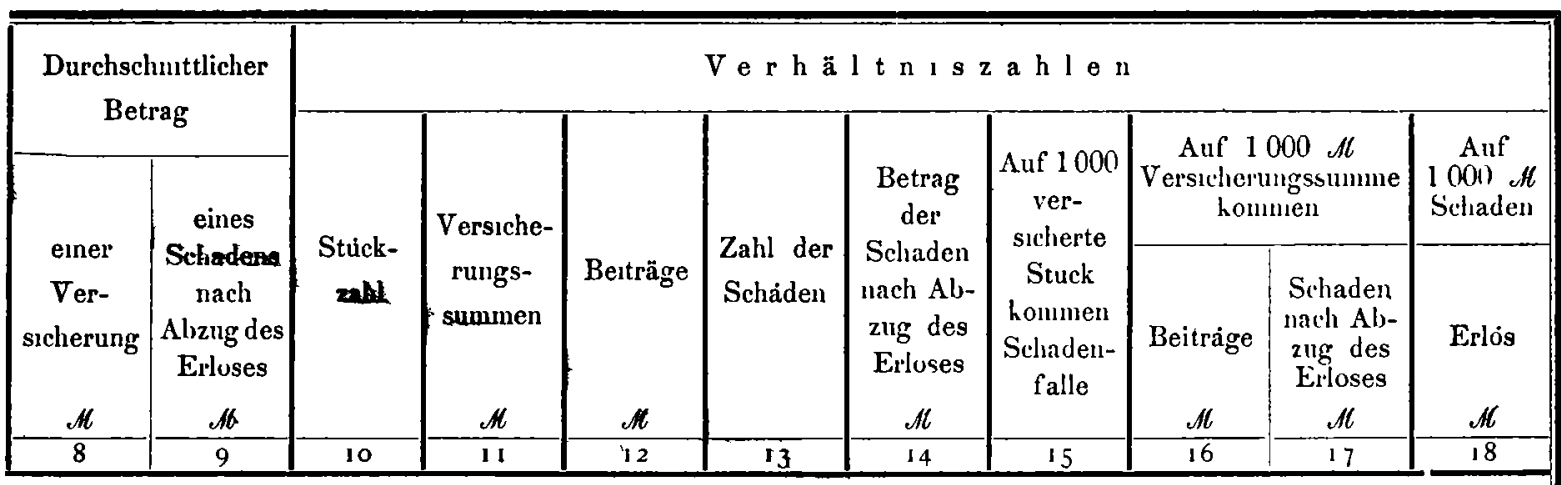

insgesamt

\begin{tabular}{|c|c|c|c|c|c|c|c|c|c|c|}
\hline I 285 & 573 & 104 & 293 & 709 & 205 & $743 \mid$ & 461 & 33 & 2 I & 100 \\
\hline 5 I I & 63 & 447 & 499 & 212 & 496 & 197 & 26 & 6 & 3 & 438 \\
\hline Ior & 31 & 78 & I 7 & 5 & 20 & 4 & 6 & 5 & 2 & 116 \\
\hline 235 & 32 & 371 & 191 & 74 & 279 & $5^{6}$ & 18 & 5 & 2 & 336 \\
\hline I 98 & 205 & . & . & . & & - & 32 & 55 & 33 & 23 \\
\hline 457 & 158 & 1000 & 1000 & 1000 & 1000 & 10100 & 23 & 13 & 8 & 209 \\
\hline
\end{tabular}

25 Gegenseitigkeitsvereine und 1 sonstige Unternehmung)

\begin{tabular}{|c|c|c|c|c|c|c|c|c|c|c|}
\hline J 243 & 774 & 596 & 8561 & $88_{3}$ & 5981 & 919 & 43 & $3^{8}$ & 27 & I I 8 \\
\hline 500 & 275 & 174 & IOI & 67 & 76 & 41 & 19 & 25 & 10 & 334 \\
\hline 65 & $3^{8}$ & 41 & 3 & 3 & 63 & 5 & 66 & $4 I$ & 39 & 84 \\
\hline I 84 & 67 & I 88 & 40 & 47 & 262 & 35 & 60 & 44 & 22 & I 30 \\
\hline 216 & 205 & I & & & $\mathbf{I}$ & & 35 & 55 & 34 & 23 \\
\hline 865 & 504 & 1000 & 1000 & 1001 & 1000 & 1000 & 43 & 37 & 25 & $130^{\circ}$ \\
\hline
\end{tabular}

(16 Unternehmungen)

\begin{tabular}{|r|r|r|r|r|r|r|r|r|r|r|}
100 & - & $\cdot$ & 6 & & - & - & - & 50 & - & - \\
491 & 51 & 499 & 680 & 761 & 687 & 855 & 32 & 5 & 3 & 465 \\
106 & 13 & 78 & 23 & 13 & 10 & 3 & 3 & 3 &. & 280 \\
253 & 19 & 423 & 297 & 226 & 303 & 142 & 17 & 3 & - & 493 \\
25 & - &. & &. & - & - & - & 21 & - & - \\
\hline 360 & 41 & 1040 & 1000 & 1000 & 1000 & 1000 & 24 & 4 & 3 & 469
\end{tabular}

(21 Unternehmungen)

\begin{tabular}{|r|r|r|r|r|r|r|r|r|r|r|}
1350 & 314 & 187 & 453 & 818 & 715 & 857 & 51 & 24 & 12 & 37 \\
587 & 238 & 416 & 437 & 149 & 107 & 97 & 3 & 5 & 1 & 304 \\
95 & 54 & 98 & 17 & 2 & 12 & 3 & 2 & 1 & 77 \\
174 & 68 & 299 & 93 & 31 & 166 & 43 & 7 & 5 & 3 & 47 \\
- & - & - & - & - & - & - & - & - & - & - \\
\hline 558 & 262 & 1000 & 1000 & 1000 & 1000 & 1000 & 13 & 14 & 6 & 72
\end{tabular}




\begin{tabular}{|c|c|c|c|c|c|c|c|c|}
\hline \multirow{2}{*}{ Geschäftsjahr } & \multicolumn{3}{|c|}{ Viehlebeısı ersicherung } & \multicolumn{2}{|c|}{ Sehlachtr iehs ersicherung } & \multicolumn{3}{|c|}{ Sonstige Viehnetsicherung } \\
\hline & Pferde & Rundweh & Schweine & Rundvieh & Schweine & Pferde & Rindvieh & Schweiue \\
\hline & \multicolumn{8}{|c|}{ Zahl der versicherten Tiere } \\
\hline 1912. & 225035 & 81446 & 32 I 8 & 762246 & 2105806 & 56834 & 118642 & 343890 \\
\hline $1913 \ldots \ldots$ & 223382 & 66601 & 27156 & 706607 & 1945518 & $6494 \mathrm{I}$ & $1055^{89}$ & 265077 \\
\hline 1914. & $22069 I$ & 54077 & 25860 & 700040 & 1895 I 54 & 69775 & 97898 & 236606 \\
\hline 1915 . & 179394 & 46496 & 22455 & 778650 & 1255441 & 72816 & 93067 & $16656 r$ \\
\hline \multirow[t]{2}{*}{1916} & 149312 & 43578 & 47114 & 798340 & 675491 & 97068 & $21545^{\circ}$ & 154969 \\
\hline & \multicolumn{8}{|c|}{ Versicherungssummen in $1000 \mathscr{A}$} \\
\hline 1912. & 191791 & 30888 & 2878 & 229339 & 248836 & $3952 \mathrm{I}$ & 48121 & 30836 \\
\hline 1913 & 193609 & 25818 & 2654 & 224544 & 232793 & 50698 & 48725 & 24883 \\
\hline $1914 \ldots \ldots$ & 196692 & 22564 & 2630 & 232426 & $23^{6} 5^{6} 3$ & 52621 & 44060 & 22677 \\
\hline $1915 \ldots \ldots$ & $17245^{2}$ & 19277 & 2424 & 307539 & 213817 & 70288 & 40679 & 19657 \\
\hline $1916 \ldots \ldots$. & $18555^{\circ}$ & 21807 & 8647 & 391767 & 170746 & 131059 & 126366 & 26980 \\
\hline
\end{tabular}

\section{Die in Tafel III 3 zum Vergleiche des Bcrichtsjahrs mit dem Vorjahr aufgeführten} Zahlen sind bei den Versicherungssummen nicht streng vergleichbar, weil an einigen Stellen nicht der Betrag der überhaupt in Kraft gewesenen Versicherungen, sondern der Betrag der am Ende des Jahres laufenden Versicherungen eingestellt wcrden mußte. Bei der Aktiengesellschaft haben die Versicherungssummen um 171,448 Millionen Mark oder 56,0 v. H. zugenommen, die Beiträge um 0,052 Millionen oder 2, I v. II. und die Schadenbeträge (ohne Abzug des Erlöses) um 0,352 Millionen oder 12,9 v. II. abgenommen. Bei den Gegenseitigkeitsvereinen haben die Versicherungssummen um 134,184 Millionen Mrark oder 36,4 v. H., die Beiträge um 2,046 Dillionen oder 25,2 v. H. und die Schadenbeträge (olıne Abzug des Erlöses) um 0,325 Millionen oder 4,6 v. H. zugenommen. Der Vergleich von 1915 mit 1914 hatte nach der vorjührigen Statistik hinsichtlich der Aktiengesellschaft bei den Versicherungssummen eine Zunahme um 1,619 Millionen Mrark oder 0,5 v. H., bei den Beitrïgen eine Zunalime um 0,076 Millionen oder 3,2 v. II. und bei den Schäden eine Abnalme um 0,296 Millionen oder 9,8 v. H. und hinsichtlich der Gegenseitigheitsvereine bei den Versicherungssummen eine Alnahme um 10,417 Millionen Mark oder 2,7 v. H., bei den Beiträgen eine Abnalıme um 0,9-6 Nillionen oder 10,3 v. H. und bei den Sclı̈̈len eine Alnalıme um 1,174 Millıonen oder 14,3 v. H. ergelsen.

Bei den sonstigen Unternehmungen haben die Versicherungssummen um 69,768 Millionen oder 40,8 v. H., die Beitriage um 0,436 Nillionen oder 43,7 v. $\mathrm{H}$. und die Schadenzalilungen um 0,522 Millionen oder 51,2 v. H. algenommen.

Der Einfluß des Krieges hat sich bei den sonstigen Unternehmungen im Jahre i 9 I 6 noch weit stärker geltend gemacht als I 915 und I 9 I 4, während bei den Gegenseitigkeitsvereinen eine erhebliche Besserung zu verzeichnen ist.

Die Gewinn- und Verlustrechnung (Tafel III 4) stellt nur das Ergebnis des Berichtsjahrs dar; der Gewinnvortrag aus dem Vorjahr ist fortgelassen, auch sind die Prämienreserven und Schadenrücklagen nicht mit ilırem ganzen Betrag am Anfange des Jahres in Einnahme und am Schlusse des Jahres in Ausgabe nachgewiesen, sondern es sind nur die Änderungen, welche der Betrieb des Jahres zur Folge hatte, verrechnet (vgl. Tafel III 5).

Die gesamte Beitragseinnalime hat im Jahre 1916 $14454742 \mathscr{M}$ betragen. Wie sich dieser Betrag auf Vorbeiträge, Nachschußbeiträge und feste Beiträge verteilt, ist 
bereits oben angegeben; es braucht hier nur noch hinzugefügt zu werden, daß die Nachschü̈sse 8,8 v. H. der Vorbeiträge aller Unternelımungen und 32,0 v. H. der Vorbeiträge derjenigen Unternehmungen ausgemacht haben, welche im Berichtsjahr überhaupt Nachschüsse erhoben haben. Verrechnet man auf die Beiträge die Prämienübertrïge und die Rückversicherungsbeiträge, so erhält man die in die Betricbsrechnung eingestellte Beitragseinnahme. Die von einigen Vereinen durch die Gewinn- und Verlustrechnung gefülırten "Nachschußversicherungsbeiträge " oder "Nachschußrückversicherungsbeiträge " sind hier fortgelassen; es handelt sich nämlich um Beiträge für eine Nebenversicherung gegen etwaigen Nachschuß, die der Verein von seinen Mitglichern einzieht, um sie zumeist vollständig an andere Versicherungsgesellschaften ab/uführen, also um durchlaufende Posten, die den Geschäftsbetrieb nicht weiter bcrühren. Die Leistungen der Versicherer aus diesen Versicherungen sind bei den Nachschüssen eingestellt.

Die Nebenleistungen der Versicherten haben bei den Gegenseitigkeitsvereinen im Jahre 19 $6514228 \mathscr{M}$, das sind 4,6 v. H. der ganzen Beitragseinnahme oder 7,3 v. H. der Vorbeiträge, bei der Aktiengesellschaft $13471 \mathscr{A}$, das sind 0,5 v. H. der ganzen Beitragseinnalıme, betragen. Dabci ist zu bemerken, daß bei den meisten Versicherungsgesellschaften als satzungsmäßige Leistung der Mitglieder zur gesetzlichen Rücklage bestimmte IUndertsïtze des Vorbeitrags verrechnet und als Teile des Vorbcitrags nachgewiesen werden, also nicht bei den Nebenleistungen erscheinen.

Der Erlös aus verwertetem Vieh belief sich auf $2307206 \mathscr{M}$, d. h. 22,5 $\mathscr{M}$ von je $100 \mathscr{H}$ Schäden fül eigene Rechnung. IIichloei ist zu beachten, daß zum 'Teil nicht nur der wirklich erzielte Erlós oder der bedingungsgemäß gestattete Mindesterlös in Rechnung gestellt ist, sondern auch vielfach, insbesondere bei Versicherungen gegen Minderwert, der Unterschied zwischen Versicherungssumme und gezahltem Schadenbetrage angerechnet ist.

An Schadenermittelungskosten wurden $235821 \mathscr{N}$ oder $2,3 \mathscr{N}$ auf je $100 \mathscr{H}$ Schäden einschließlich Erlös gezahılt; an eigenen Verwaltungskosten und Steuern wurden $3099244 \mathscr{M}$ oder 25,2 v. H. der Beiträge für eigene Rechnung (Tafel III 5 Sp. 8) verausgabt.

Aus der Bilanz für Ende I916, die nur fïr die Aktiengesellschaft und die Gegen- Die Bilanz seitigkeitsvereine gegeben wird, mögen folgende Posten angefülrt werden:

\begin{tabular}{|c|c|c|c|}
\hline Vermögenswerte & 1 Ahtiengesellschaft & 27 Gegenscitıgkei & tsvercine \\
\hline Verpflichtungen der Ahtionäre oder Garanten... & $750000 \mathscr{H}$ & - & $A$ \\
\hline Kapitalaulagen..$\ldots \ldots \ldots \ldots \ldots \ldots \ldots \ldots$ & 628965. & 6444094 & • \\
\hline Forderungen..$\ldots \ldots \ldots \ldots \ldots \ldots \ldots$ & 555051. & 2994739 & * \\
\hline Kasseubestand $\ldots \ldots \ldots \ldots \ldots \ldots \ldots$ & 10652 & 203013 & . \\
\hline Verbindlichkeiten & & & \\
\hline Aktien- oder Garantıchapital $\ldots \ldots \ldots \ldots \ldots$ & $1000000 \mathscr{H}$ & - & $\mathscr{M}$ \\
\hline Gesetzliche Rüchlago .............. & 100000 & 4786361 & * \\
\hline Sonderrü hlagen $\ldots \ldots \ldots \ldots \ldots \ldots \ldots \ldots$ & 295407 . & 310301 & - \\
\hline Prămienübcrträge... . . . . . . . . . . . . & 153842. & 2232486 & " \\
\hline Schadenrücklage $\ldots \ldots \ldots \ldots \ldots \ldots$ & $5^{8094} \cdot$ & $55^{8864}$ & " \\
\hline
\end{tabular}

Die Kapitalanlagen der $A$ ktiengesellschaft und der Gegenseitigkeitsvereine zusammen bestehen zu 81,8 v. H. in Wertpapieren, zu 4,7 v. H. in Grundbesitz und zu 13,5 v. H. in Hypotheken. Im Vergleiche mit dem Bestand am Ende des Vorjahrs waren im 
Besitze dieser Unternehmungen Ende I 9 I 6 vorhanden an Wertpapieren $1061268 \mathscr{M}$ (dem Nennwert nach $1189700 \mathscr{M}$ ) und an Grundbesitz $73686 \mathscr{M}$ mehr, dagegen an Hypotheken $8523 \mathscr{M}$ weniger. Die Forderungen bestehen bei der Gesamtheit zu 45,1 v. H. in Rückständen der Versicherten (einschl. der noch nicht gezalılten Nachschüsse); ins Gewicht fallen bei einigen Gesellschaften auch die Ausstände bei Agenten.

Die gesetzlichen Rücklagen haben gegen die Bilanz für Ende I 9 I 5 um $653094 \mathscr{M}$ oder um 15,4 v. H. zugenommen; die Sonderrücklagen vermehrten sich um $357424 \mathscr{M}$ oder 144,0 v. $\mathrm{H}$.

Das ansIandigs he Geschaft

. folgende Nachrichten dienen.

Im Jahre I916 haben wie in den Vorjahren nur 5 Versicherungsvereine auch im Auslande Geschäfte abgeschlossen, davon je einer in Belgien, Luxemburg und in der Schweiz und je zwei in Dänemark und Österreich. Diese Vereine hatten am Ende des Jalıres im Ausland eine Summe von $3577115 \mathscr{A}$ versichert. An Beiträgen haben sie im Berichtsjalır $210944 \mathscr{A}$ eingenommen, an Schäden sind $165217 \mathscr{N}$ gezalılt. Das ausländische Geschält war also in der Viehversicherung nur unbedeutend; es betrug $3,3^{\circ}$ vom Tausend der gesamten Versicherungssumme, 16,1 vom Tausend der Beitragseinnalıme und 16,1 vom Tausend der Schäden der Unternelımungen.

\section{Hagelversicherung}

Von den im Deutschen Reiche im Jahre 1916 vorhanden gewesenen Hagelversicherungsunternelımungen unterstanden dem Aufsichtsamte 4 Aktiengesellschaften und 14 Gegenseitigkeitsvereine. Davon sind in diese Statistik außer den Aktiengesellschafien 12 Vereine aufgenommen; die übrigen zwei sind von ganz lokalem Charakter und geringer Bedeutung, so daß von ilınen nicht wohl alle Angaben, welche diese Statistik bringt, gefordert werden können.

Drei von den hier behandelten 12 Gegenseitigkeitsvereinen betreiben auch die Feuerversicherung, nämlich die Greifswalder, die Mecklenburgische und die Schwedter Hagel- und Feuer-Versicherungs-Gesellschaft; eine, die Greifswalder, betreibt auch die Baulast- und die Einbruchdicbstahlversicherung. Alle drei halten aber die Versicherungszweige in ilıren Büchern getrennt und geben eine vollständige Gewinn- und Verlustrechnung und Bilanz für jeden Zweig. Diese vollständige Trennung ist auch hier beibehalten worlen, so daß sich die hier für diese Gesellschaften gegebenen Zahlen lediglich auf die Hagelversicherung beziehen, wie die für dieselben Gesellschaften bei Gruppe IV gegebenen Zahlen lediglich das Feuerversicherungsgeschäft betreffen.

Ausländische Hagelversicherungsgesellschaften haben, soweit bekannt, im Jahre 19 I 6 im Deutschen Reiche nicht gearbeitet. 
Der Umfang des Geschäfts im Berichtsjahre wirl durch folgende Zahlen bezeichnet. Es kamen insgesamt 3874,263 Millionen Mark auf 488840 selbst alogeschlossene Versicherungen oder im Durchschnitt $7925 \mathscr{M}$ auf einen Versicherungsschein.

Hierin sind 188,611 Millionen Mark enthalten, die zu 15812 Kollcktiv- oder Gemeindeversicherungen gehören, welche in der vorstehenden Zahl der Versicherungen je nur als eine Versicherung gezählt sind, aber 163965 Teilnehmer hatten. Die durchschnittlich von einem Versicherten versicherte Summe stellt sich demnach bei der Gesamtheit auf $6082 \mathscr{M}$ und bei den Gemeinde- und Kollektivversicherungen allein auf $1150 \mathscr{M}$.

Von den Versicherungen kommen auf die Aktiengesellschaften 947,521 Millionen Mark und 121366 Versicherungsscheine oder $7807 \mathscr{A}$ im Durchschnitt für den Schein und auf die Gegenseitigkeitsvereine 2926,742 Mlillionen Mark und 367474 Versicherungen oder $7964 \mathscr{M}$ im Durchschnitt für den Schein.

Die Rückversicherung hatte keine große Bedeutung. Es hatten in Rückdeckung übernommen vier Aktiengesellschaften zusammen 128,054 Millionen Mark und in Rückdeckung gegeben vier Gesellschaften 121,306 Millionen Mark, so daß die Aktiengesellschaften zusammen von 1075,575 Millionen Mark überhaupt übernommener Versicherungen 9j4,269 Millionen für eigene Rechnung behalten haben. Von den Gegenseitigkeitsvereinen haben drei 2,324 Millionen Mark in Rückrleckung übernommen und 3,540 Millionen Mark in Rückdeckung grgeben, so daß bei diesen Anstalten 2 925,526 Millionen Mark für eigene Reclinung blieben.

Die Reinbeitragscinmalımon, d. h. die im Laufe des Jahres von den Versicherten Die Beitrage eingenommenen Beitrïge, vermindert um die ausgegebenen Rückversicherungsbeiträge, haben insgesamt betragen $45564422 \mathscr{M}$, wovon $1126(3108 \mathscr{M}$ auf die Alitiengesellschaften und $34298314 \mathscr{A}$ auf die Gegenseitigkeitsvereine entfielen (Tafel III 11).

Als Schäden für eigene Rechnung, ohne die Frmittelungskosten, aber unter Berücksichtigung der Änderung der Schadenrücklage im Laufe des Jahres (vgl. Tafeln III 11 und 13), sind insgesamt $33252474 \mathscr{M}$ zu verrechnen gewesen: $4512066 \mathscr{A}$ bei den Aktiengesellschaften und $28740408 \mathscr{M}$ bei den Gegenseitigkeitsvereinen. Als jedenfalls nicht zu kleine Zahl der Schäden (weil bei drei Gesellschaften nicht die Zahl der Schäden, sondern die Zahl der Anmeldungen angegeben ist) darf für die Gesamtheit 54302 angenommen werden, nämlich 13711 für die Aktiengesellschaften und 40591 für die Gegenseitigkeitsvereine. Es kommen also auf den Schadenfall im Durchschnitt mindestens: bei der Gesamtheit $612 \mathscr{M}$, bei den Aktiengesellschaften $329 \mathscr{M}$ und bei den Gegenseitigkeitsvereinen $708 \mathscr{M}$.

Als Entsclıädigungsabzüge sind insgesamt $2609369 \mathscr{M}$ einbehalten worden, $225478 \mathscr{M}$ von den Aktiengesellschaften und $2383891 \mathscr{M}$ von den Gegenseitigkeitsvereinen. Die Abzüge bestanden bei den Aktiengesellschaften ausschließlich in Anteilen der Geschïdigten an den Ermittelungskosten; von den Abzügen der Gegenseitigkeitsvereine waren $899134 \mathscr{M}$ für Ermittelungskosten, $1483988 \mathscr{M}$ für die gesetzlichen Rücklagen und $10769 \mathscr{N}$ für andere Zwecke bestimmt.

Die Prämienüberträge und die Schadenrücklage spielen bei der Hagelversicherung naturgemäß nur eine ganz untergeordnete Rolle (vgl. Tafel III 13); man kann daher von diesen Rückstellungen absehen und folgende Zahlen als zutreffend annehmen: 
Von $1000 \mathscr{H}$ Beiträgen wurden für Schadenzahlungen nach Verrêchnung der Entschäligungsabzüge verwendet:

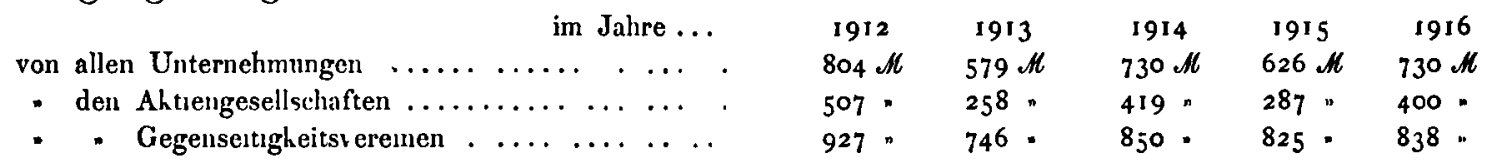

Auf $1000 \mathscr{H}$ Versicherungssumme kamen:

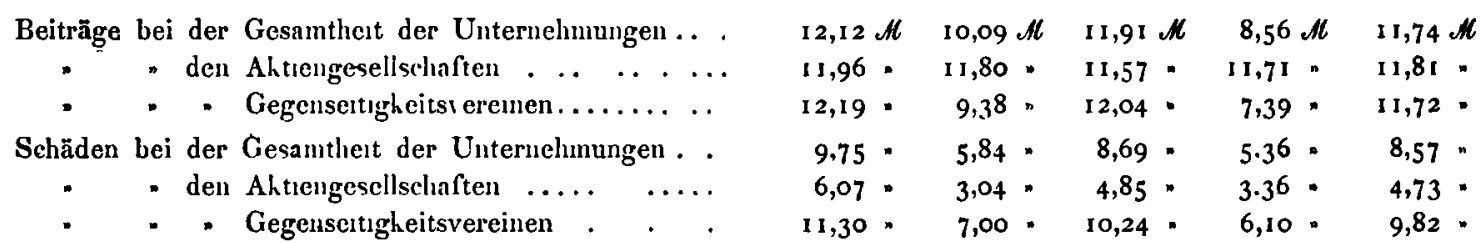

Vergleich des Berkhtsjahrs mit dem Yorjahre

Eine Vergleichung des Berichtsjahrs mit dem Vorjahre führt zu folgenden $\mathrm{Er}$ gebnissen ('Tafel III 11).

Die Zahl der Versicherungen hat bei der Gesamtheit der Unternehmungen um 13347 oder 2,3 v. IH. al)genommen; im besonderen hat sie bei den Aktiengesellschaften um 23480 oder 10,1 v. H. alogenommen und bei den Gegenseitigkeitsvereinen um 10133 oder 2,8 v. II. zugenommen. Die Versicherungssummen sind bei der Gesamtheit der Unternehmungen um $384,9+0$ Millionen Mark oder 10,6 v. H. gestiegen, und zwar bei den Altiengesellschaften um 9,228 Millionen Mark oder 0,9 v. H. und bei den Gegenseitigkeitsvereinen um 375,712 Millionen Mark oder 14,7 v. H. Die Reinbeitragseinnahme (einschl. Nachsschüsse) hat im Berichtsjahre gegenüber dem Vorjahre bei den Aktiengesellschaften um $187082 \mathscr{A}$ oder 1,7 v. II. und bei den Gegenseitigkeitsvereinen um $15436702 \mathscr{M}$ oder 81,8 v. H. zugenommen. Die Schälen für eigene Rechnung sind bei den Aktiengesellschaften um $1330291 \mathscr{N}$ oder 41,8 v H. und bei den Gegenseitigkeitsvereinen um $13174410 \mathscr{N}$ oder 84,6 v. H. höher gewesen als im Vorjahre.

Die in Tafel III 12 gegebene Gewinn- und Vcrlustrechnung stellt nur das Ergebnis des Berichtsjahrs dar; der Gewinnvortrag aus dem Vorjahre ist fortgelassen; auch sind die sonstigen Uberträge nicht mit ihrem vollen Betrage zu Anfang des Jahres in Einnahme und am Schlusse des Jahres in Ausgabe nachgewiesen, sondern es sind nur die Änderungen, welche der Betrieb des Geschäftsjahrs zur Folge hatte, verrechnet (vgl. Tafel III 13).

Die Beiträge haben bei den Aktiengesellschaften $12612331 \mathscr{M}$ betragen, wovon für Rückversicherung $1346223 \mathscr{M}$ wieder ausgegeben wurden. Die Gegenseitigkeitsvercine haben $21895400 \mathscr{N}$ Vorbeitrag und 12463776 $\mathscr{N}$ Nachschußbeitrag erhoben; für Rückversicherung haben sie $60862 \mathscr{N}$ wieder ausgegeben; der Nachschußbeitrag machte 56,9 v. H. von dem Vorbeitrag aus, oder wenn man die Greifswalder, die Schleswig-IIolsteiner in Kiel, die Neubrandenburger, den Holsteinischen II.V.V. in Neustadt und die Oldenburger H.V.G. ausschließt, weil sie die Beiträge durch Umlage nach Bedarf aufbringen, 70,8 v. II. Gewisse von mehreren Gegenseitigkeitsvereinen unter der Bezeichnung "Rückversicherungsbeiträge " durch die Gewinn- und Verlustrechnung gefülırte Posten sind hier fortgelassen; es handelt sich nämlich um Beiträge für eine Nebenversicherung gegen etwaigen Nachschuß, die der Verein von seinen Mitgliedern einzicht, um sie meist vollständig an andere Versicherungsgesellschaften abzuführen, also um durch- 
laufende Posten, die das Geschäftsergebnis nicht weıter beruhren. Die Leistungen der Versicherer aus diesen Versicherungen sind bei den Nachschüssen eingestellt.

An Nebenleistungen hatten die Versicherten zu zahlen bei den Aktiengesellschaften $88386 \mathscr{M}$, das sind 0,7 v. H. der Beiträge, und bei den Gegenseitigkeitsvereinen $2765077 \mathscr{M}$, das sind 8,0 v. H. der gesamten Beiträge oder 12,6 v. H. der Vorbeiträge. Die Nebenleistungen bestehen bei den Gegenseitigkeitsvereinen zum größten Teile $(22749 ; 5 \mathscr{M})$ in satzungsmäßigen Beiträgen zur gesetzlichen Rücklage, die entweder nach der Höhe des Vorbeitrags oder der Entschädigung bemessen werden; setzt man diese ab, so bleiben $490102 \mathscr{M}$, das sind 1,4 v. H. der gesamten Beitrïge oder 2,2 v. H. der Vorbeiträge. Die von einigen Gegenseitigkeitsvereinen erhobenen Legegelder (Sicherheitsleistungen) sind hier außer acht gelassen.

An Schadenermittelungskosten sind ohne die oben erwälnten Abzüge bei allen Unternelımungen $1248481 \mathscr{N}$ oder $3,8 \mathscr{N}$ auf je $100 \mathscr{N}$ Schadenzahlung, bei den Aktiengesellschalten $420885 \mathscr{M}$ oder $9.3 \mathscr{M}$ und bei den Gegenseitigkeitsvereinen $827596 \mathscr{M}$ oder 2,9 $\mathscr{M}$ auf je $100 \mathscr{M}$ Schäden entstanden.

Das Geschäft des Jahres I 9 I 6 hat sämtlichen Aktiengesellschaften einen Gewinn von zusammen 478:306 $\mathscr{H}$ gebracht. Das gilt vom gesamten Geschäfte. Scheidet man bei den einzelnen Gesellschaften das Ergebnis der Vermögensverwaltung aus, so ermäßigt sich der Gewinn jener Gesellschaften auf $3443929 \mathscr{N}$. Die Gegenseitigkeitsvereine decken ilıren Verlust meist schon vor Abschluß der Rechnung aus der gesetzlichen Rücklage oder durch Nachschüsse (vgl. "Entwickelung a S. j0 u. 51); darüber hinaus haben drei Vereine für das Berichtsjalır einen Gewinn von zusammen $315779 \mathscr{M}$ und zwei Vereine einen Verlust von zusammen $5277 \mathscr{N}$ nachgewiesen.

Aus der Bilanz für I 9 I 6, die für die Aktiengesellschaften zusammen mit $46302753 \mathscr{M}$ und für die Gegenseitigkeitsvereine zusammen mit $20111719 \mathscr{M}$ abschließt, mögen folgende Posten angefülırt werden:

\begin{tabular}{|c|c|c|c|}
\hline Vermögenswerte & 4 Ahtiengesellschaften & 12 Gegenseitıgkeı & itsvereine \\
\hline Verpflichtungen der Alitionäre oder Garanten & $18117100 \mathscr{H}$ & - & $\mathscr{M}$ \\
\hline Kapitalanlagen .................. & 24488762 & 14349460 & . \\
\hline Forderungen $\ldots \ldots \ldots \ldots \ldots \ldots, \ldots$. & $3637 \mathrm{I} 65$ & 5657554 & . \\
\hline Kassenliestand $\ldots \ldots \ldots \ldots \ldots \ldots$ & 59726 & 100400 & $\cdot$ \\
\hline \multicolumn{4}{|l|}{ Verbundlichkerten } \\
\hline Aktıen- oder Garantiehapital . & $25528500 \mathscr{M}$ & - & $M$ \\
\hline Geset $r$ liche Ruchlage ............. & 2297099 . & 13243928 &. \\
\hline Sonderruichlagen $\ldots \ldots \ldots \ldots \ldots \ldots \ldots$ & 13237880 . & 2318527 & - \\
\hline Schadenruchlage $\ldots \ldots \ldots \ldots \ldots \ldots \ldots$. & 20292 & 780068 & - \\
\hline
\end{tabular}

Ubber die Kapitalanlagen gibt Tafel III 15 folgende Aufschlüsse. Von $1000 \mathscr{M}$ Kapitalanlagen kamen Ende I916 auf:

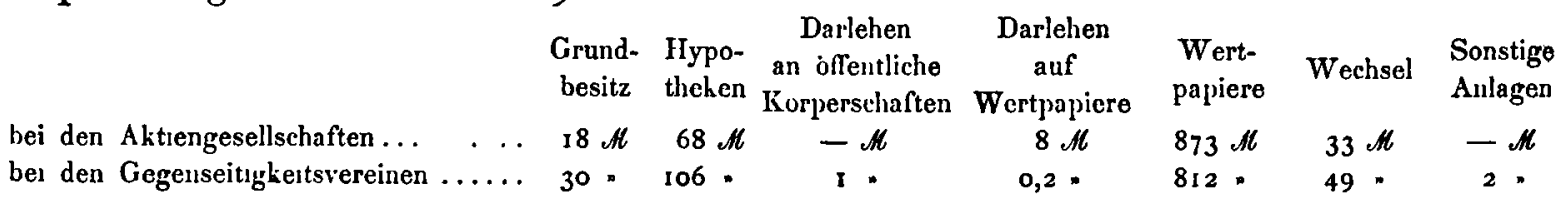

Im Vergleiche mit der Bilanz des Vorjahrs haben abgenommen der Grundbesitz um $103250 \mathscr{H}$ und der Iypothekenbestand um $3665 \overline{5} \mathscr{M}$, dagegen zugenommen der Buchwert der Wertpapiere um $5907884 \mathscr{M}$, während die Darlehen auf Wertpapiere sich nicht verïndert haben. 
Das aus.

lindische Geschn't

Uber die Bedeutung des ausländischen Geschäfts der deutschen Hagelversicherungsunternehmungen, das in den vorstehenden Zahlen überall eingeschlossen ist, sind folgende Angaben zu machen:

Im ganzen haben vier Aktiengesellschaften und vier Gegenseitigkeitsvereine auch im Auslande Gescläfte betrieben. In Ungarn und in Luxemburg war je eine Gesellschaft tätig, in Rußland arbeiteten drei, in den Niederlanden und in Österreich je vier Anstalten. Die Versicherungssumme belief sich auf 20,845 Millionen Mark oder 5,2 vom Tausend der von allen Unternelımungen überhaupt versicherten Summe; die durchschnittliche Höhe einer sellst abgeschlossenen ausländischen Versicherung stellte sich auf 13830 M. Die Rohbeitragseinnahme aus dem ausländischien Geschäfte betrug $332694 \mathscr{M}$ oder 7,1 vom Tausend der gesamten Rohbeiträge; die Schäden betrugen $406102 \mathscr{M}$ oder 12,2 vom Tausend der gesamten Schäden.

\section{Gruppe IV, Feuerversicherung (einschl. Versicherung mittelbarer Schäden) sowie die} Versicherungen gegen Sturmschäden, Wasserleitungsschäden und Einbruchdiebstahl

Die Versicherung mittilliarer Schalen

Dio Unter. nehmungen

Seitdem mit dem Inkraftreten des Versicherungsvertragsgesetzes die Schranken gefallen sind, welche bis dahin in dem größten Teile von Deutschland der Versicherung des durch den Eintritt eines Sachschadens entgehenden Gewinns, soweit hierbei die Feuerversicherung in Betracht kam, gezogen waren, haben die meisten der bei uns arbeitenden größeren Feucrversicherungsgesellschaften die Versicherung solcher mittelbarer Schäden in den Formen der Mietverlust-, der Betriebsverlust- und der Zuckerpreisdifferenzversicherung in ihren Geschäftsplan aufgenommen. Der Umfang dieser Geschäfte ist naturgemäß noch nicht beträchtlich, immerhin wird es von Wert sein, die Entwickelung von Anfang an statistisch verfolgen zu können; deshalb sind schon vom Jahre 19 I I ab einige Zusammenstellungen darüber mitgeteilt. Wegen des unmittelbaren Zusammenhangs dieser Versicherungen mit der Feuer- oder der Wasserleitungsschädenversicherung sind sie mit diesen Zweigen hier bei Gruppe IV behandelt. Es wird jedoch einstweilen nicht nötig sein, in diesem Texte näher auf sie einzugehen; der Hinweis auf die Tafeln wird genügen.

Von den deutschen Unternehmungen, die Versicherungen der Gruppe IV abschließen, unterstanden im Jahre 1916 der Reichsaufsicht 45 Aktiengesellschaften und 36 Gegenseitigkeitsvereine. Die zahlreichen Feuerversicherungsvereine auf Gegenseitigkeit, welche wegen der Beschrïnkung ihres Geschäftsbetriebs auf einen Bundesstaat der Aufsicht der Landesbehörden unterstehen oder welche gemäß $§ 3$ Abs. 2 des Versicherungsaufsichtsgesetzes der Landesaufsicht überwiesen worden sind und denen auch in ihrer Gesamtheit keine allzu große Bedeutung zukommt, können in dieser Statistik nicht behandelt werden, weil sie nicht an das Aufsichtsamt zu berichten brauchen.

Die Berlinische Feuer-Versicherungs-Anstalt hat ihren Geschäftsbetrieb auf die Zuckerpreisdifferenzversicherung ausgedehnt, während der Nordstern, Unfall- und Haftpflicht-Versicherungs-Aktien-Gesellschaft in Berlin-Schöneberg und der Nordstern, Feuer- 
Versicherungs-Aktien-Gesellschatti in Berlin-Schöneberg die Wasserleitungsschädenversicherung aufnahmen.

Die Union, Allgemeine Versicherungs-Aktien-Gesellschaft zu Berlin, die Schlesische Feuerversicherungs-Gesellschaft in Breslau und die Versicherungsgesellchaft Thuringia in Erfurt haben die Zuckerpreisdifferenzversicherung fallen lassen.

Die Hohenzollern, Versicherungs-Aktiengesellschaft in Cöln a. Rhein hat ihren Sitz nach Berlin und die Securitas, Versicherungs-Aktiengesellschaft in Berlin hat ihren Sitz nach Bremen verlegt.

Von den 45 Gesellschaften betrieben 40 die Feuerversicherung neben andern Zweigen, die übrigen 5 befaßten sich nicht mit der Feuerversicherung, sondern soweit die Versicherungszweige der Gruppe IV in Betracht kommen - nur mit einem oder melıreren der Nebenzweige, von denen die Einbruchdiebstahlversicherung der wichtigste ist.

Von den 36 unter Reichsaufsicht stehenden Feuerversicherungsvereinen auf Gegenseitigkeit sind in der vorliegenden Statistik 24 ausführlich behandelt. Die Feuer- und Einbruchdiebstahl-Versicherungsanstalt des Verbandes Deutscher Beamtenvereine a. G. in Berlin hat ihre Firma in Deutsche Beamten-Feuerversicherung auf Gegenseitigkeit, Anstalt des Verbandes Deutscher Beamtenvereine in Berlin und die Landwirtschaftliche Feuerversicherungs-Genossenschaft im Konigreich Sachsen zu Dresden hat ilıre Firma in Dresiner Feuerversicherung - Landwirtschaftliche Feuerversicherung in Königreich Sachsen - Allgemeine Feuerversicherung in Deutschland geändert. Von diesen 24 Vereinen beschränken sich nur $\tau$ auf die Feuerversicherung, 3 weitere betreiben daneben die Hagelversicherung; die übrigen arbeiten außer in der Feuerversicherung noch in dem einen oder andern der Nebenzweige der Sachversicherung. Die drei Vereine, die auch Hagelversicherungen abschließen, führen aber -- wie schon bei Gruppe III, S. 54* erwähnt - die verschiedenen Versicherungszweige vollstïndig voneinander getrennt, so daß hier nur auf das Feuer- und Einbruchdiebstallversicherungsgeschäft eingegangen $\mathrm{zu}$ werden braucht.

Hinsichtlich der ausländischen Versicherungsunternehmungen, die sich mit den zu Gruppe IV gehörigen Versicherungszweigen beschäftigen, ist zu bemerken, daß wie im vorigen Jahre die englischen, die französische und eine belgische fortgelassen worden sind. Der Hauptteil der Bestände dieser Gesellschaften ist durch Haftungs- und Ubergangsverträge bei deutschen Gesellschaften sichergestellt worden. Das Nähere hierüber findet sich in der Versicherungsstatistik für I914 (S. 60*), es erübrigt sich, hier darauf zurückzukommen.

Wir beschränken demnach unsern Bericht für I9I6 auf 15 ausländische Feuerversicherungsgesellschaften. Davon arbeiteten, soweit Versicherungszweige der Gruppe IV in Frage kommen, im Deutschen Reiche 2 nur in der Feuerversicherung; weitere 8 betrieben die Feuerversicherung neben andern Zweigen und die übrigen 5 befaßten sich nicht mit der Feuerversiclserung selbst, sondern nur mit den Nebenzweigen.

Bei den folgenden Ausführungen sowie bei der Betrachtung der Tafeln ist, soweit es sich um Feuerversicherung handelt, im Auge zu behalten, daßstets die Gesamtheit der Versicherungen dargestellt ist; eine Trennung nach Risiken konnte nirgends vorgenommen werden; ebensowenig hat die Hausratfeuerversicherung von der Liegenschaftsversicherung geschieden werden können. 
Uber den Umfang des Geschäfts der deutschen Unternehmungen in der Feuerversicherung Ende 19 5 und Fnde I916 gibt Tafel IV 1 Auskunft. Danach liefen an sellsst abgeschlossenen Versicherungen einschließlich der in Rückdeckung gegebenen 'Teile:

\section{Ende 1915}

bei den Aktrengesellschaften ..... . I 37849 I Vers. uber 128 gro Mill. At I 092048 Vers. uber 133326 Mill. $A$

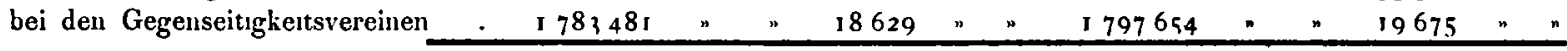
Zusammen .... 13161972 Vers. uber 147539 Mill. $\mathscr{M} \quad 12889702$ Vers. ubber 153001 Mill. $\mathscr{A l}$

Derim unmittelbaren Geschäfte durchschnittlich aufeineVersicherung entfallende Betrag hat sich, wie die folgende Zusammenstellung zeigt, in der letzten Zeit stärker wie bisher geändert.

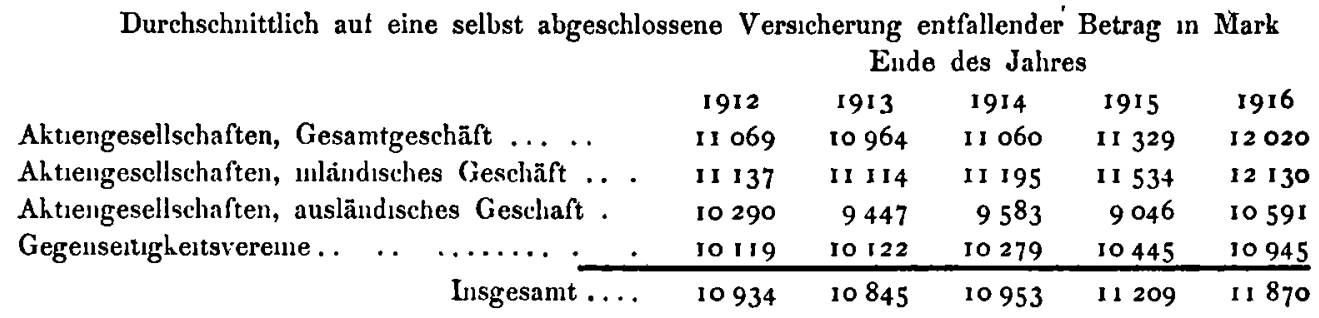

Die Alitiengesellschaften hatten außerdem noch Versicherungen in Höhe von 15074 Millionen Mark am Ende I9 5 und in IIöhe von 17187 Millionen Mark am Ende 1916 in Rückdeckung genommen, so daß die gesamte Versicherungssumme Ende I915 143984 und Ende 1916 150512 Millionen Mark betrug. In dieser Summe sind alle diejenigen Betrïge doppelt entlıalten, welche von einer Gescllschaft, die in dieser Statistik behandelt wird, versichert und bei einer ebensolchen Gesellschaft in Rückdeckung gegeben sind. Dasselbe gilt von den unten angeführten Zahlen über die Gesamtversicherungssumme bei den andern Versicherungszweigen. Der Anteil des mittelbaren Geschüfts am Gesamtgeschäfte beträgt 11,4 v. $\mathrm{H}$. Von den Gegenseitigkeitsvereinen haben zwei den geringen Betrag von zusammen 50 Millionen Mark in Rückdeckung übernommen.

Rechnet man zur Feucrversicherung die Versicherung mittelbarer Schäden (Mietverlust-, Betriebsverlust- und Zuckerpreisdifferenzversicherung) hinz.u, so belief sich die gesamte Versicherungssumme Ende I 916 bei den Aktiengesellschaften auf 150918 und bei den Gegenseitigkeitsvereinen auf 19733 Millionen Mark. Der davon für eigene Rechnung behaltene Teil stellte sich:

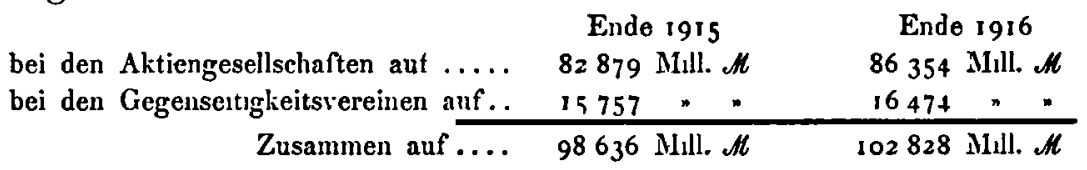

und betrug in Tausendsteln der überhaupt versicherten Summen:

$\begin{array}{lll}\text { bei den Aktiengesellschaften ..... } & 575 & 572 \\ \text { bei den Gegenscitigheitsı ereinen . } & 842 & 835\end{array}$

Die Tafel IV 2 behandelt lediglich das deutsche Feuerversicherungsgeschäft der deutschen Aktiengesellschaften; aus dieser Tafel in Verbindung mit Tafel IV 1 ergibt sich folgende 'Teilung des Geschäfts:

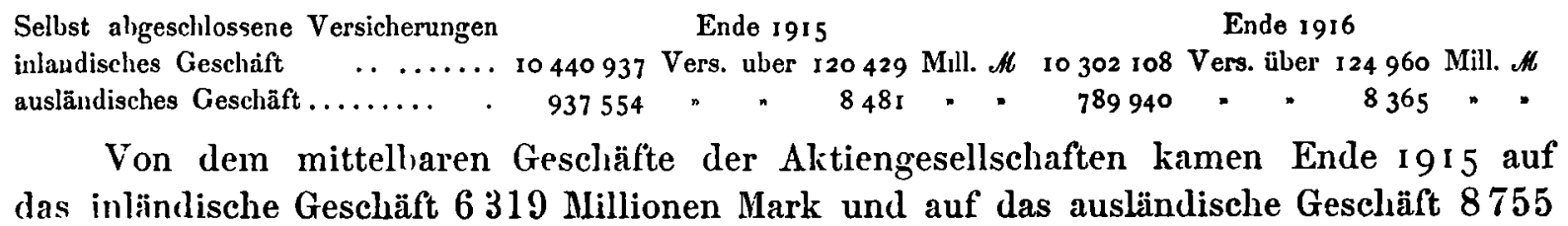


Millionen Mtark, Ende 1916 auf das inländische Geschäft 7864 Millionen Mark und auf das ausländische Geschïft 9323 Millionen Mark.

Das gesamte inländische Feuerversicherungsgeschäft der Aktiengesellschaften erstreckte sich Fnde 1915 über 126748 Millionen Mark und Ende I916 über 1:32 824 Millionen Mark; das gesamte ausländische Geschüft bezifferte sich Ende 19 I 5 auf 17236 Millionen Mark und Ende igr6 auf 17688 Millionen Mark. Der Anteil der mittelbaren Versicherungen an der Gesamtheit betrug danach beim inländischen Geschäft I9 I5 4,9 v. H. und I 9 I 6 5,9 v. II., beim ausländischen dagegen I 915 50,8 v. H. und I 916 52,7 v. H.

Von den Gegenseitigkeitsvereinen haben nur drei (der Versicherungsverein Deutscher Eisenbahnbediensteten, die Deutsche Beamten-Feuerversicherung auf Gegenseitigkeit, Anstalt des Verbandes dcutscher Beamtenvereine, und die Gothacr Feuerversicherungsbank) ausländische Geschäfte, und zwar in geringem Maße, abgeschlossen. Alle zusammen hatten Ende I9 5 im Ausland 1999 Versicherungen über 45 Millionen Mark und Ende I9ı 2016 Versicherungen über 41 Millionen Mark in Kraft.

Uber den Umfang der Mietverlust-, der Betriebsverlust- und der Zuckerpreisdifferenzversicherung geben die Tafeln IV 3 bis 7 Auskunft.

In der Sturmschädenversicherung haben im Jahre 1916 dieselben beiden Aktiengesellschaften gearbeitet wie in den Vorjahren; der Erfolg ist auch in diesem Jahre kein großer gewesen. Nach Tafel IV 8 liefen nämlich Encle 1915 insgesamt 2525 Versicherungen über 224,5 Millionen Mark und Ende 19162739 Versicherungen mit 224,6 Millionen Mark, so daß die Zahl der Versicherungen um 214 und die versicherten Summen um 0,1 Millionen Mark zugenommen haben. Im Auslande wurde dieser Zweig nicht betrieben. Für eigene Rechnung wurde nur der geringe Betrag von 45 Millionen Mark behalten. Rückversicherungen wurden von diesen beiden Gesellschaften nicht übernommen.

In der Wasserleitungsschädenversicherung haben Ende 191629 deutsche Aktiengesellschaften gearbcitet. Neu hinzugekommen sind der Nordstern, Unfall- und Ilaftpflicht-Versicherungs-Aktien-Gesellschaft in Berlin-Schöneberg und der Nordstern, Feuer-Versicherungs-Aktien-Gesellschaft in Berlin-Schöneberg.

Nach Tafel IV 9 liefen an selbst abgeschlossenen Versicherungen Ende 19I5 136630 Versicherungen über 6176 Millionen Mark und Ende 1916 1316568 Versicherungen über 6414 Millionen Mark, was einen Abgang von 62 Versicherungen und einen Zugang von 238 Millionen Mark Versicherungssumme bedeutet.

Die Gesellschaften hatten außerdem noch Versicherungen in IIöhe von 213,0 Millionen Mark zu Ende 1915 und in Höhe von 224,8 Millionen Mark zu Ende 1916 in Rückdeckung genommen, so daß die gesamte Versicherungssumme Ende I916 6639 Millionen Mark betrug. Von den Versicherungssummen kamen Ende 19 I 5 und Ende I916 96,0 v. H. auf das deutsche Geschält. Der weitaus größte Teil der Versicherungen, nämlich 5737 Millionen, wurde für eigene Rechnung behalten.

Von den Gegenseitigkeitsvereinen ist im Berichtsjahre diese Versicherungsart nur von der Concordia, Hannoversche Feuer-Versicherungs-Gesellschaft, betrieben worden, und zwar nur unmittelbar und nur innerhalb des Deutschen Reichs. Das gesamte Geschäft umfaßte am Ende des Berichtsjahrs 71 Versicherungen über $427 j 000 \mathscr{M}$.

Sehr viel bedeutender ist das Geschält in der Einbruchdiebstahlversicherung. Es haben in diesem Zweige Ende 191643 Aktiengesellschaften gearbeitet. 
Von den selbst abgeschlossenen Versicherungen der deutschen Aktiengesellschaften kamen:

\begin{tabular}{|c|c|c|c|c|c|c|c|c|c|c|}
\hline \multirow{3}{*}{$\begin{array}{l}\text { auf das inländısche Geschäft .... } \\
\text { auf das auslandısche Geschaft . . }\end{array}$} & \multicolumn{5}{|c|}{ Ende 1915} & \multicolumn{5}{|c|}{ Ende 1916} \\
\hline & 1114136 & Vers & uber & $145^{26}$ & Mill. $\mathscr{A}$ & I 124792 & Vers. & uber & 14964 & Mill. $\mathscr{H}$ \\
\hline & 63091 & $n$ & $n$ & 1328 & $n$ & $63 \times 56$ & $n$ & $n$ & I 303 & n \\
\hline
\end{tabular}

Das unmittelbare inländische Geschäft hat demnach um 10656 Versicherungen und 438 Millionen Mark zugenommen, das ausländische um 65 Versicherungen zugenommen und 25 Millionen Mark abgenommen. In Rückdeckung übernommen hatten diese Gesellschaften außerdem im inländischen Betrieb am Ende des Vorjahrs 2 664 und am Ende des Berichtsjahrs 2953 Millionen Mark, so daß die gesamte Versicherungssumme des deutschen Geschäfts Ende I9 I5 17190 Millionen Mark und Ende I9 617917 Millionen Mark betrug. Im ausländischen Geschäfte waren in Rückdeckung genommen Ende 19 I 51541 und Ende 1916 1257 Millionen Mark; demnach ist die gesamte Versicherungssumme des ausländischen Geschälts von 2869 auf 2560 Millionen Mark zurückgegangen. Der Anteil des mittelbaren Geschüfts am Gesamtgeschäft in der Einbruchdiebstahlversicherung belief sich danach beim inländischen Geschäft Ende I 9 I 5 auf 15,5 und Ende 1916 auf 16,5 v. H., beim ausländischen Geschäft auf $\overline{\mathbf{3}} 3,7$ bezw. 49,1 v. $\mathrm{H}$.

Von den Gegenseitigkeitsvereinen haben in der Einbruchdiebstahlversicherung im Berichtsjahre 13 gearbeitet. Das Geschäft war auch im Jahre 1916 noch gering, es stellte sich Ende des Jahres auf insgesamt 9!) 147 Versicherungsscheine über 1035 Mullionen Mark. Es handelt sich lediglich um unmittelbare deutsche Versicherungen.

Alle 56 Unternehmungen zusammen hatten Finde I916 Versicherungen in Höhe von 21512 Millionen Mark in Kraft, davon liefen 14840 Millionen für eigene Rechnung.

Bei den ausländischen Versicherungsunternehmungen sehen wir, wie bereits oben erwähnt, von den englischen Gesellschaften, sowie von der französischen Gesellschaft und von der Belgischen Allgemeinen Feuer-Versicherungs-Aktien-Gesellschaft in Brüssel ab und berichten nur über Io Gesellschaften, wobei wir uns wie bisher auf deren deutsches Geschäft beschränken.

Diese Gesellschaften hatten im Deutschen Reiche selbst abgeschlossene Feuerversicherungen Ende I 9 I 5 in IIöhe von 5612 Millionen Mark und Ende I 9 I 6 in IIöhe von 5680 Millionen Mark; in Rückdeckung waren zu denselben Zeitpunkten Versicherungen im Betrage von 291 und von 376 Millionen Mark übernommen; der gesamte Versicherungsbestand betrug also in der Feuerversicherung am Schlusse des Vorjahrs 5 !02 und am Schlusse des Geschäftsjahrs 6056 Millionen Mark. Im Jahre 1916 baben die selbst abgeschlossenen Versicherungen um 69 Millionen und die in Rückdeckung übernommenen Versicherungen um 85 Millionen Mark zugenommen.

Der Anteil des mittelbaren Geschäfts am Gesamtgeschäft ist von 4,9 v. H. auf 6,2 v. H. gestiegen.

UUber das geringfügige deutsche Geschäft dieser Gesellschaften in der Versicherung mittelbarer Scliäden unterrichten die Tafeln IV 14 und 15.

Von den ausländischen Gesellschaften, die im Deutschen Reiche in der Wasserleitungsschädenversicherung gearbeitet haben, kommen hier nur vier in Betracht. 
Neu hinzugekommen ist die Schweizcrische National-Versicherungs-Gesellschaft in Basel. Es wurden nur unmittelbare Versicherungen abgeschlossen. Das Geschüft umfaßte am Ende des Berichtsjahırs 677 Versicherungen über 19 Millionen Mark, gegenüber 586 Versicherungen über 15 Millionen Mark am Ende des Vorjahrs.

In der Einbruch di ebstahlversicherung berichten wir über dreizehn ausländische Gesellschaften. Neu hinzugekommen ist der Lloyd Belge, Versicherungs-Aktien-Gesellschaft in Antwerpen. Von diesen haben neun lediglich unmittelbare, vier auch mittelbare Geschäfte betrieben. Nach Tafel IV 17 waren an selbst abgeschlossenen Versicherungen Ende 1915 732 Millionen Mlark und Ende 1916 789 Millionen Mark in Kraft. Rechnet man die in Rückdeckung übernommenen Versicherungen hinzu, so erlıält man für den Bestànd des deutschen Geschäfts am Schlusse des Vorjahrs 833 und für den Bestand am Schlusse des Berichtsjalırs 898 Millionen Mark.

Der Wert aller im Deutschen Reiche gegen Feuerschaden versicherten Gegenstände, wie er sich aus der Summe aller im Reiche unmittelbar versicherten Beträge ergibt, kann aus den vorstehenden Angaben allein nicht entnommen werden. Abgesehen von den verhältnismäßig kleinen Summen, die bei den kleinen Gegenseitigkeitsvereinen und bei den unter Landesaufsicht stehenden privaten Unternehmungen versichert sind, fehlen noch die Versicherungen von 48 öffentlichen Feuerversicherungs-Sozietäten. Uber den Umfang dieser Versicherungen veröffentlicht der Verhand der öffentlichen Feuerversicherungs-Anstalten jährlich in den "Mitteilungen für die öffentlichen Feuerversicherungs-Anstalten “ Zusammenstellungen, deren Zuverlässigkeit nicht zu bezweifeln ist. Mit llilfe dieser Angaben kann man den gesamten Feuerversicherungsbestand im Deutschen Reiche annähernd folgendermaßen feststellen:

Es waren an deutschen Werten versichert in Millionen Mark:

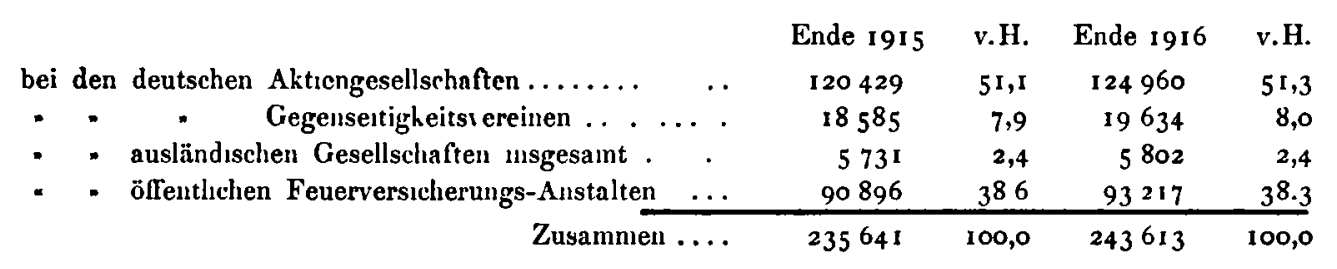

Die Rohbeitragseinnahme im Berichtsjahr und die im Berichtsjahr entstandenen Schälen aller Arten von Risiken, jedoch olıne Schadenermittelungskosten, sind für die deutschen privaten Unternelımungen in den Tafeln IV 18 bis 24 und für das deutsche Geschäft der ausländischen Gesellschaften in den Tafeln IV 25 bis 29 zusammengestellt.

In der Feuerversicherung haben danach im Jahre 191640 deutsche Aktiengesellschaften $270917937 \mathscr{M}$ und 24 Gegenseitigkeitsvereine $47281115 \mathscr{M}$, alle diese Unternehmungen zusammen $318199052 \mathscr{M}$ an Beiträgen eingenommen; in diesem Jahre sind Schäden (einschließlich des Anteils der Rückversicherer, aber ohne Schadenermittelungskosten) bei den Aktiengesellschaften im Betrage von $136965081 \mathscr{M}$, bei den Gegenseitigkeitsvereinen im Betrage von $9014896 \mathscr{M}$, bei allen Anstalten zusammen im Betrage von $145979977 \mathscr{M}$ entstanden. Dabei ist zu beachten, daß einige Gegenseitigkeitsvereine, um Nachschüsse zu vermeiden, Vorbeiträge erheben, die den 
regelmäßigen Bedarf weit übersteigen und den daraus entstehenden Uberschuß den Versicherten wieder zufülıren (vgl. Tafel IV 40).

Der durchschnittliche Betrag eines Schadens aus selbst ahgeschlossenen Feuerversicherungen (ohne Ermittelungskosten) stellte sich bei den Aktiengesellschaften im inländischen Geschäft auf $590 \mathscr{H}$, im ausländischen Geschäft auf $870 \mathscr{A}$ gegen $586 \mathscr{A}$ bezw. $816 \mathscr{M l}$ im Vorjahre.

Bei den Gegenseitigkeitsvereinen hat der Schadenfall aus selbst abgeschlossenen Versicherungen im inländischen Geschäfte durchschnittlich $471 \mathscr{M}$, im ausländischen Geschäfte (21 Fälle) durchschnittlich $109 \mathscr{M}$ erfordert.

Über die Versicherung mittelbarer Schäden fülıren die Tafeln 19 bis 21 zu folgenden Ergebnissen:

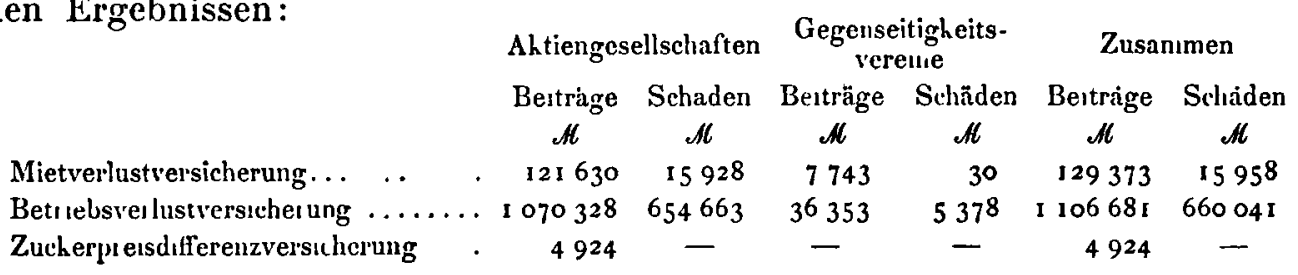

Die beiden Gesellschaften, die in der Versicherung gegen Sturmschäden gearbeitet haben, erzielten (Tafel IV 22) aus diesem Geschäft eine Beitragscinnalıme von $179055 \mathscr{M}$, welche nur aus dem deutschen Betriebe herrührte. An Schäden sind aus selbst abgeschlossenen Versicherungen 449 mit $79011 \mathscr{A}$ (ohne Ermittelungskosten) zu verzeichnen gewesen; der durchschnittliche Betrag eines Schadens stellte sich demnach auf $176 \mathscr{A}$.

Die neunundzwanzig Aktiengesellschaften, die in der Versicherung gegen Wasserleitungsschäden gearbeitet haben, erziclten aus diesem Geschäfte (Tafel IV 23) eine Beitragseinnahme von $3301876 \mathscr{A}$. An Schäden sind $1193711 \mathscr{H}$ (olne Ermittelungskosten) zu verzeichnen gewesen. Der durchschnittliche Betrag eines Schadens aus selbst abgeschlossenen Versicherungen stellte sich beim inländischen Geschäft auf $64 \mathscr{A l}$, beim auslaindischen Geschält auf $49 \mathscr{N}$.

In der Einbruchdiebstahlversicherung (Tafel IV 24) haben 43 deutsche Aktiengesellschaften $16928358 \mathscr{A}$ an Beiträgen eingenommen und $6703020 \mathscr{A}$ für Schäden verausgabt; die 13 andern Unternelımungen haben $842403 \mathscr{M}$ Beiträge eingezogen und $24377 \mathscr{M}$ für Schäılen gezahlt.

Der durchschnittliche Betrag eines Schadens aus den selbst abgeschlossenen Versicherungen stellte sich bei den Aktiengesellschaften (olnne Ermittelungskosten) im inländischen Geschäft auf $246 \mathscr{M}$ und im ausländischen Geschäft auf $286 \mathscr{A}$, bei den Gegenseitigkeitsvereinen auf $14 \mathscr{N}$.

Für das deutsche Feuerversicherungsgeschäft der berücksichtigten 10 ausländischen Gesellschaften gelten folgende Zahlen ('Tafel IV 25). Die Beitragseinnahme belief sich auf $11275661 \mathscr{M}$; für Sclı̈lden waren $4578311 \mathscr{H}$ auf $\iota$ wwenden. Von den Beiträgen stammten 93,3 v. H. aus selbst abgeschlossenen Versicherungen und $6,7 \mathrm{v} . \mathrm{H}$. aus Rückversicherungen, von den Schäden betrafon 92,4 v. H. selbst abgeschlossene Versicherungen und 7,6 v. H. in Rückleckung übernommene Versicherungen.

Für die Versicherung mittelharer Schälen lıaben die ausländischen Gesellschaften in Deutschlland insgesamt $10579 \mathscr{M}$ an Beiträgen eingenommen und $4331 \mathscr{M}$ für Schäden ausbezahlt. 
Für das deutsche Wasserleitungsschädenversicherungsgeschäft der vier ausländischen Gesellschaften hat die Beitragseinnahme $13769 \mathscr{M}$ betragen; für Schäden sind $2979 \mathscr{N}$ gezahlt worden.

Das deutsche Einbruchdiebstahlversicherungsgeschäft der hier in Betracht kommenden 13 ausländischen Gesellschaften, das im wesentlichen unmittelbar betrieben wird, zeigt sich auch hinsichtlich der Beitragseinnahme und der Schadenleistungen (Tafel IV 29) als wenig bedeutend. Die Bcitragseinnahme lat nur $1015559 \mathscr{M}$ betragen, für Schäden sind $512568 \mathscr{M}$ zu zahlen gewesen.

Wie oben die Versicherungssummen annähernd für das gesamte unmittelbare deutsche Feuerversicherungsgeschäft ermittelt wurden, so können mit Hilfe der vorstehenden Zahlen und der Angaben in den "Mitteilungen für die öffentlichen FeuerversicherungsAnstalten " auch die Schadenbeträge. die den deutschen Geschädigten zugeflossen sind, angenähert festgestellt werden.

\begin{tabular}{|c|c|c|c|c|}
\hline \multirow[t]{3}{*}{ 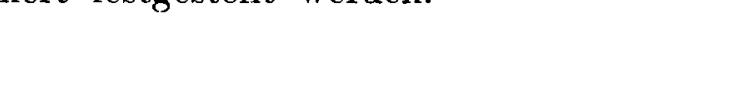 } & \multicolumn{4}{|c|}{ Schadenzahlungen } \\
\hline & \multicolumn{2}{|c|}{ Inı Jahre 19I5 } & \multicolumn{2}{|c|}{ in Jahre 1916} \\
\hline & $1000 \mathscr{N}$ & v II & $1000 \mathscr{A}$ & v H \\
\hline deutsche Aktiengesellschaften & 93410 & 58,9 & 83286 & 60,8 \\
\hline " Gegensertigkeitsvereme .... . & 10533 & 6,6 & 8996 & 6,5 \\
\hline $\begin{array}{l}\text { auslandische Gesellschaften mit uber } 100000 \mathscr{N} \text { Bel- } \\
\text { tragsemnahme aus dem deutschen Geschaft } . .\end{array}$ & 5477 & 34 & +231 & $3, \mathrm{I}$ \\
\hline $\begin{array}{l}\text { auslandische Gesellschaften mit unter } 100000 \mathscr{H} \text { Be1- } \\
\text { tragseinnahme aus dem deutschen Geschaft } .\end{array}$ & 89 & 01 & 70 & $0, \mathbf{I}$ \\
\hline deutsche offentliche Fener ersicherungs-Anstalten & $49 \times 15$ & $3 r, 0$ & 40433 & 295 \\
\hline Zusammen & I 58624 & 100,0 & 137016 & 100,0 \\
\hline
\end{tabular}

In diesen Zahlen kommt der Utbergang der meisten deutschen Versicherungen hei englischen Gesellschaften auf deutsche Aktiengesellschaften deutlich zum Ausdruck

Die Beitragseinnahme ist, wie die folgende Zusammenstellung zeigt, um 0,3 Millionen Mark und die Zahlungen für ausländische Schäden sind um 1 Million Mark größer gewesen als 1915.

\begin{tabular}{|c|c|c|c|c|c|}
\hline & & \multicolumn{2}{|c|}{$\begin{array}{l}\text { Ausländısches Geschaft der } \\
\text { deutschen Gesellschaften }\end{array}$} & \multicolumn{2}{|c|}{$\begin{array}{l}\text { Deutsches Geschaft der aus } \\
\text { landıschen Gesellschaften }\end{array}$} \\
\hline & & Beitrage & Schaden & Betträge & Schåden \\
\hline & & $\mathscr{M}$ & $\mathscr{M}$ & $\mathscr{M}$ & $\mathscr{M}$ \\
\hline Feuerversicherung ...... & . & 63234980 & 39453446 & II 27566 I & 45783 I I \\
\hline Mietverlustversicherung,$\ldots \ldots$ & $\cdot$ & 3229 & I & 2624 & 307 \\
\hline Betriebsverlustversicherung ... . . . & $\ldots$ & 813060 & 375875 & 7955 & 4024 \\
\hline Zuckerpreisdıfferenzversıcherung. . . & $\ldots \ldots$ & 一 & $\cdots$ & - & - \\
\hline Sturmschadenversicherung & . & - & - & - & - \\
\hline Wasserleitungsschädenversıcherung ... & . $\ldots$ & I 10915 & 27699 & 13769 & 2979 \\
\hline \multirow[t]{2}{*}{ Embruchdiebstahlversicher ung } & . & 1485389 & 453214 & 1015559 & 512568 \\
\hline & mmen . & 65647573 & 40310235 & $123155^{68}$ & $5098 \div 89$ \\
\hline
\end{tabular}

Das Ruslandische Geschuft der deutschen und das deutsche Geschăft der ausiandischen Gesellsehaften

Im Jahre I9 6 haben, um nur diejenigen Länder zu nennen, in denen deutsche Gesellschaften besonders zahlreich vertreten sind, in der Feuerversicherung gearbeitet in den Niederlanden 30, in Belgien 24, in der Schweiz 19, in Österreich 14, in Dänemark 13, 
in Luxemburg und in Skandinavien je 12, in der Türkei 10, in China, in Siam und in Mexiko je 10, in Norwegen und in Chile je 7, in Brasilien und auf den Philippinen je 6 usw. -- Die Nebenzweige der Feuerversicherung haben naturgemäß erst weniger weite Verbreitung gefunden; doch arbeiteten in der Einbruchdiebstahlversicherung im Jahre 19 I 6 in der Schweiz bereits 20, in den Niederlanden 16, in Belgien 10, in Österreich 9, in Dänemark 6, in Luxemburg und in Schweden je 5 usw.; Wasserleitungsschädenversicherung haben in Dänemark und in der Schweiz je 7, in Österreich und in den Niederlanden je 4, in Schweden 2, in Belgien und in Ungarn je eine deutsche Gesellschaft betrieben.

Die Betricbs. Einnahmen und

- Ausgahen und der Betriebs-

Gewina ites

Berichtsjahrs

Die Feuerversicherungs-Aktiengescllschaften arbeiten außcr auf dem Gebiete der Feuerversicherung meistens auch noch in anderen Versicherungszweigen, ohne daß sie in ihren Büchern die sämtlichen Einnahme- und Ausgabeposten streng nach Geschäftszweigen trennteu. Es ist jedoch seit einer Reihe von Jahren angängig, die Verwaltungskosten vollständig auf die einzelnen Zweige aufzuteilen, so daß die Betriebs-Einnahmen den Betriebs-Ausgaben nehst Verwaltungskosten und Steuern gegenübergestellt und somit der aus dem Versicherungsgeschäft allein herrührende Betriebs-Gewinn des Berichtsjahrs hergeleitet werden kann (Tafel IV 30 bis 33). Der Gewinn aus dem Gesamtgeschä fte wird in der Bilanz (Tafel IV 38) nachgewiesen

Der Gleıchformigkeit wegen sind die Aktiengesellschaften und die Gegenseitigkeitsvereine, die nur einen Geschäftszweig pflegen, ebenso behandelt worden.

Bei Gruppe IV sind als Betriebs-Einnahmen die Beiträge des Geschäftsjahrs für eigene Rechnung (Jahreseinnahme nach Abzug der Ristorni und der verausgabten Rückversicherungsbeiträge), die Nebenleistungen der Versicherten, die Frsparnis aus der Schadenrücklage des Vorjahı's und die Abnahme der Pı̈̈mienüberträge zusammengefaßt; als Betriebs-Ausgaben kommen die Zahlungen und Rückstellungen für Schälen, die im Geschäftsjahr entstanden sind (nach Alızug der Leistungen der Rückversicherer, aber einschließlich der Schadenermittelungskosten), die Leistungen für gemeinnützige Zwecke, der Zuschuß zur Schadenrücklage des Vorjahrs und die Zunahme der Prämienüberträge in Betracht.

Bei den deutschen Unternehmungen betrugen in der Feuerversicherung einschließlich Mietverlust-, Betriebsverlust- und Zuckerpreisdifferenzversicherung:

\begin{tabular}{|c|c|c|c|c|}
\hline & & $\begin{array}{l}\text { bel der Gesamtheit } \\
\text { der Unternehmungen }\end{array}$ & $\begin{array}{l}\text { bel } 40 \text { Alitien- } \\
\text { gesellschaften }\end{array}$ & $\begin{array}{l}\text { bei } 24 \text { Gegen- } \\
\text { seitıgkeitsveremen }\end{array}$ \\
\hline die Summe der & Betriebs-Emnahmen & $197746318 \mathscr{H}$ & $154989957 \mathscr{K}$ & $4275^{6} 361 \mathscr{A}$ \\
\hline ” & Betriebs-Ausgaben & 91972569 & 83028457 & 8944112 \\
\hline
\end{tabular}

Von je $1000 \mathscr{M}$ dieser Betriebs-Einnahmen kamen:

\begin{tabular}{|c|c|c|}
\hline $\begin{array}{l}\text { bet der Gesamtheit } \\
\text { der Unternehmungen }\end{array}$ & $\begin{array}{l}\text { bei den } 40 \text { Aktien - } \\
\text { gesellschaften }\end{array}$ & $\begin{array}{l}\text { bel den } 24 \text { Gegen- } \\
\text { seltigkeitsvereinen }\end{array}$ \\
\hline $918 \mathscr{K}$ & $902 \mathscr{N}$ & $979 \mathscr{M}$ \\
\hline 9 " & $8 \%$ & $13 *$ \\
\hline $70 "$ & $87 n$ & 8. \\
\hline $3 "$ & $3 "$ & $-\infty$ \\
\hline $1000 \mathscr{N}$ & $1000 \mathscr{M}$ & $1000 \mathscr{H}$ \\
\hline
\end{tabular}

auf Reunbeiträge .... ...........

" Nebenlesstungen der Versicherten ...

- Ersparms aus der Schadenruchlage..

* Abnahme der Pramenübertrage ... 
Von je 1000 eth der Betriebs-Ausgaben kamen:

\begin{tabular}{|c|c|c|c|}
\hline & $\begin{array}{l}\text { bei der Gesantheit } \\
\text { der Unternehmungen }\end{array}$ & $\begin{array}{l}\text { bel den } 40 \text { Ahtien- } \\
\text { gesellschaften }\end{array}$ & $\begin{array}{l}\text { bel den } 24 \text { Gegen- } \\
\text { seitigkeitsveremen }\end{array}$ \\
\hline auf gezahlte Schaden & $483 \mathscr{K}$ & $473 \mathscr{N}$ & $578 \mathscr{A}$ \\
\hline " zurückgestellte Schảden & $382 "$ & 397 & 24 I $"$ \\
\hline "Letstungen für gem Zwecke. & 33 & $33 "$ & $33 "$ \\
\hline "Zuschuß zur Schadenruchlage & $2 "$ & $3 "$ & $-"$ \\
\hline \multirow[t]{2}{*}{ "Zunahme der Prämıenuberträge } & I $00 "$ & $94 "$ & $148 n$ \\
\hline & $1000 \mathscr{A}$ & I $000 \mathscr{H}$ & $1000 \mathscr{N}$ \\
\hline
\end{tabular}

Von den im Geschäftsjahr entstandenen Schadensummen wurden durchschnittlich bei den Aktiengesellschaften 54,4 v. H., bei den Gegenseitigkeitsvereinen 70,5 v. H. und bei allen Anstalten zusammen 55,9 v. H. noch im Geschäftsjahre gezahlt. Der Rest wurde der Schadenrücklage überwiesen. -- Von den Ieistungen für gemeinnützige Zwecke beruhten bei den Aktiengesellschaften 66,4 v. H. und bei den Gegenseitigkeitsvereinen 92,8 v. H. auf gesetzlicher Vorschrift.

An Verwaltungskosten und Steuern hatten für die Feuerversicherung die 40 Aktiengesellschaften $49684500 \mathscr{M}$ und die 24 Gegenseitigkeitsvereine $7310761 \mathscr{M}$ aufzuwenden.

Einen Verlust aus dem Betriebe der Feuerversicherung des Berichtsjahrs von zusammen $237147 \mathscr{N}$ haben 5 Aktiengesellschaften nachgewiesen; den übrigen Gesellschaften hat das Berichtsjahr einen Gewinn von insgesamt $22514147 \mathscr{M}$ gebracht.

Von den Gegenseitigkeitsvereinen hat nur einer einen Verlust, und zwar von $3004 \mathscr{M}$; die übrigen hatten einen Gewinn von insgesamt $26504492 \mathcal{A}$. Zu dieser Zahl ist jedoch zu bemerken, daß davon allein $18547708 \mathscr{M}$ auf die Gothaer Feuerversicherungsbank und $4066232 \mathscr{M}$ auf die Württembergische Feuerversicherung kommen. Diese Ubberschüsse sind kein Gewinn im gewöhnlichen Sinne; sıe haben vielmehr darin ihren Grund, daß die genannten Unternehmungen Beiträge erheben, die den voraussichtlichen Bedarf weit übersteigen, und den Uberschuß den Versicherten dann im folgenden Jahre zurückerstatten (vgl. S. 66* und "Entwickelung" S. 50).

Zu der Sturmschäden-, der Wasserleitungsschäden- und der Einbruchdiebstahlversicherung ist hier folgendes $\mathrm{zu}$ bemerken:

Es betrug

die Summe der Betriebs-Eunnahmen

die Sumne der Betriebs-Einnahmen

\begin{tabular}{|c|c|c|}
\hline \multirow{2}{*}{$\begin{array}{c}\text { bei der } \\
\text { Sturmschaden- } \\
\text { versicherung } \\
\text { (2 Aktien- } \\
\text { gesellschaften) }\end{array}$} & \multicolumn{2}{|c|}{$\begin{array}{l}\text { bei der Wasserleitungsschaden- } \\
\text { versicherung }\end{array}$} \\
\hline & $\begin{array}{l}\text { (29 Aktien- } \\
\text { gesellschaften) }\end{array}$ & $\begin{array}{l}\text { (1 Gegen- } \\
\text { seitigheits- } \\
\text { rei em) }\end{array}$ \\
\hline $42755 \mathscr{A}$ & $3075460 \mathscr{M}$ & $578 \mathscr{M}$ \\
\hline 22885 & I 2 I 3749 " & 86 \\
\hline
\end{tabular}

bet der Einbruchdiebstahlversicherung

(43 Aktien- (13 Gegenseitiggesellschaften) beitsves eine)

$13501957 \AA$ 6762436
$741351 \mu$ 352 I $24 \cdots$

Von je $1000 \mathscr{A}$ der Betriebs-Einnahmen kamen:

\begin{tabular}{|c|c|c|c|c|c|c|c|c|c|}
\hline auf Rembeitrage..... & $879 \mathscr{H}$ & 915 & & 997 & & 844 & & 966 & $\mathscr{H}$ \\
\hline "Nebenleistungen der Versicherten & I" & 4 & $"$ & 3 & $"$ & 12 & $"$ & 18 & $"$ \\
\hline "Ersparnis aus der Schadenrucklage & $40 "$ & 79 & $"$ & - & $"$ & I 37 & n & I 5 & $"$ \\
\hline " Abnahme der Prãnuenubertrage & $80 "$ & 2 & $n$ & - & 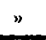 & 7 & $n$ & I & " \\
\hline
\end{tabular}

Von je $1000 \mathscr{M}$ der Betriebs-Ausgaben kamen:

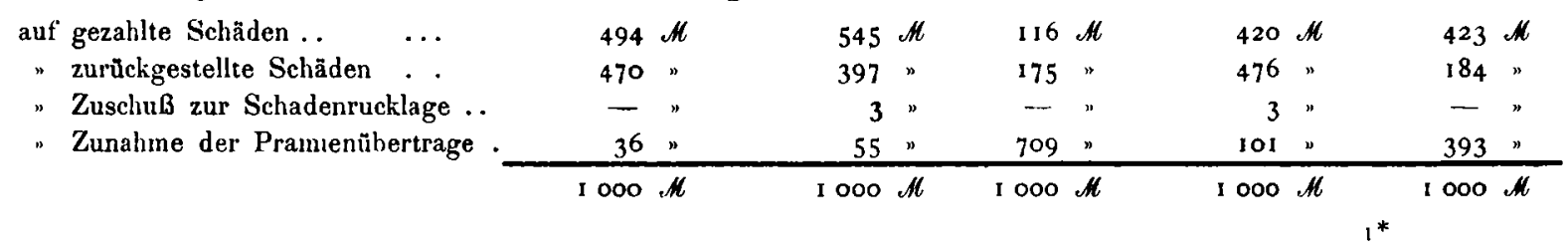


Von den im Geschäftøjahr entstandenen Schäden für eigene Rechnung wurden bei der Sturmschädenversicherung 51,3 v. II., bei der Wasserleitungsschädenversicherung 57,8 v. H. und bei der Einbruchdiebstahlversicherung 47,6 v. H. noch im Geschäftsjalıre beglichen, der Rest wurde in Schadenrücklage gestellt.

An Verwaltungskosten und Steuern hat die Sturmschädenversicherung $16709 \mathscr{M}$, die Wasserleitungsschädenversicherung bei den Aktiengesellschaften $1026942 \mathscr{M}$, bei 1 Gegenseitigkeitsverein $308 \mathscr{A}$ und die Versicherung gegen Einbruchdiebstahl bei den Aktiengesellschaften $4166093 \mathscr{N}$ und bei den Gegenseitigkeitsvereinen $191723 \mathscr{H}$ erfordert. In der Sturmschädenversicherung haben beide Aktiengesellschaften einen Gewinn von 31 i1 $\mathscr{A}$. In der Wasserleitungsschädenversicherung haben 4 Aktiengesellschaften einen Verlust von $19800 \mathscr{M}$ und 25 Aktiengesellschaften und 1 Gegenseitigkeitsverein einen Gewinn von $854569 \mathscr{H}$ bezw. $184 \mathscr{A}$ gehabt. In der Einbruchdiebstahlversicherung haben 32 Aktiengesellschaften einen Gewinn von $2687792 \mathscr{M}$ und 11 Aktiengesellschaften einen Verlust von $114364 \mathscr{N}$ gehabt. Das kleine Geschäft der 13 Gegenseitigkeitsvereine hat einen Verlust von $791 \mathscr{H}$ und einen Gewinn von $198295 \mathscr{M}$ gebracht.

Dns

Ergebuls

der Ver-

nogens-

verwaltung

Das Frgebnis der Vermögensverwaltung wird von den Unternelımungen in der Regel nicht nach den einzelnen Geschäftszweigen getrennt; nur der Lebensversicherung werden immer bestiminte Erträge überwiesen, weil das der Betrieb dieses Geschäfts erfordert. Es besteht keine Veranlassung, in dieser Statistik von dem Verfahren der Gesellschaften alozugehen. Soweit also nicht ein Teil des Ertrags einem bestimmten Zweige zugewiesen ist, fülıren wir das Ergebnis nur insgesamt auf, und zwar im allgemeinen bei derjenigen Gruppe, zu der das Hauptgeschäft der Unternehmung gehört.

In Tafel IV 34 findet sich bei 36 Aktiengesellschaften der Ertrag der gesamten Vermögensverwaltung, bei 5 Gesellschaften nur der nicht der Lebensversicherung zukommende 'Teil; der gesamte Ertrag ist für 4 Gesellschaften bei Gruppe II nachgewiesen. Bei den Feuerversicherungs-Gegenseitigkeitsvereinen ist hier überall der Ertrag der gesamten Vermögensverwaltung aufgefülrt.

Unter den Erträgen stehen überall die Zinsen im Vordergrund mit $16000901 \mathscr{N}$ bei den Aktiengesellschaften und $4164646 \mathscr{M}$ bei den Gegenseitigkeitsvereinen. Der reine Ertrag der gesamten Vermögensverwaltung beläuft sich dort auf $14336453 \mathscr{N}$ und hier auf $3716966 \mathscr{M}$.

Die Bilanz Eine Bilanz ist in dieser Statistik grundsätzlich nur für das Gesamtgeschäft einer jeden Gesellschaft gegeben und bei der Gruppe aufgeführt, zu der das Unternehmen seinem IIaupthetriebe nach - die Transportversicherung ausgeschlossen - gelıört. Dementsprechend sind die Bilanzen der Providentia, Frankfurter Versicherungs-Gesellschaft, und der Bayerischen Versicherungs-Bank bei Gruppe I und der Allianz, des Nordstern Unfall- und Haftpflicht-Versicherungs-Aktien-Gesellschaft, der Frankfurter Allgemeinen Versicherungs-Aktien-Gesellschaft und der Manmheimer Versicherungsgesellschaft bei Gruppe II behandelt, so daß in Tafel IV 38 die Bilanzen nur für 34 deutsche Aktiengesellschaften und 24 Gegenseitigkeitsverrine darzustellen sind. Faßt man diese zusammen, so schließt die Bllanz für die Alitiengesellschaften mit $773159931 \mathscr{A}$ und für die Gegenseitigkeitsvereine mit $120677463 \mathscr{N}$ ab. Im übrigen mögen folgende Zahlen hier zusammengestellt werden: 


\begin{abstract}
Vermógenswerto
Verpflichtungen der Aktionäre oder Garanten

Forderungen... . . . . . ........

Kaputalanlagen . ...... . , . . .

Kassenbestand
\end{abstract}

$\cdots$

Verbindlichkeiten

Aktıen- oder Garantiehapital ........

Gesetzlıche Rucklage ... . . . . ..

Sonderrüchlagen . $\ldots \ldots \ldots \ldots \ldots$

Guthaben von Versicherungsunternehmungen... .

Pràmuenüberträge $\ldots \ldots \ldots \ldots \ldots \ldots$.

Schadenruklage ... . . ........ .
34 Alitiengesellschaften

$182999100 \mathscr{M}$

140979264

445940595 .

651943
24 Gegenscitigkeitsvereme

$8 \mathrm{I} 6000 \mathscr{M}$

$12019542 "$

106244630 " 278041

Die Kapitalanlagen ('Tafel IV 39) verteilen sich auf folgende Wertarten:

Grundbesitz . .

Hypothehen und Grundschulden.........

Darlehen an öffentliche Korperschaften.

Darlehen auf Wertpapiere........ . . ...

Wertpapiere.... ........... ...

Darlehen auf Versicherungen ... . ... . .

Wechsel .....

Sonstige Anlagen
$247203580 \mathscr{M}$
39059282 .
74405882 "
63067549 .
$19935^{2} 946$,
83024743 "

$2170631 \mathscr{A t}$
$34470491 "$
$7657514 "$
$953939 "$
$22474652 "$
$3711610 "$

bei 34 Ahtiengesellschaften $33195040 \mathscr{N}$ oder $7,4 \mathrm{~V} \mathrm{H}$. 192862 II7 " " 43,3 * $1100000 \cdot 00,2$ " $4514459 " . \quad 1,0$. 201423545 * " 45,2 " 6151797 " 1,4 " 4838879 . " 1,1 . $185475^{8}$ " . 0,4 " bel 24 Gegensertugheitsveremen $2828406 \mathscr{N}$ oder 2,7 v H 36080844 " " 33,9 " 10526 III " " 9.9 " - " - - " $55352417 "$ " 52, I " - " - " $406600 ", 04$ " 1050252 " " 1,0 "

Vergleicht man die Kapitalanlagen der in der Bilanz aufgeführten Unternelımungen am Ende des Berichtsjalırs mit den entsprechenden Anlagen am Ende des Vorjahrs, so findet man eine Zunalıme um $1766719 \mathscr{H}$ bei den Darlehen auf Wertpapiere, um $52837699 \mathscr{M}$ beim Buchwerte der Wertpapiere (um rund 59 Millionen beim Nennwert) und um $3877625 \mathscr{M}$ bei dem Bestand an Wechseln. Zurückgegangen sind der Grundbesitz um $288420 \mathscr{M}$, die Hypotheken und Grundschulden um $13069491 \mathscr{N}$, die Darlehen an öffentliche Körperschaften um $46081 \mathscr{A}$, die Darlehen auf Versicherungen um $4202 \bar{i} \mathscr{N}$ und die sonstigen Anlagen um $1354368 \mathscr{M}$.

Die Bilanz weist bei 1 Gegenseitigkeitsverein und bei 1 Aktıengesellschaft weder einen Gewinn noch einen Verlust nach. Alle anderen Unternehmungen haben günstig abgeschlossen. Der bilanzmäßige Gewinn beträgt für die Altiengesellschaften $32601423 \mathscr{H}$ und für die Gegenseitigkeitsvereine $28967072 \mathscr{N}$; er ist nach Tafel IV 40 wie folgt verteilt worden:

\begin{tabular}{|c|c|c|c|c|c|c|c|c|c|}
\hline & \multicolumn{4}{|c|}{33 Aktiengesellschaften } & \multicolumn{5}{|c|}{23 Gegenseitigkeitsvereıne } \\
\hline an die gesetzliche Rucklage & 813769 & $\mathscr{M}$ oder & $2,5 \mathrm{v}$ & $\mathrm{H}$ & I $126 \quad 5_{5}^{2}$ & $\mathscr{M}$ & ode & 3,9 & v. H. \\
\hline an sonstige Ruchlagen. & 5607649 & $"$ & 17,2 & - & 2383312 & $"$ & $"$ & 8,2 & $n$ \\
\hline an den Beanitenwohlfahrtsstock & 978825 & $"$ & 3,0 & , & 688105 & $"$ & " & 2,4 & $"$ \\
\hline an die Aktıonäre (Garanten) . & 16034393 & . & 49,2 & " & $295^{\circ}$ & $n$ & . & & $"$ \\
\hline$\ldots \ldots \ldots \ldots \ldots$ & 2726862 & $n$ & 8,4 & n & 29522 & " & $"$ & 0,1 & $n$ \\
\hline an die Versicherten.$\ldots \ldots \ldots$ & - & $n$ & - & " & 227 I93 & 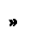 & $"$ & 83,6 & - \\
\hline anderweit $\ldots \ldots \ldots, \ldots, \ldots, \ldots \ldots$ & 1895822 & . & 5,8 & $n$ & 58893 & n & $n$ & 0,2 & n \\
\hline Vortrag auf neue Rechnung ........ & $4544 \quad 103$ & $n$ & I 3.9 & $n$ & 451045 & $n$ & $\bowtie$ & $\mathrm{I}, 6$ & $n$ \\
\hline
\end{tabular}

Für die ausländischen Gesellschaften sind die Bilanz der Assicurazioni Generali bereits bei der Lebensversicherung in Tafel I 47, die Bilanzen der Ersten Österreichischen Allgemeinen Unfall-Versicherungs-Gesellschaft, der Schweizerischen National-Versicherungs-Gesellschaft, der Schweizerischen Unfallversicherungs-Gesellschaft und der Zürich bei der Unfall- und Haftpflichtversicherung in Tafel II 14 und die Bilanz der 
Union Suisse beı der Glasversıcherung in Tafel V 13 gegeben; die Bilanzen der übrigen 9 Unternehmungen finden sich in Tafel IV 42. Im Anschlusse daran sind in Tafel IV 43 die Kapitalanlagen und die Forderungen erläutert. Wegen des großen Interesses, das der Art der Kapitalanlagen zukommt, sind in der folgenden Ubersicht die Gesellschaften nach Heimatländern zusammengefaßt; zum Vergleiche sind die Verhältniszahlen für die deutschen Unternehmungen wiederholt.

Von je $1000 \mathscr{M}$ Kapitalanlagen entfielen auf:

\begin{tabular}{|c|c|c|c|c|c|c|c|c|c|}
\hline ber & $\begin{array}{c}\text { Grund- } \\
\text { besitz }\end{array}$ & $\begin{array}{l}\text { Hypo- } \\
\text { theken } \\
\text { und } \\
\text { Grund- } \\
\text { schulden }\end{array}$ & $\begin{array}{l}\text { Darlehen } \\
\text { an offent- } \\
\text { liche } \\
\text { Korper- } \\
\text { schaften }\end{array}$ & $\begin{array}{c}\text { Darlehen } \\
\text { auf } \\
\text { Wert- } \\
\text { papiere }\end{array}$ & $\begin{array}{l}\text { Wert- } \\
\text { papiere }\end{array}$ & $\begin{array}{l}\text { Darlehen } \\
\text { auf Ver- } \\
\text { siche- } \\
\text { rungen }\end{array}$ & $\begin{array}{c}\text { Wech- } \\
\text { sel }\end{array}$ & $\begin{array}{l}\text { Son- } \\
\text { stige } \\
\text { An- } \\
\text { lagen }\end{array}$ & $\begin{array}{c}\text { Gesamtbetrag } \\
\text { der } \\
\text { Kapitalanlagen }\end{array}$ \\
\hline 1 belgischen Gesellschaft. & 59 & - & - & 112 & 829 & - & - & - & $4329059 \mathscr{N}$ \\
\hline 1 dämschen Gesellschaft & 228 & 368 & - & - & 404 & - & - & - & $357585^{8}$ n \\
\hline 2 miederlandischen Gesellschaften & 158 & 26 & 一 & 182 & 609 & - & - & 25 & 13311923 \\
\hline 1 osterreıchıschen Gesellschaft. & - & $3 I$ & I 5 & - & 927 & 12 & 15 & - & 24650877 . \\
\hline 2 schwedischen Gesellschaften & 40 & 469 & 45 & 44 & 332 & 70 & - & - & 98934690 \\
\hline $\begin{array}{c}2 \text { schweızerischen Gesellschaften } \\
\text { dagegen beı }\end{array}$ & 117 & 455 & - & - & 388 & - & 40 & - & 20096606 . \\
\hline 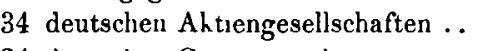 & 74 & 433 & 2 & 10 & $45^{2}$ & It & I I & 4 & 445940595 \\
\hline 4 deutschen Gegenseitıgkeitsvereınen & 27 & 339 & 99 & - & 521 & - & 4 & 10 & $106244630=$ \\
\hline
\end{tabular}

Von den 12 kleinen Vereinen, die in den Tafeln nicht behandelt sind, muß einer wegen unvollständiger Angaben hier ganz ausscheiden, bei den übrigen kommen insgesamt 47431 Versicherungen mit rund 402,81 Millionen Mark in Frage; der durchschnittliche Betrag einer Versicherung stellt sich danach auf $8493 \mathscr{M}$. Die gesamten Einnahmen haben im Jahre 19 $6372928 \mathscr{M}$ betragen; davon kommen $182730 \mathscr{H}$ oder 49,0 v. H. auf Beiträge, $55558 \mathscr{H}$ oder 14,9 v. H. auf Nebenleistungen der Versicherten und $53764 \mathscr{M}$ oder 14,4 v. H. auf Kapitalerträge; von den gesamten Ausgaben in Höhe von $308788 \mathscr{M}$ kommen $165364 \mathscr{M}$ oder 53,5 v. H. auf Entschädigungen und $79872 \mathscr{M}$ oder 25,9 v. H. auf Verwaltungskosten. Als Vermögen am Schlusse des Berichtsjahrs haben die 12 Vereine zusammen $1374698 \mathscr{M}$ nachgewiesen.

Diese Zahlen lassen jedoch kein Urteil über die Bedeutung der kleinen Vereine im Deutschen Reiche zu. Die Zahl der überhaupt vorhandenen kleinen Vereine ist viel größer; auch werden sich unter der Aufsicht der Landesbehörden gewiß mehrere finden, welche die unter Reichsaufsicht stehenden kleinen Vereine an Bedeutung übertreffen.

\section{Gruppe V, Sonstige Versicherungszweige Glasversicherung}

Auf dem Gehiete der Glasversicherung berichtet die Statistik für das Jahr 19 6 uber 21 deutsche Aktiengesellschaften, 2 deutsche Gegenseitigkeitsvereine und 3 ausländische Gesellschaften. 4 Aktiengesellschaften und 1 Gegenseitigkeitsverein beschäftigten sich ausschließlich mit diesem Versicherungszweige; für die übrigen Unternehmungen bildet die Glasversicherung nur einen Nebenbetrieb.

Die National Provincial Plate Glass \& General Ins. Co. Ltd. in London hat nach Ausbruch des Krieges mit einer deutschen Gesellschaft einen Haftungs- und Ubergangsvertrag abgeschlossen und ist deshalb auch aus dieser Statistik fortgelassen. 
Uber den Umfang des Geschäfts der deutschen Unternehmungen in der Glasver- Der Unfang sicherung soll Tafel V 1 unterrichten. Da die Versicherungssumme bei einer Gesellschaft nicht vollständig angegeben werden kann, so empfiehlt sich hier die Beschränkung auf diejenigen Gesellschaften, welche für die Jahre 1915 und 1916 alle Angaben haben des Geseháfts der deutsehen machen können. Bei der Besprechung der Beiträge und der Schäden im Anschluß an Tafel V 4 wird Gelegenheit sein, auf den Geschäftsumfang aller Gesellschaften im Jahre I 9 I 6 zurückzukommen.

An selbst abgeschlossenen Versicherungen bestanden einschließlich der in Rückdeckung gegebenen Beträge:

\begin{tabular}{|c|c|c|c|c|c|c|c|c|c|}
\hline \multirow{3}{*}{$\begin{array}{c}\text { bei } 20 \text { Aktiengesellschaften . } \\
\text { • } 2 \text { Gegenseitigkeitsr ereinen }\end{array}$} & \multicolumn{5}{|c|}{ Ende 1915} & \multicolumn{4}{|c|}{ Ende I9I 6} \\
\hline & 625955 & Vers. & uber & 351217340 & $\mathscr{H}$ & 614983 & Vers & n̈ber & 356488530 \\
\hline & 22805 & $n$ & $n$ & I I 826544 & . & 22161 & $n$ & $n$ & 12022234 \\
\hline
\end{tabular}

Die durchschnittlich auf eine selbst abgeschlossene Versicherung entfallenden Beträge waren in Mark:

\begin{tabular}{|c|c|c|c|c|c|c|}
\hline Ende des Jahres & & 1912 & 1913 & 1914 & 1915 & 1916 \\
\hline Ahtiengesellschaften, Gesamtgeschaft . & . & 562 & $5^{62}$ & 562 & 561 & $5^{80}$ \\
\hline inlandisches Geschäft .. $\quad \ldots$ & . & 555 & 564 & 561 & 559 & 577 \\
\hline ausland:sches Geschaft . .. & ... & 639 & 547 & 576 & 584 & 605 \\
\hline Gegenseitıgheirsvereine, Gesamtgeschäft .. & . .. & 520 & $5^{20}$ & 518 & 5 I 9 & 542 \\
\hline inländisches Geschäft . .... & . & 525 & 524 & 521 & 522 & $54 \mathrm{I}$ \\
\hline auslandısches Geschäft .... & & 436 & 447 & 458 & 459 & 567 \\
\hline Insgesant & t. & 560 & 557 & 561 & 560 & $57^{8}$ \\
\hline
\end{tabular}

Außerdem haben die Aktiengesellschaften noch Versicherungen in IIöhe von $68673 \mathscr{M}$ am Schlusse des Jahres 1915 und in IIöhe von $913159 \mathscr{H}$ am Schlusse von 1916 als in Rückdeckung übernommen nachgewiesen. Der Anteil des mittelbaren Geschäfts am Gesamtgeschäft ist danach ganz unbedeutend gewesen; er stellte sich bei den Aktiengesellschaften im Jahre 1915 auf 0,02 und im Jahre 1916 auf 0,26 v. IH.

Von den selbst abgeschlossenenVersicherungen derjenigen Unternelimungen, bei denen die Zahl der Versicherungen und die Versicherungssummen angegeben sind, kommen:

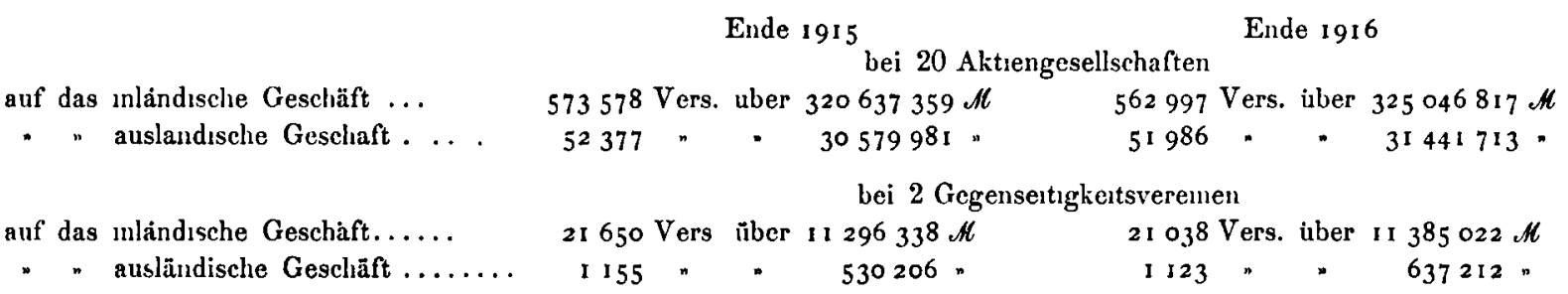

Der Umfang des deutschen Geschäfts der hier eingestellten ausländischen Gesellschaften ist nur ganz unbedeutend (Tafel V 3). Es waren Fnde I915 1880 Versicherungen über 837 (\$20 $\mathscr{N}$ und Ende I 9 I 62054 Versicherungen über $10003 \overline{4} 4 \mathscr{M}$ in Kraft. Der durchschnittlich auf eine Versicherung kommende Betrag belief sich am Ende des Vorjahrs auf $446 \mathscr{M}$, am Ende des Berichtsjahrs auf $48 \tau \mathscr{T}$. Das Geschäft ist von allen Gesellschaften nur unmittelbar betrieben worden.

Die Einnahme an Rohbeiträgen im Geschäftsjahr und die im Geschäftsjahr entstandenen Schäden sind für die deutschen Unternehmungen in Tafel V 4 und für das deutsche Geschäft der ausländischen Gesellschaften in Tafel V 5 zusammengestellt.

Dic Rohbestrage und Schaden 
In der Glasversicherung haben im Jahre 1916 die 21 deutschen Aktiengesellschaften $10012644 \mathscr{N}$ und 2 Gegenseitigkeitsvereine $325564 \mathscr{M}$, alle Unternehmungen zusammen also $10338208 \mathscr{H}$ an Beiträgen eingenommen; den Aktiengesellschaften sind Schäden im Betrage von $6845967 \mathscr{M}$, den Gegenseitigkeitsvereinen solche im Betrage von $184875 \mathscr{M}$, zusammen im Betrage von $7030842 \mathscr{M}$ entstanden.

Der durchschnittliche Betrag eines Schadens stellt sich für die Aktiengesellschaften im inländischen Betriebe beim unmittelbaren Geschäft auf $70 \mathscr{M}$, im ausländischen Betrieb auf $30 \mathscr{M}$, für die deutschen Gegenseitigkeitsvereine im Inland auf $65 \mathscr{N}$, im Ausland auf $56 \mathscr{M}$.

Aus dem deutschen Glasversicherungsgeschäfte (Tafel V 5) haben die 3 ausländischen Gesellschaften im Jahre I 9 I 6 insgesamt $23917 \mathscr{M}$ Rohbeiträge eingenommen; für Schäden hatten sie $11719 \mathscr{M}$, durchschnittlich für einen Schadenfall $84 \mathscr{M}$ bereitzustellen.

Die

Betriebs-

Einnabmen und

-Ausgaben

und der

Betriebs-

Gew In!

des

Berichts-

jahrs

Die Ergebnisse des Geschäftsbetriebs sind bei der Glasversicherung, die von den Unternehmungen meist nur als Nebenbetrieb behandelt wird, in derselben Weise dárgestellt wie bei der Feuerversicherung (S. 66*). Es ist in Tafel V 6 der Gewinn des Berichtsjahrs allein aus dem Betriebe der Glasversicherung ermittelt, indem die Betriebs-Einnahmen den Betriebs-Ausgaben nebst Verwaltungskosten und Steuern gegenübergestellt sind. Der Gesamtgewinn der Unternehmungen ist in der Bilanz ausgewiesen (Tafel V 9)

Die Betriebs-Finnahmen sind hier wie bei Gruppe IV die Beiträge für eigene Rechnung, die Nebenleistungen der Versicherten, die Ersparnis aus der Schadenrücklage des Vorjahrs, die Abnahme der Prämienüberträge und außerdem der Erlös aus Bruchglas; als Betriebs-Ausgaben wurden die Schäden des Geschäftsjahrs (nach Abzug des Anteils der Rückversicherer und der Schadenerstattungen), der Zuschuß zur Schadenrücklage des Vorjahrs und die Zunahme der Prämienüberträge zusammengefaßt.

Bei den deutschen Unternehmungen betrug in der Glasversicherung:

die Summe der Betriebs-Einnahnen "Betrielss-Ausgaben

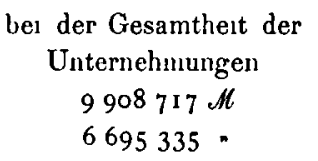

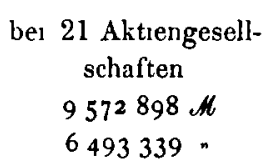

bei 2 Gegenseitıkkeitsveremen $335819 \mathscr{A l}$ 201996 .

Von je $1000 \mathscr{M}$ der Betriebs-Einnahmen kamen:

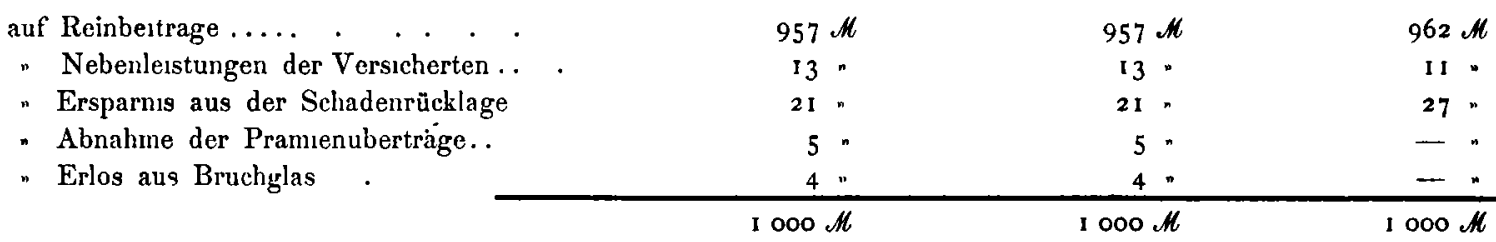

Von je $1000 \mathscr{M}$ der Betriebs-Ausgaben kamen:

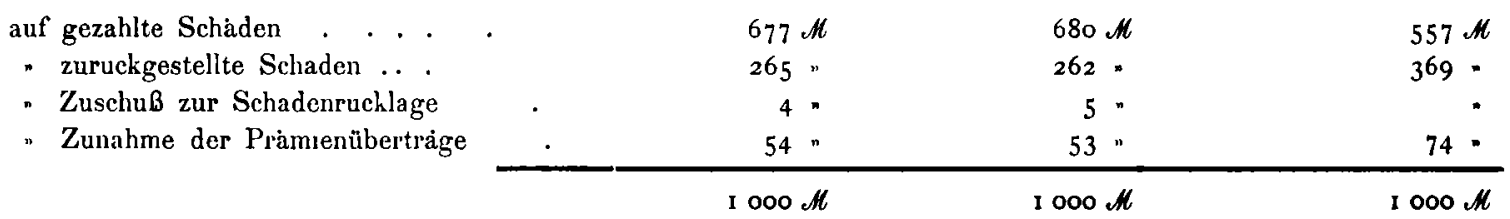


Von den im Geschäftsjahr entstandenen Schadensummen wurden durchschnittlich bei den Aktiengesellschaften 72,2 v. H., bei den Gegenseitigkeitsvereinen 60,1 v. I. und bei allen Anstalten zusammen 71,9 v. H. noch im Geschäftsjahre gezahlt; der Rest wurde der Schadenrückläge überwiesen.

An Verwaltungskosten und Steuern haben für die Glasversicherung die 21 Aktiengesellschaften $2954972 \mathscr{N}$ und die Gegenseitigkeitsvereine $113028 \mathscr{M}$ aufgewandt Aus dem reinen Versicherungsgeschäfte haben 10 Aktiengesellschaften einen Verlust von zusammen $281255 \mathscr{M}$ erlitten und die übsigen einen Gewinn ron zusammen $405842 \mathscr{M}$ erzielt; von den Gegenseitigkeitsvereinen hatte der eine einen Gewinn von $22444 \mathscr{M}$ und der andere einen Verlust von $1649 \mathscr{M}$.

Das Ergebnis der Vermögensverwaltung ist in Tafel V 7 nur für diejenigen deutschen Unternehmungen zusammengestellt, deren Hauptgeschäft die Glasversicherung ist; das sind 6 Aktiengesellschaften und ein Gegenseitigkeitsverein.

Unter den Erträgen stehen auch hier die Zinsen im Vordergrunde mit $488498 \mathscr{M}$ bei den Aktiengesellschaften und mit $43789 \mathscr{M}$ bei dem Gegenseitigkeitsverein Der reine Ertrag beläuft sich bei den Aktiengesellschaften auf $557795 \mathscr{M}$ und bei dem Gegenseitigkeitsverein auf $40552 \mathscr{M}$.

Die Bilanz der deutschen Unternehmungen ist in Tafel V 9 für dieselben $6 \mathrm{Ak}$ tiengesellschaften und einen Gegenseitigkeitsverein gegeben, die des andern Vereins befindet sich bei Gruppe IV. Sie schließt für Ende 1916 ab mit $21289184 \mathscr{A}$ bei den Aktiengesellschaften und mit $1092866 \mathscr{H}$ bei dem Gegenseitigkeitsverein. Im übrigen mögen hier folgende Zahlen zusammengestellt werden:

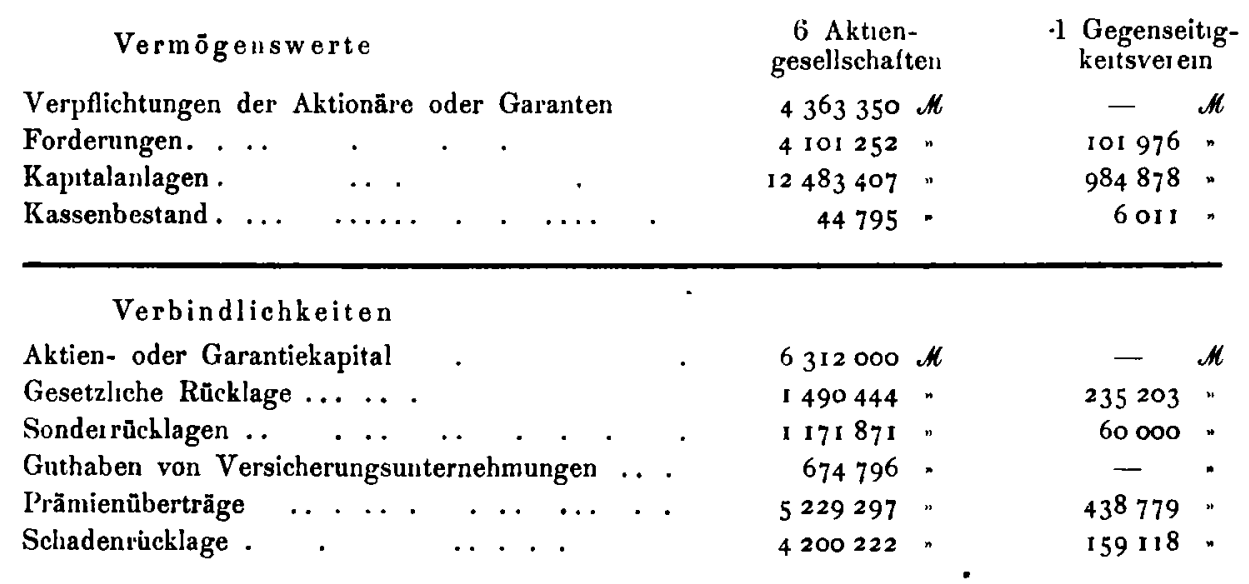

Die Kapitalanlagen (Tafel V 10) verteilen sich auf folgende Wertarten:

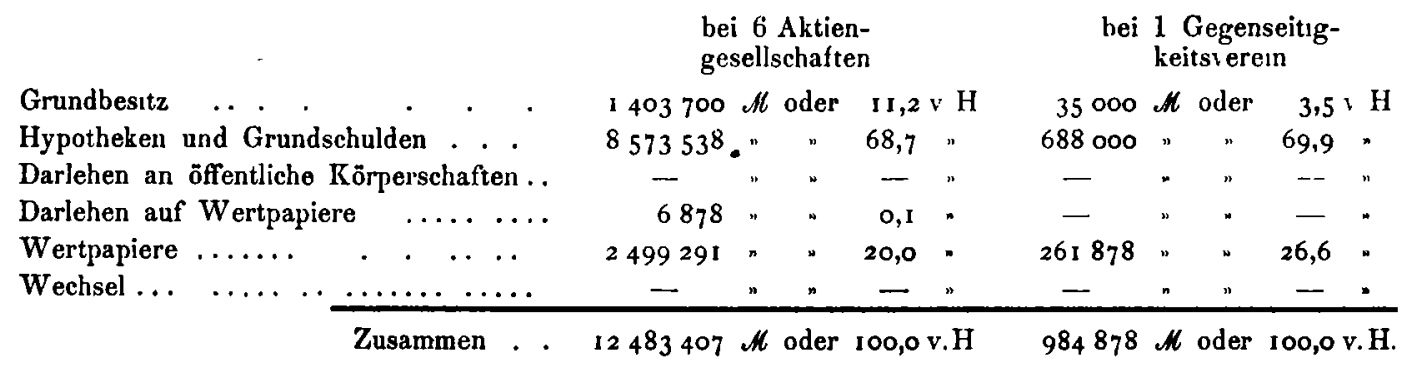


Die Kapitalanlagen sämtlicher 7 Unternehmungen haben Ende 1916 insgesamt $649908 \mathscr{M}$ mehr betragen als am Schlusse des Vorjahrs. Zugenommen haben der Hypothekenbestand um $178500 \mathscr{M}$ und die Wertpapiere um $494643 \mathscr{M}$; dagegen sind der Grundbesitz um $20300 \mathscr{M}$ und die Darlehen auf Wertpapiere um $2935 \mathscr{M}$ zurückgegangen.

Der bilanzmäßige Gewinn ist nach Tafel V 11 wie folgt verteilt worden:

\begin{tabular}{|c|c|c|c|c|c|c|c|c|c|}
\hline \multirow[b]{2}{*}{ an dıe gesetzlıche Rücklage .. } & \multirow[b]{2}{*}{.} & \multicolumn{4}{|c|}{$\begin{array}{l}\text { bei } 4 \text { Aktien- } \\
\text { gesellschaften }\end{array}$} & \multicolumn{4}{|c|}{ bei $\underset{\text { kettsver ein }}{1}$ Gegenseitig- } \\
\hline & & $17 \mathrm{I} 21$ & $\mathscr{N}$ oder & $1,9 \mathrm{v}$ & $\mathrm{H}$ & 1371 & $\mathscr{H}$ oder & $2,5 v$ & $\mathbf{H}$ \\
\hline an sonstige Ruchlagen . ... . & . $\ldots$ & 280400 & $n$ & 31,7 & . & - & $n \quad n$ & - & . \\
\hline an den Bcamtenwohlfahr tsstock & .. & - & . & - & " & - & . & - & " \\
\hline an die Aktionare (Garanten) & - & $35^{2} 170$ & $"$ & 39,9 & " & - & $"$ & - & $"$ \\
\hline für Tantiemen ... & . . . & I 18848 & $"$ & 13,4 & $"$ & 21939 & $"$. & 40,0 & " \\
\hline an die Versicherten & & - & n & - & $"$ & 31536 & 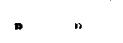 & 57,5 & $n$ \\
\hline anderweit . . & . . . & 47500 & $"$ & 5,4 & $"$ & - & $"$ & 一 & $"$ \\
\hline Vortrag auf neve Rechnnng &.$\quad$. & 67717 & $n$ & 77 & $n$ & - & $"$ & 一 & n \\
\hline
\end{tabular}

Die gesetzliche Rücklage steht in der diesjährigen Bilanz bei den Aktiengesellschaften um $36979 \mathscr{M}$ und bei dem Gegenseitigkeitsverein um $16838 \mathscr{M}$ höher als in der vorjälırigen Bilanz. Die Sonderrücklagen sind bei den Aktiengesellschaften um $299880 \mathscr{M}$ und bei dem Gegenseitigkeitsverein um $7000 \mathscr{A}$ gewachsen.

Für die ausländischen Gesellschaften ist die Bilanz der Assicurazioni Generali bei der Lebensversicherung in Tafel I 47 und die Bilanz der Schweizerischen NationalVersicherungs-Gesellschaft in Tafel II 14 gegeben; die Bilanz der andern Unternehmung findet sich in Tafel V 13. Im Anschlusse daran sind in Tafel V 14 die Kapitalanlagen und die Forderungen erläutert.

\section{Kautions- und Bürgschaftsversicherung}

Dieser Versicherungszweig wird nur nebenher betrieben. Auf das kleine Geschäft, das der Atlas in Ludwigshafen, die Willelma in Magdeburg und die Bayerische Versicherungs-Bank in München auf dem Gebiete der Kautionsversicherung erledigt haben, braucht hier nicht eingegangen zu werden (vgl. die Anmerkungen 1, 2 und 3 zu Tafel I 30 S. 92). Von ausländischen Gesellschaften waren zum Betriebe dieses Zweiges im Deutschen Reiche befugt die Schweizerische Unfallversicherungs-Alitiengesellschaft in Winterthur und die Zürich, Allgemeine Unfall- und Haftpflicht-Versicherungs-Aktiengesesellschaft in Zürich.

Über den Umfang des Geschäfts der hier behandelten 3 deutschen Aktiengesellschaften und seine Zusammensetzung aus unmittelbaren und mittelbaren Abschlüssen sowie über den Anteil des inländischen Geschäfts am Gesamtgeschäfte gibt Tafel V 15 Aufschluß. Danach liefen bei diesen Gesellschaften insgesamt an selbst abgeschlossenen Versicherungen einschließlich der in Rückdeckung gegebenen Beträge:

$$
\begin{aligned}
& \text { Ende 1915... . . } 2876 \text { Versicherungen uber } 59707200 \text { A } \\
& \text { " } 1916 \ldots 3372 \text { " } 3699879 \text {. }
\end{aligned}
$$

so daß die Zahl der Versicherungen um 496 und die versicherten Summen um $31291679 \mathscr{M}$ zugenommen haben. Außerdern hatten die Gesellschaften Ende i 9 I 6 noch $131425012 \mathscr{M}$ gegen $118602873 \mathscr{N}$ am Ende des Vorjahrs in Rückdeckung übernommen. 
Von dem gesamten übernommenen Betrage von $222423891 \mathscr{M}$ liefen $108238500 \mathscr{M}$ für eigene Rechnung.

Von den selbst abgeschlossenen Versicherungen kamen:

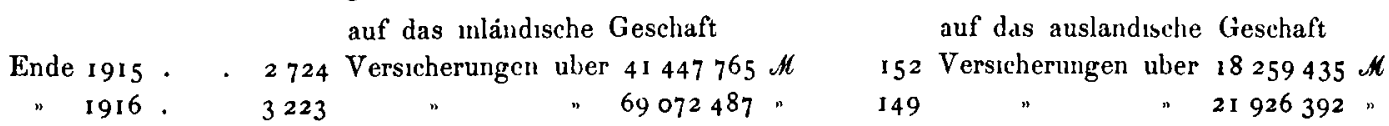

Die Rohbeitragseinnahme hat nach Tafel V 17 im Jahre I9I6 $1227421 \mathscr{M}$ betragen; davon kommen $1046163 \mathscr{M}$ auf das inländische und $181258 \mathscr{N}$ auf das ausländische Geschäft. Von den überhaupt entstandenen Schadensummen in Höhe von $740078 \mathscr{M}$ kommen $732117 \mathscr{M}$ auf das Inland und $7961 \mathscr{M}$ auf das Ausland.

Die Betriebs-Einnahmen betrugen $101386 i 6 \mathscr{M}$, während die Betriebs-Ausgaben $758806 \mathscr{M}$ ausmachten; an Verwaltungskosten und Steuern sind $185164 \mathscr{M}$ aufgewandt worden. Der Betriebsgewinn aus dem Kautions- und Bürgschaftsversicherungsgeschäfte hat sich zusammen auf $69896 \mathscr{N}$ belaufen.

Die beiden ausländischen Gesellschaften ('Tafel V 16) hatten Ende 1916 in Deutschland zusammen $8971710 \mathscr{N}$ versichert, gegenüber $10806540 \mathscr{N}$ am Ende des Vorjahrs; sie haben im Berichtsjahre $91843 \mathscr{N}$ an Beiträgen eingenommen und $51593 \mathscr{M}$ für Schäden vergütet.

\section{Kreditversicherung}

Die Kreditversicherung ist nur von geringer Bedeutung. Sie wird bei uns von 1 deutschen Aktiengesellschaft betrieben. Die Stuttgart-Berliner Versicherungs-Aktiengesellschaft in Stuttgart hat die Kreditversicherung fallen lassen. Von dem Kreditversicherungsgeschäfte einer ausländischen Gesellschaft kann hier wegen seiner Geringfügigkeit abgesehen werden; es genügt, kurz über das Geschäft der deutschen Unternelımung zu berichten.

Vom unmittelbaren Geschäfte waren in Kraft:

$$
\text { Ende } 1915 \ldots . .217 \text { Versicherungen uber } 3839000 \mathscr{A t}
$$

Es hat also die Zahl der Versicherungen um 44 ab- und die versicherte Summe um $115740 \mathscr{A}$ zugenommen. In Rückdeckung übernommen waren am Ende 1916 $796935 \mathscr{M}$.

Der weitaus größte Teil der überhaupt übernommenen Summen wurde in Rückdeckung weitergegeben, von 4,75 Millionen Mark blieben nur 1,53 Millionen für eigene Rechnung.

Das unmittelbare Geschäft stammt vollständig aus dem Deutschen Reiche.

Die Rohbeitragseinnahme hat nach Tafel V 17 im Jahre 1916 $362974 \mathscr{M}$ betragen; davon kommen $251852 \mathscr{M}$ auf das inländische und $111122 \mathscr{M}$ auf das ausländische Geschäft. Von den Schäden in einem Gesamtbetrage von $276491 \mathscr{M}$ entfallen $237168 \mathscr{N}$ auf den inländischen und $39323 \mathscr{N}$ auf den ausländischen Betrieb

Die Betriebs-Einnahmen betrugen $195195 \mathscr{M}$, wälırend die Betriebs-Ausgaben $106305 \mathscr{M}$ ausmachten; für Verwaltungskosten und Steuern sind $49436 \mathscr{M}$ aufgewandt worden. Die Gesellschaft hatte aus dem Kreditversicherungsgeschäft einen Gewinn von $39454 \mathscr{N}$ gehabt. 


\section{Maschinenversicherung}

Die Maschinenversicherung ist im Jahre 1916 bei uns von 2 deutschen Aktiengesellschaften und in geringem Umfange von 2 ausländischen Gesellschaften betrieben worden. Die Cölnische Unfall-Versicherungs-Aktien-Gesellschaft in Cöln a. Rhein hat die Maschinenversicherung fallen lassen. Neu hinzugekommen ist die Schweizerische National-Versicherungs-Gesellschaft in.Basel. Es genügt hier, nur den Geschäftsumfang der 2 deutschen Gesellschaften kurz zu erwähnen. Das Geschäft war fast ganz auf das Deutsche Reich beschränkt.

Nach 'Tafel V 15 waren an selbst abgeschlossenen Versicherungen in Kraft:

$\begin{array}{cccc}\text { Ende 1915 } & 2268 \text { Versicherungen uber } 393025640 \mathscr{M} \\ n 1916 & 2294 & " 50497025^{\circ}\end{array}$

Im Berichtsjahre hat also die Zahl der Versicherungen um 26 und die Versicherungssumme um $111944618 \mathscr{N}$ zugenommen.

Von diesen Versicherungen kamen:

auf das inlandische Geschaft

Ende 1915 . 2206 Versicherungen uber $374108770 \mathrm{M}$

" 1916 . $2233 \quad$ " 485449339 . auf das ausländısche Geschät 62 Versicherungen uber $18916870 \mathscr{M}$

61 " 19520919 .

In der Maschinenversicherung läuft nur ein verhältnismäßig kleiner 'Teil für eigene Rechnung: von 524,10 Millionen Mark 120,09 Millionen

Die Rohbeitragseinnahme hat nach Tafel V 17 im Jahre $19161073775 \mathscr{M}$ betragen; davon entfallen auf das inländische Geschäft $1002036 \mathscr{M}$, auf das ausländische Geschäft $71739 \mathscr{M}$. Für Schäden sind $854667 \mathscr{M}$ gezahlt worden; davon betreffen $794810 \mathscr{M}$ inländische und $59857 \mathscr{N}$ ausländische Schadenfälle.

Die Betriebs-Einnahmen haben $331490 \mathscr{M}$ betragen, während die Betriebs-Ausgaben $258008 \mathscr{M}$ ausmachten; für Verwaltungskosten und Steuern sind $80718 \mathscr{M}$ aufgewandt worden. Das Maschinenversicherungsgeschäft hat bei einer Gesellschaft einen Gewinn von $12021 \mathscr{M}$ und bei der anderen einen Verlust von $19257 \mathscr{M}$ ergeben.

\section{Baulastversicherung}

Die Baulastversicherung ist im Jahre 1916 nur unmittelbar und nur innerhalb des Deutschen Reichs von einem Gegenseitigkeitsverein betrieben worden.

Nach Tafel V 15 waren an selbst abgeschlossenen Versicherungen in Kraft:

$$
\begin{aligned}
& \text { Ende 1915. . } 239 \text { Versicherungen uber } 6477800 \text { At } \\
& \text { } 1916 . \ldots 240 \text { n } 24057300 \text { n }
\end{aligned}
$$

Es hat also im Berichtsjahre die Zahl der Versicherungen um 1 und die Versicherungssumme um $579500 \mathscr{M}$ zugenommen.

Von den übernommenen Versicherungen ist nichts in Rückdeckung gegeben worden.

Die Rohbeitragseinnahme hat nach Tafel V 17 im Jahre $191626526 \mathscr{M}$ betragen; für Schäden sind $22329 \mathscr{M}$ gezahlt worden.

Die Betriebs-Einnahmen haben $26526 \mathscr{M}$ betragen; demgegenüber stehen die Betriebs-Ausgaben mit $22696 \mathscr{M}$ und die Verwaltungskosten und Steuern mit $2284 \mathscr{M}$, so daß ein Gewinn von $1546 \mathscr{M}$ zu verzeichnen ist. 


\section{Wertgegenständeversicherung}

Diese Versicherungsart ist auch im Berichtsjahre nur von einer Aktiengesellschaft betrieben worden.

Es wurden nur unmittelbare Versicherungen (nur im Inlande) abgeschlossen. Das Geschäft umfaßte am Ende des Berichtsjahrs 421 Versicherungen über $6807190 \mathscr{M}$ gegenüber 429 Versicherungen über $6395312 \mathscr{N}$ am Ende des Vorjahrs. Für eigene Rechnung wurden nur 31 วิ3427 $\mathscr{N}$ belıalten. Die Beitragseinnahme belief sich auf $25607 \mathscr{M}$; für Schäden waren $8813 \mathscr{N}$ zu vergüten.

Die Betriebs-Einnahmen haben $15518 \mathscr{M}$ betragen, während die Betriebs-Ausgaben $5666 \mathscr{N}$ ausmachten; für Verwaltungskosten und Steuern sind $5955 \mathscr{A}$ aufgewandt worden. Das Wertgegenständeversicherungsgeschäft hat demnach einen Gewinn von $3897 \mathscr{M}$ ergeben.

\section{Veruntreuungsversicherung}

Die Veruntreuungsversicherung wurde auch im Berichtsjahre nur von einer Aktiengesellschaft und von dieser auch nur nebenher betrieben

Nach Tafel V 15 waren an selbst abgeschlossenen Versicherungen in Kraft:

$$
\begin{array}{ccccc}
\text { Ende } & 1915 & \ldots & 762 & \text { Versicherungen über } 18309090 \varkappa \\
n & 1916 & & 1009 & \text { " }
\end{array}
$$

In Rückdeckung übernommen waren am Ende $19162097520 \mathscr{N}$. Es hat also im Berichtsjahre die Zahl der Versicherungen um 247 und die Versicherungssumme um $7804550 \mathscr{N}$ zugenommen. Von dem gesamten übernommenen Betrage von $28211160 \mathscr{M}$ liefen $14884810 \mathscr{M}$ für eigene Rechnung

Die Rohbeitragseinnahme hat nach Tafel V 17 im Jahre I 9 I $6240895 \mathscr{M}$ betragen; für Sclıäden sind $47751 \mathscr{N}$ gezahlt worden.

Die Betriebs-Einnahmen betrugen $96409 \mathscr{N}$, während die Betriebs-Ausgaben $58974 \mathscr{N}$ ausmachten; an Verwaltungskosten und Steuern sind $41803 \mathscr{A}$ aufgewandt worden.

Das Veruntreuungsversicherungsgeschäft hat demnach einen Verlust von $4368 \mathscr{A}$ ergeben.

\section{Hypothekenversicherung}

Die Hypothekenversicherung ist im Berichtsjahre nur unmittelbar und nur innerhalb des Deutschen Reichs von einer Aktiengesellschaft betrieben worden.

Nach Tafel V 15 umfaßte das Geschäft am Ende des Berichtsjahrs 31 Versicherungen über $9082380 \mathscr{M}$ gegenüber 30 Versicherungen über $9070380 \mathscr{M}$ am Ende des Vorjahrs Für eigene Rechnung wurden nur 10 v. H. des versicherten Betrags behalten.

Die Rohbeitragseinnalume hat nach Tafel V 17 im Jahre 19i6 $50827 \mathscr{M}$ betragen, für Schäden sind $182492 \mathscr{A}$ gezalilt worden. Die Betriebs-Einnahmen haben $37052 \mathscr{M}$ betragen; demgegenüber stehen die Betriebs-Ausgaben mit $39465 \mathscr{M}$ und die Verwaltungskosten und Steuern mit $2408 \mathscr{M}$, so daß ein Verlust von $4821 \mathscr{N}$ $\mathrm{zu}$ verzeichnen ist. 


\section{Stellenlosenversicherung}

Die Stellenlosenversicherung wurde im Berichtsjahre unter Reichsaufsicht nur von zwei kaufmännischen Vereinigungen für ihre Mitglieder als Ergänzung ihrer Stellenvermittlung betrieben, und zwar olıne Rückversicherung

Das Geschäft war bisher nicht belangreich: Ende I9 I5 waren 136178 und Ende I 9 I6 143029 Vereinsmitglieder versichert. Die Betriebs-Einnahmen wiesen für das Berichtsjahr $84020 \mathscr{M}$ Beiträge auf. Als Stellenlosenunterstützung wurden $31661 \mathscr{M}$ ausgezalltt. Die Verwaltungskosten betrugen $44748 \mathscr{M}$.

\section{Automobilkaskoversicherung}

Die Automobilkaskoversicherung (gegen eigene Beschädigung der Wagen) ist im Berichtsjahre von der Rheinischen Feuerversicherungs-Aktiengesellschaft Cöln und vom Kronprinz, Versicherungs-Aktiengesellschaft Cöln nur innerhalb des Deutschen Reichs betrieben worden.

Nach Tafel V 15 waren an selbst abgeschlossenen Versicherungen in Kraft:

61 Versicherungen über $761860 \mathscr{M}$.

In Rückdeckung übernommen waren am Ende I 9 I $6190465 \mathscr{M}$.

Von dem gesamten übernommenen Betrage von $952325 \mathscr{M}$ liefen $380930 \mathscr{M}$ für eigene Rechnung.

Die Rolhbeitragseinnahme hat nach Tafel V 17 im Jahre I9I6 $3328 \mathscr{M}$ betragen; für Schäden sind $539 \mathscr{M}$ gezahlt worden.

Die Betriebs-Einnalımen haben $7502 \mathscr{N}$ betragen; demgegenüber stehen die Betriebs-Ausgaben mit $214 \mathscr{M}$ und die Verwaltungskosten und Steuern mit $408 \mathscr{M}$, so $\mathrm{da} ß$ ein Gewinn von $6880 \mathscr{N}$ zu verzeichnen ist.

\section{Fliegerschädenversicherung}

Die Fliegerschädenversicherung ist im Berichtsjahre von 3 Aktiengesellschaften, Allianz, Versicherungs-Aktien-Gesellschaft in Berlin, Union, Allgemeine VersicherungsAktien-Gesellschaft zu Berlin und Stuttgart-Berliner Versicherungs-Aktiengesellschaft in Stuttgart und von einem Gegenseitigkeitsvereine, Württembergische Feuerversicherung auf Gegenseitigkeit in Stuttgart betrieben worden.

Nach Tafel V 15 waren an selbst abgeschlossenen Versicherungen Ende I9I6 in Kraft:

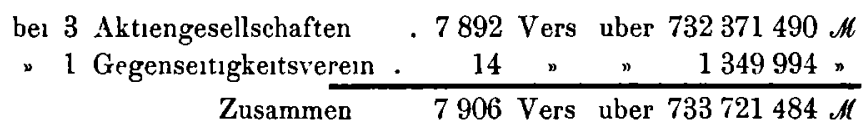

In Rückdeckung übernommen waren bei einer Aktiengesellschaft am Ende I9I6 $6156095 \mathscr{M}$.

Die Rohbeitragseinnahme hat nach Tafel V 17 im Jahre I9 6 bei den Aktiengesellschaften $1439212 \mathscr{M}$ und beim Gegenseitigkeitsvereine $6538 \mathscr{M}$ betragen; für Schäden sind bei den Aktiengesellschaften $1109661 \mathscr{M}$ gezahlt worden. 


\section{Kaskoversicherung}

Die Kaskoversicherung wurde im Jahre 1916 unter Reichsaufsicht von 37 deutschen Unternehmungen betrieben. Bei 2 Unternehmungen ruht die Versicherung während des Krieges. Die Kasko-Versicherungs-Gesellschaft "Rheinfahrt", Versicherungsverein auf Gegenseitigkeit, Homberg-Niederrhein, hat ilıren Sitz nach Duisburg verlegt. Wir beschränken demnach unsern Bericht für 1916 auf 35 Unternehmungen. Davon waren 32 Gegenseitigkeitsvereine und 3 eingetragene Genossenschaften.

Nach Tafel V 20 waren von diesen Unternehmungen versichert:

\begin{tabular}{|c|c|c|c|}
\hline Ende 1915 & 8247 & Fahrzeuge mit & 97767780 \\
\hline D 1916 & 7880 & " & 91885502 \\
\hline
\end{tabular}

Im Berichtsjahre hat die Zahl der versicherten Fahrzeuge um 367 und die Versicherungssumme um $5882278 \mathscr{N}$ abgenommen.

Die Summe der Einnalimen aller 35 Vereine hat im Jahre $19161289785 \mathscr{N}$ betragen, wälırend die Summe der Ausgaben $1340017 \mathscr{M}$ ausmachte. 18 Vereine haben einen Uberschuß von $65538 \mathscr{A}$ erzielt; 12 Vereine haben einen Verlust von $115770 \mathscr{M}$ erlitten (Tafel V 21).

Das Vermögen der Unternehmungen betrug Ende $19155545246 \mathscr{H}$ und Ende 1916 5614942 $\mathscr{N}$; es hat also um $69696 \mathscr{M}$ zugenommen und bestand Ende 1916 zu 13,0 v. H. in Sparkasseneinlagen, zu 64,0 v. H. in Wertpapieren, zu 9,2 v. H. in Hypotheken, zu 1,0 v. H. in barer Kasse und zu 12,8 v. H. in anderen Werten.

\section{Rückversicherung}

Von den deutschen privaten Versicherungsunternehmungen, welche die Rückversicherung in gesetzlich aufsichtspflıchtigen Versicherungszweigen zum Gegenstande haben, sind für das Jahr I 9 I 6 bei dieser Gruppe 40 behandelt, die in solchen Zweigen lediglich mittelbar arbeiten, und außerdem noch 5 , die in einigen $Z$ weigen unmittelbare und mittelbare, in andern nur mittelbare Geschäfte abschließen. Bei diesen letzteren Unternehmungen werden hier aber neben etwaiger Transportversicherung nur diejenigen aufsichtspflichtigen Zweige dargestellt, die nur mittelbar betrieben werden; dıe übrigen sind bereits in den vorhergehenden Gruppen behandelt. Zu den lediglich mittelbar arbeitenden Unternelımungen sind im Geschäftsjahre die Norddeutsche Versicherungsbank AktienGesellschaft in Berlin, die Transatlantische Güterversicherungs-Gesellschaft in Berlin, die Allgemeine Versicherungs-Gesellschaft für See-, Fluß- und Landtransport in Dresden, die Sächsische Rückversicherungs-Gesellschaft in Dresden und die Niederrheinische Güter-Assekuranz-Gesellschaft in Wesel hinzugekommen.

Die Rückversicherungs-Gesellschaft "Europa " in Frankfurt a. M. und die Frankona, Rück- und Mitversicherungs-Aktien-Gesellschaft in Frankfurt a. M., haben ihren Sitz nach Berlin verlegt.

Sümtliche Unternehmungen sind Aktiengesellschaften.

Ausländische Versicherungsunternehmungen der in Rede stehenden Art unterstehen nicht der Aufsicht des Amts und kommen daher hier nicht in Betracht. 

wird, sind die Gesellschaften nur bei der Lebensversicherung anzugeben in der Lage; bei den andern $Z$ weigen können nur die Beiträge und die Schäden herangezogen werden Unter diesen Umständen scheint eine besondere Behandlung der Versicherungssummen nicht angezeigt; es wird genügen, hier die Beiträge und Schäden nach Versicherungszweigen zusammenzustellen ('Tafel V 24):

\begin{tabular}{|c|c|c|c|c|c|}
\hline \multirow{3}{*}{ Versirherungszeig } & \multirow{3}{*}{$\begin{array}{c}\text { Zahl der } \\
\text { Gesell- } \\
\text { schaften }\end{array}$} & \multicolumn{3}{|c|}{ B e itrage } & \multirow{3}{*}{$\begin{array}{c}\text { S chäden } \\
\text { für } \\
\text { eigene Rechnung } \\
\mathscr{H}\end{array}$} \\
\hline & & \multirow{2}{*}{$\begin{array}{c}\text { Im ganzen } \\
\mathscr{M} \\
\end{array}$} & \multicolumn{2}{|c|}{ für eıgene Rechnung } & \\
\hline & & & $\mathscr{A H}$ & $v \mathrm{~T}$ & \\
\hline \multirow{8}{*}{$\begin{array}{l}\text { Lebensversicherung } \\
\text { Unfall- und Haftpflichtversıcherung } \\
\text { Transportı ersicherung . } \\
\text { Feuerversicherung } \\
\text { Hagelversicherung } \\
\text { Sonstige Zweige } \\
\\
\qquad \text { In Jahre } 1915 . \\
\text { Zusammen }\end{array}$} & 20 & 67457285 & 53276889 & 790 & $2042871 \mathrm{I}$ \\
\hline & 19 & 22875294 & 18678076 & 817 & $80_{5} 658$ \\
\hline & 34 & 184302670 & 79274740 & $43^{\circ}$ & 49956417 \\
\hline & $4 \mathrm{r}$ & 272054611 & $15456785^{8}$ & 568 & 80023548 \\
\hline & 4 & 6402331 & 6402331 & I 000 & 653226 I \\
\hline & 28 & 14052208 & 12120415 & 863 & 4271380 \\
\hline & 45 & 567144399 & 324320309 & 572 & 169217975 \\
\hline & 40 & 522641280 & 310348510 & 594 & $\cdot 171109425$ \\
\hline
\end{tabular}

Die Gewinn- und Verlustrechnung (Tafel V 23) bezieht sich auf den gesamten Geschäftsbetrieb der Unternehmungen; nur bei den bereits obenerwähnten finf Gesellschaften, die außer der Rückversicherung auch das unmittelbare Geschäft in aufsichtspflichtigen $Z$ weigen betreiben, sind hier neben der Transportversicherung nur diejenigen Zweige eingestellt, in denen sie lediglich mittelbar tätig sind. Wie sich das gesamte Geschäft aus den einzelnen Zweigen aufbaut, ist aus den bereits aus Tafel V 24 mitgeteilten Zahlen zu ersehen. Hier bleibt nur noch folgendes zu erwähnen.

Die Vermögenserträge beliefen sich insgesamt im Reinergebnis auf $21241250 \mathscr{M}$; einem Kursgewinne von $2087910 \mathscr{M}$ standen $461086^{\circ} 0 \mathscr{M}$ an Kursverlusten gegenüber. Von den Rücklagen haben nach Abzug der Abnahmen die Prämienreserven und Uberträge um $40557717 \mathscr{M}$, die Schadenrücklagen um $25155173 \mathscr{N}$ und die sonstigen Rücklagen um $516781 \mathscr{M}$ zugenommen. Die Verwaltungskosten und Steuern haben $92245569 \mathscr{M}$ betragen.

Von den 45 Gesellschaften haben zwei die Ausgaben mit den Einnahmen abgeglichen; die übrigen haben einen Gewinn von zusammen $14572104 \mathscr{M}$ und mit Einbeziehung des Gewinnvortrags aus dem Vorjahre von $21381524 \mathscr{M}$ erzielt.

Die Bilanzen der mehrfach erwähnten fünf Gesellschaften, welche aufsichtspflichtige Versicherungszweige auch unmittelbar betreiben, sind bereits bei Gruppe II in Tafel II 6 (Cölnische Unfall-Versicherungs-Aktien-Gesellschaft, Frankfurter Allgemeine Versicherungs-Aktien-Gesellschaft und Oberrheinische Versicherungs-Gesellschaft), oder bei Gruppe IV in Tafel IV 38 (Iduna und Stuttgart-Berliner Versicherungs-Aktiengesellschaft) nachgewiesen; es blieben hier also nur noch die Bilanzen für 40 Gesellschaften zusammenzustellen, was in Tafel V 25 geschehen ist. 
Aus dieser Tafel sind folgende Zahlen zu entmehmen:

40 Aktiengesellschaften

\begin{tabular}{|c|c|c|c|c|c|c|c|c|}
\hline \multicolumn{6}{|c|}{ Vermógenswerte } & \multicolumn{3}{|c|}{ Verbindlichkeiten } \\
\hline Verpflichtungen der & Aktionär & & e & 129725890 & $\mathscr{H}$ & Aktienkapıtal & 175674180 & $\mathscr{A}$ \\
\hline Forderungen.... & $\ldots \ldots$ & & $\cdots$ & 248762613 & " & Gesetzliche Rucklage & $409 ! 8173$ & $"$ \\
\hline Kapitalanlagen... & . & . & . . & 288060628 & n & Sonderrūcklagen .. & 36668801 & $"$ \\
\hline Kassenbestand & . $\quad$, & & . & 220193 & " & $\begin{array}{l}\text { Guthaben von Versicherungsunterne } \\
\text { mungen . } . \ldots \ldots \text {. . } \\
\text { Prämienreserven und Uberträge . } \\
\text { Schadenrucklage. }\end{array}$ & $\begin{array}{r}86388873 \\
184372848 \\
\text { I } 58 \Upsilon 27862\end{array}$ & " \\
\hline
\end{tabular}

Die Kapitalanlagen verteilen sich auf folgende Wertarten (Tafel V 26): $10757235 \mathscr{M}$ oder 3,7 v. H. Grundbesitz, $58715006 \mathscr{M}$ oder 20,4 v. H. Hypotheken, $210264285 \mathscr{M}$ oder 73,0 v. H. Wertpapiere, $4565053 \mathscr{M}$ oder 1,6 v. H. Wechsel, $1502465 \mathscr{M}$ oder 0,5 v. H. Darlehen an öffentliche Körperschaften, $328853 \mathscr{M}$ oder 0,1 v. H. Darlehen auf Wertpapiere und $1927731 \mathscr{M}$ oder 0,7 v. H. sonstige Anlagen.

Uber die Wertpapiere mag folgendes mitgeteilt werden: Dem Nennwerte nach handelt es sich bei den betrachteten 40 Gesellschaften um insgesamt $252980758 \mathscr{M}$, wovon $145391050 \mathscr{M}$ oder 57,5 v. H. auf inländische und $107589708 \mathscr{M}$ oder $42,5 \mathrm{v}$. H. auf ausländische Werte kommen. Die deutschen Anleihen bestehen aus $98504200 \mathscr{M}$ (38,9 v. H. aller Wertpapiere) Reichs- oder Staatsanleihen, $486900 \mathscr{M}(0,2$ v. H.) staatlich gedeckten Anleihen, $6266700 \mathscr{M}(2,5$ v. H.) anderen öffentlichen Anleihen, $10420000 \mathscr{M}(4,1$ v. H.) Pfandbriefen, $2730400 \mathscr{M}(1,1 \quad$ v. H.) Obligationen und $4279800 \mathscr{M}(1,7$ v. H.) Aktien von Banken, Eisenbahn- und sonstigen industriellen Unternehmungen und $22703050 \mathscr{M}(9,0 \mathrm{v}$. H.) Aktien von Versicherungsunternehmungen. Die ausländischen Anlagen bestehen aus $48218312 \mathscr{M}(19,1$ v. H. aller Wertpapiere $)$ Staats-, staatlich gedeckten und andern öffentlichen Anleihen, $31447817 \mathscr{M}(12,4 \mathrm{v} . \mathrm{H}$. sonstigen Schuldverschreibungen und $27923579 \mathscr{N}$ (11,0 v. H.) Aktien.

Als Prämienreserven und Uberträge sind, um die Ubereinstimmung mit den Bilanzen der andern Gruppen zu wahren, auch hier nur die in eigener Verwahrung und Verwaltung der betrachteten Unternehmungen befindlichen Beträge in die Bilanz eingestellt worden. Tafel V 27 zeigt, wie diese Beträge ermittelt wurden.

Der bilanzmäßige Gewinn dieser 40 Gesellschaften beträgt $20323046 \mathscr{M}$; er ist nach 'Tafel V 28 wie folgt verteilt worden: an die gesetzliche Rücklage $615705 \mathscr{M}$ oder 3,0 v. H., an die sonstigen Rücklagen $1528545 \mathscr{M}$ oder 7,5 v. H., an den Beamtenwohlfahrtsstock $272259 \mathscr{N}$ oder 1,4 v. H., an die Aktionäre $9322495 \mathscr{M}$ oder 45,9 v. H., als Tantiemen $1053814 \mathcal{M}$ oder 5,2 v. H., als Vortrag auf neue Rechnung $6854746 \mathscr{N}$ oder 33,7 v. H. und endlich anderweit $675482 \mathscr{N}$ oder 3,3 v. II. 



\section{Tafel}

zur

\section{Versicherungs-Statistik des Aufsichtsamts für 1916}




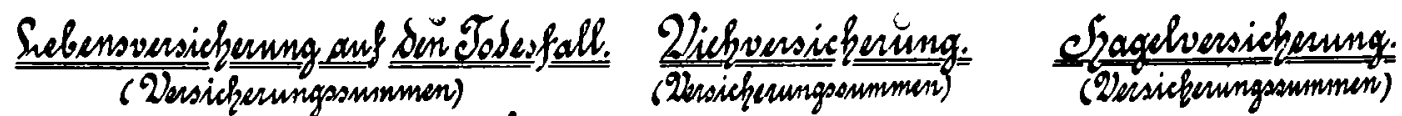

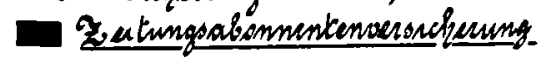

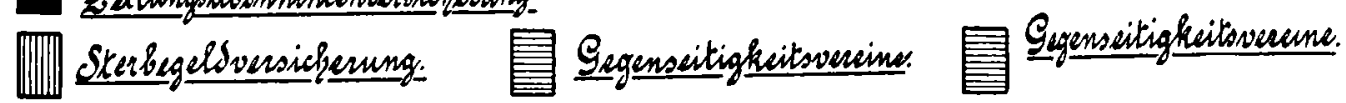

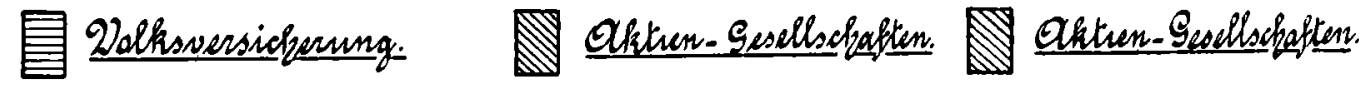
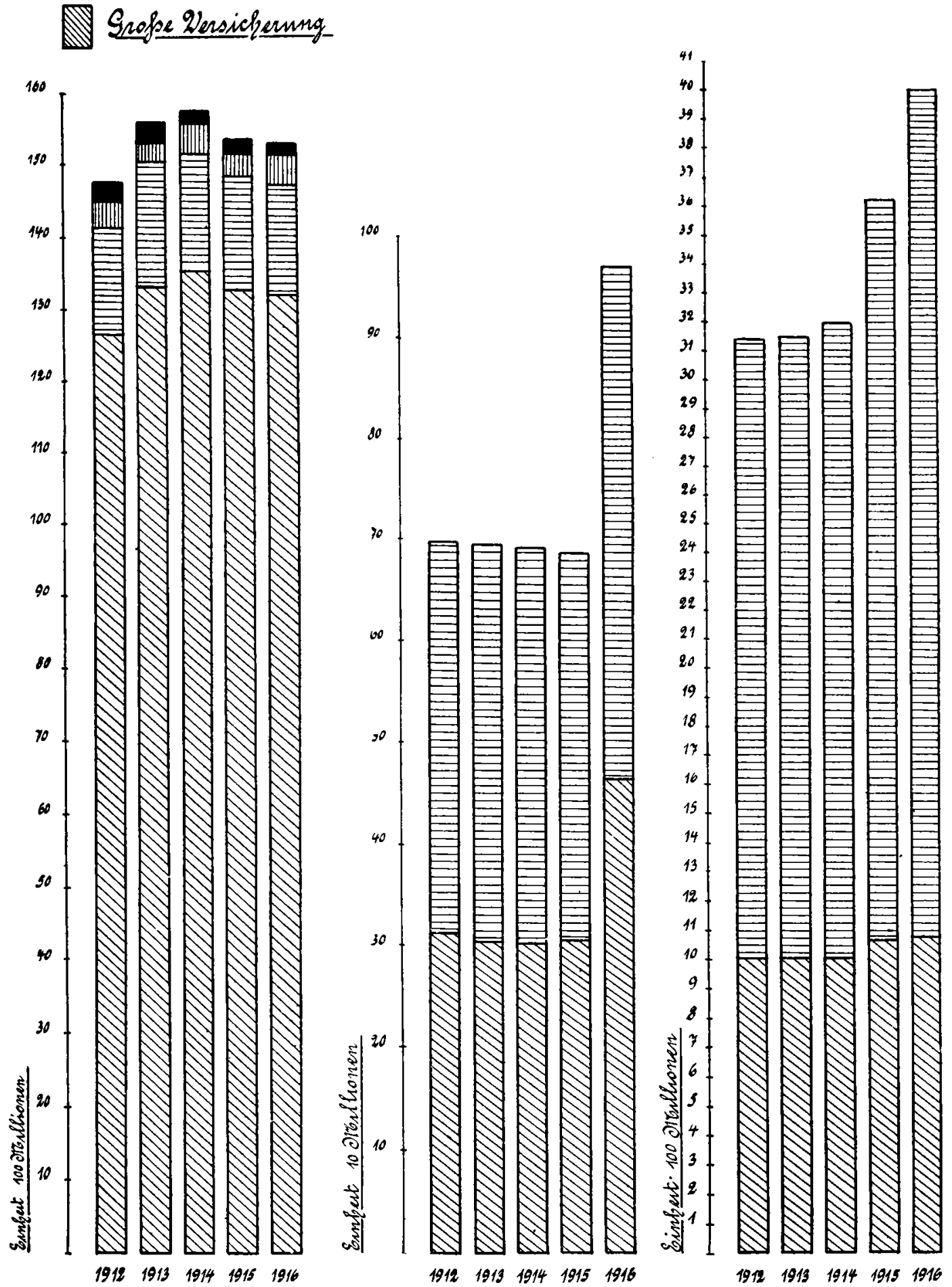

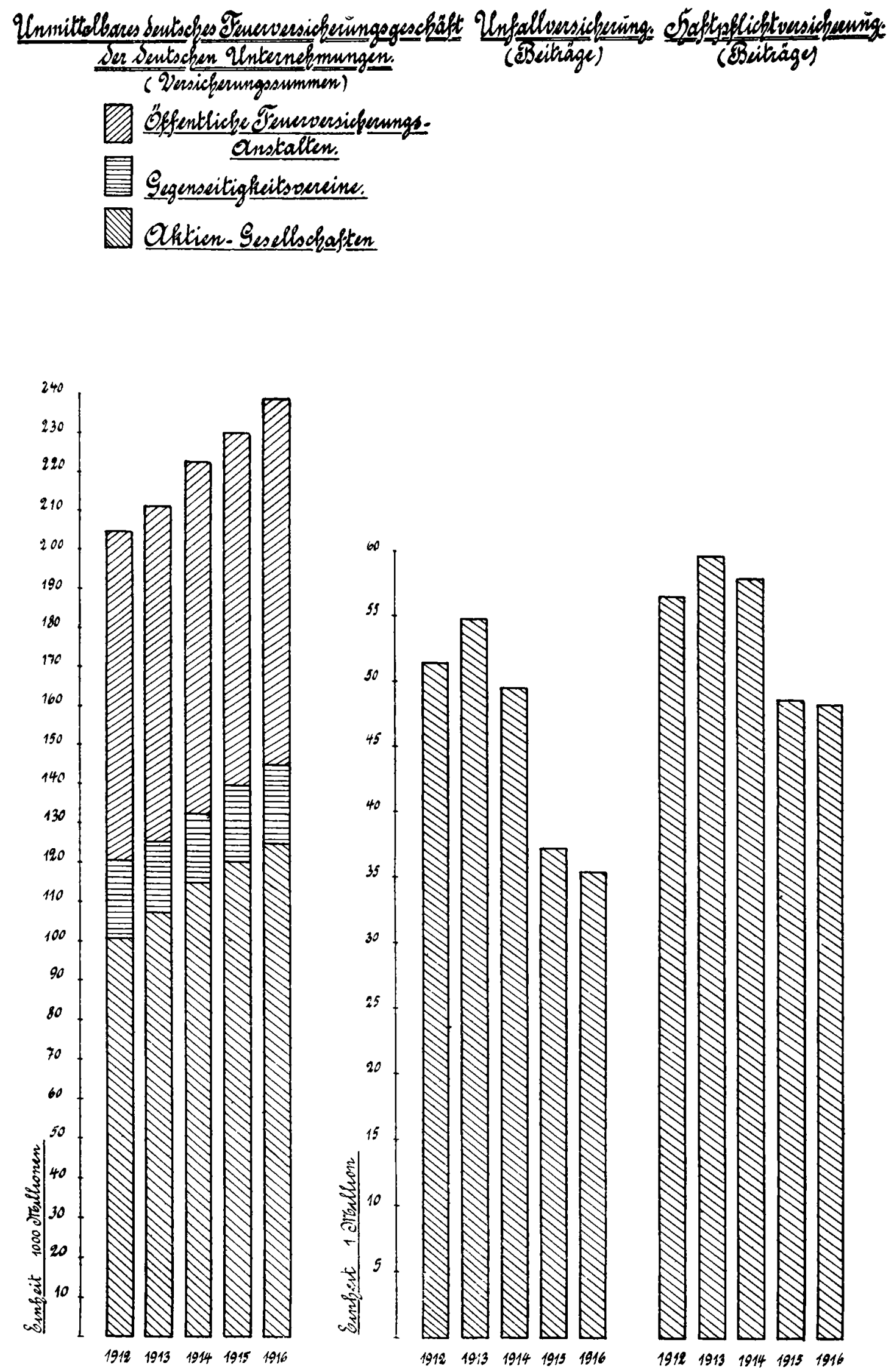
